\title{
A Survey of Factors Affecting Blunt Leading-Edge Separation for Swept and Semi-Slender Wings
}

\author{
James M. Luckring ${ }^{1}$ \\ NASA Langley Research Center, Hampton, VA, 23681
}

\begin{abstract}
A survey is presented of factors affecting blunt leading-edge separation for swept and semi-slender wings. This class of separation often results in the onset and progression of separation-induced vortical flow over a slender or semi-slender wing. The term semi-slender is used to distinguish wings with moderate sweeps and aspect ratios from the more traditional highly-swept, low-aspect-ratio slender wing. Emphasis is divided between a selection of results obtained through literature survey a section of results from some recent research projects primarily being coordinated through NATO's Research and Technology Organization (RTO). An aircraft context to these studies is included.
\end{abstract}

\section{Nomenclature}

\begin{tabular}{|c|c|c|c|}
\hline AR, A & aspect ratio & $\mathrm{R}_{\mathrm{mac}}$ & Reynolds number based on mac, $\mathrm{U}$ mac / $\mathrm{v}$ \\
\hline$C_{D}$ & drag coefficient & $\mathrm{R}_{\mathrm{r}}$ & Reynolds number based on r, U r / $v$ \\
\hline$C_{D, 0}$ & zero lift drag coefficient, uncambered wing & $\mathrm{r}$ & leading-edge radius, streamwise \\
\hline $\mathrm{C}_{\mathrm{L}}$ & lift coefficient & $\mathrm{S}_{\mathrm{ref}}$ & wing reference area \\
\hline $\mathrm{C}_{\mathrm{L}, \mathrm{o}}$ & lift coefficient at zero angle of attack & $\mathrm{s}$ & surface distance \\
\hline $\mathrm{C}_{\mathrm{L}, \max }$ & maximum lift coefficient & $\mathrm{t}$ & airfoil maximum thickness \\
\hline$\Delta \mathrm{C}_{\mathrm{L}}$ & $\mathrm{C}_{\mathrm{L}}-\mathrm{C}_{\mathrm{L}, \mathrm{o}}$ & $\mathrm{U}$ & free stream reference velocity \\
\hline $\mathrm{C}_{\mathrm{m}}$ & pitching moment coefficient & $u^{\prime}, v^{\prime}, w^{\prime}$ & fluctuating velocities \\
\hline $\mathrm{C}_{\mathrm{N}}$ & normal force coefficient & $\mathrm{x}, \mathrm{y}, \mathrm{z}$ & body-axis Cartesian coordinates \\
\hline $\mathrm{C}_{\mathrm{S}}$ & suction force coefficient & $\mathrm{x}_{\mathrm{v}}$ & $\begin{array}{l}\text { longitudinal distance to leading-edge vortex } \\
\text { origin }\end{array}$ \\
\hline $\mathrm{C}_{\mathrm{p}}$ & pressure coefficient & & \\
\hline $\mathrm{C}_{\mathrm{p}, \mathrm{rms}}$ & rms fluctuating pressure coefficient & $\alpha$ & angle of attack \\
\hline$\Delta \mathrm{C}_{\mathrm{p}}$ & lifting pressure coefficient & $\delta$ & boundary-layer thickness \\
\hline c & wing chord & $\phi$ & airfoil angular coordinate \\
\hline $\mathrm{d}$ & roughness diameter & $\eta$ & fraction semispan \\
\hline cref & reference chord & $\Lambda$ & wing sweep \\
\hline $\mathrm{C}_{1}$ & section lift coefficient & $\lambda$ & taper ratio \\
\hline $\mathrm{C}_{\mathrm{r}}$ & root chord & $\mu$ & viscosity \\
\hline K & boundary layer relaminarization parameter & $v$ & kinematic viscosity, $\mu / \rho$ \\
\hline $\mathrm{K}_{\mathrm{p}}$ & potential lift factor & $\rho$ & density \\
\hline $\mathrm{K}_{\mathrm{v}}$ & vortex lift factor & $\sigma$ & streamline coordinate \\
\hline$\ell$ & length & $\xi$ & characteristic length \\
\hline $\begin{array}{l}\mathrm{M} \\
\mathrm{mac}\end{array}$ & $\begin{array}{l}\text { Mach number } \\
\text { mean aerodynamic chord }\end{array}$ & $\zeta$ & Coordinate normal to surface \\
\hline $\mathrm{Q}$ & total velocity & Subscripts & \\
\hline Rbar & attachment line Reynolds number & a & attachment line \\
\hline $\mathrm{R}_{\mathrm{c}}$ & Reynolds number based on c, U c / v & e & edge of boundary layer \\
\hline
\end{tabular}

\footnotetext{
${ }^{1}$ Associate Chief Engineer for Aerosciences, Research Directorate, Mail Stop 499, AIAA Associate Fellow.
} 
AGARD

AVT

LTPT

NATO

NTF
Advisory Group for Aerospace Research and Development

Applied Vehicle Technology

Low Turbulence Pressure Tunnel

North Atlantic Treaty Organization

National Transonic Facility
RTO Research and Technology Organization

SACCON Stability and Control Configuration

TTCP The Technical Cooperation Program

UCAV Uninhabited Combat Air Vehicle

\section{Background}

mooth surface separation presents challenging fluid dynamic problems that can have significant aerodynamic consequences. These challenges have persisted for virtually the history of aircraft development. For many reasons, vehicle design practices are anchored in developing mission-relevant configurations that strive to achieve attached-flow aerodynamics for primary performance conditions. These practices have become quite refined and to a large degree are very successful in achieving the desired goals.

The onset and progression of smooth-surface separation nonetheless manifests in a number of ways. First, the onset of separation defines the boundaries of operation for any intended attached flow performance. The subsequent progression of separated flow can further establish the extent to which modified aerodynamic performance can be tolerated for the configuration. Next, multi-mission or multiple-performance-point requirements can result in separated flow having to become a condition which cannot be designed out of the vehicle with current technology. Under these circumstances, the separated flow must either be tolerated or, in some cases, exploited. Multidisciplinary constraints can further result in the occurrence of separated flow onset and progression within a vehicle's overall performance envelope. For example, low observable constraints can result in less wing twist and smaller leading-edge radii than might be desired from a purely aerodynamic perspective. Finally, lingering limitations in ground-based vehicle design practices can result in separated flow properties realized in flight that differ from those intended based upon the subscale development. Reynolds number scaling can be challenging for attached flow aerodynamics, and such scaling for the onset and progression of separated flow effects is certainly more so. Reliable prediction of the flow separation, either from experimental or computational means, is thus desirable regardless of whether the flow separation is to be avoided, tolerated, or perhaps even exploited.

The prediction of separation onset and progression continues to be problematical. Critical flow physics can occur at very small scales and in regions of high surface curvature, as in the case of the swept and blunt leading edge of slender wings, thus rendering experimental measurement very difficult. Many current computational technologies (e.g., Reynolds-Averaged-Navier-Stokes methods) incorporate turbulence models that are anchored in quasi steady attached flows, and it is unclear if such modeling can be expected to be applicable to the separated flow problem at hand.

Many categories of smooth surface separation remain of interest to configuration aerodynamics. These include, but are not limited to, blunt forebody separation, aft-body separation, swept wing trailing edge separation, transonic shock-induced wing separation, and blunt leading-edge separation for moderate-to-highly-swept slender wings. The intent for this paper is to provide a survey of information pertinent to smooth-surface (blunt) leading-edge separation for swept and slender wings. After a brief review of some wing properties for maneuvering aircraft, the work is organized into two primary thrusts.

The first thrust focuses on some fundamentals of blunt leading-edge separation obtained from a survey of the literature. It was a finding of this survey activity that the extensive information base on blunt-edge separation precludes a comprehensive treatment in the context of a conference article. Therefore, a subset of examples has been chosen to highlight in this paper that include both experimental and theoretical perspectives, and several applications to basic research configurations are included. The context of these studies provides an aid toward understanding the flows observed on more complex configurations.

The second thrust summarizes four recent research activities oriented toward more realistic configurations. These have been coordinated either through The Technical Cooperation Program (TTCP) or through the Research and Technology Organization (RTO, formerly AGARD). The first program was coordinated through the TTCP and was focused on aerodynamics of a representative UCAV configuration known as 1303. This activity included both experimental and numerical investigations and was completed several years ago. The remaining programs were coordinated through the RTO. The second program, AVT-113, recently completed research for blunt leading edge separation with both fundamental and aircraft oriented projects. The fundamental work was focused on a 65-degree delta wing while the aircraft work was focused on the F16-XL-1 aircraft. Both experimental and computational 
studies were included. The third program, AVT-161, is currently underway and is focused on blunt leading edge separation for a conceptual UCAV configuration known as SACCON. This configuration has a number of unique leading-edge separation characteristics in association with UCAV design considerations. The fourth program, AVT183, has only recently been initiated and is planning to draw upon current semi-slender aircraft interests (such as UCAVs) to formulate a fundamental investigation focused toward understanding some of the details of the blunt leading-edge separation onset phenomena. A relevant unit problem is currently envisioned for the focus of this activity.

\section{Aircraft Context}

The pursuit of high-speed flight with supersonic capability led to the aircraft configurations with what is broadly referred to as slender wings. Wave-drag considerations produce requirements for such wings to be positioned and sufficiently swept to lie behind a supersonic cruise Mach cone and to also be thin. Overall, this leads to wings with small leading-edge radii and with substantially lower aspect ratios than those used for efficient subsonic or transonic flight. For military aircraft with the added requirement for maneuver aerodynamics, the combination of low aspect ratio, high sweep, and small leading-edge radii further leads to the occurrence of separation-induced leading-edge vortex flows occurring at maneuver angles of attack. A review of the evolution of the slender wing in the context of vortex-lift research has been given by Polhamus ${ }^{1}$ [1986].

A purposely diverse sampling of military aircraft spanning many decades and design interests is presented in Figure 1. The F-106 entered service in 1959 as an upgraded version of the 1956 F-102 which, for the most part, represents the beginning

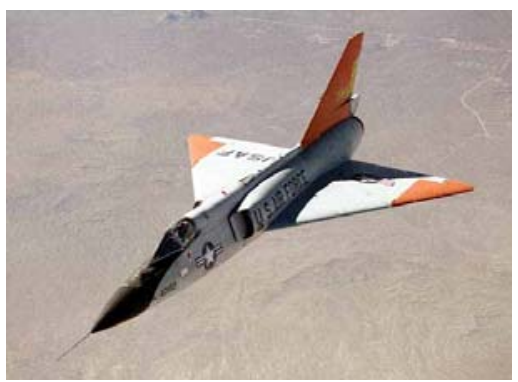

a) F-106

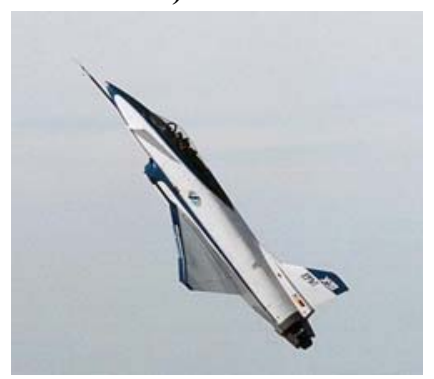

c) $X-31$

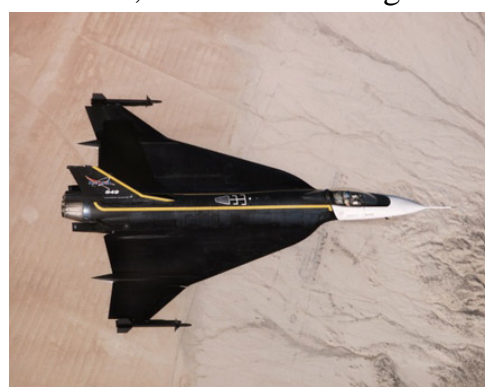

b) F-16XL

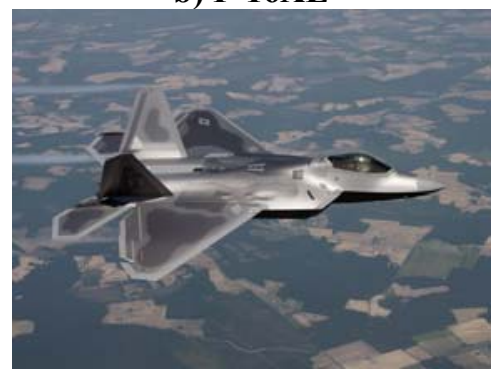

d) F-22

Figure 1. A sampling of maneuvering aircraft.

of maneuvering aircraft with supersonic capability due to the implementation of the area rule by Whitcomb ${ }^{2}$ [1952] into the vehicle design. The F-16-XL and X-31 research aircraft illustrate very different wing design concepts motivated by supersonic cruise research in the 1970's (F-16 XL) or maneuver aerodynamics research (X-31) in the 1990's. Finally the F-22 entered service in the early 2000's and is the current state-of-the-art air superiority maneuvering aircraft which not only incorporates high-speed super-cruise capability and high angle-of-attack maneuver capability

but also lowobservable or stealth capability into the vehicle design.

More recent aircraft design concepts have produced a class of uninhabited vehicle

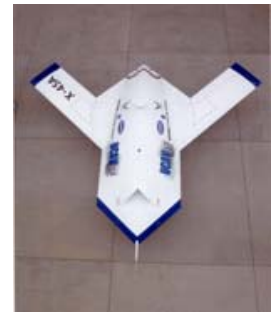

a) $X-45 A$

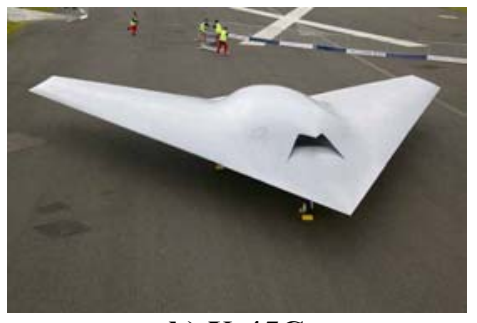

b) $\mathrm{X}-45 \mathrm{C}$

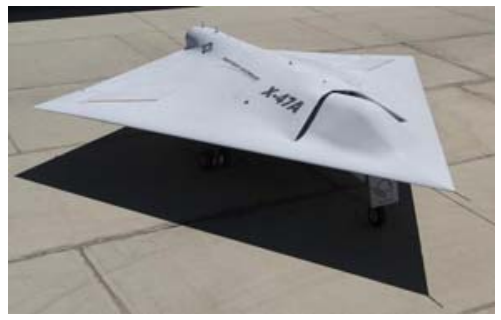

c) $X-47 A$

Figure 2. A sampling of UCAV concepts.

high subsonic or transonic flight. They include swept and moderate aspect ratio wings and are often referred to as Uninhabited Combat Air Vehicles (UCAVs). A sampling of these concepts is shown in Figure 2, and all of these vehicles incorporate stealth design principles. The X-45A represents a so-called 'lambda-wing' class of vehicle by incorporating a constant-chord outboard wing with the inner fuselage. The X-45C represents a more blended wing- 
Chicago, IL

body concept which also includes a swept and tapered wing design. Finally, the X-47A represents a slender diamond-wing design concept still with highly blended wing-body geometry.

A summary of some overall wing parameters for these aircraft is shown in Table 1. With an exception of the highly-swept F-16 XL, the wings have moderate leading-edge sweep values, from a slender wing perspective, that will be conducive to leading-edge vortex separation at maneuver angles of attack. The double values for wing leading edge sweep represent the inboard and outboard angles for the cranked F16-XL and X-31 wings. Overall the wings also fall in a moderate aspect ratio range (roughly between 2 and 3) with the exception of the higher aspect ratio X45A lambda wing and the low-aspect ratio X47A. The X-45A aspect ratio is based upon extending the leading edge sweep into the vehicle centerline with the parenthetical value based upon the entire configuration. Since slender wing principles tend to apply to the lower aspect ratio, highly-swept wings, the domain of wings exemplified by these configurations will be referred to as 'semi-slender' wings for this paper.

Table 1. Summary wing parameters.

\begin{tabular}{|l|c|c|c|}
\hline Configuration & Sweep, deg & $\begin{array}{c}\text { Aspect } \\
\text { Ratio }\end{array}$ & r/mac, \% \\
\hline F-106 & 60 & 2.1 & $0.2-0.05$ \\
\hline F-16XL & $70 / 50$ & 1.8 & $0.075-0.05$ \\
\hline X-31 & $57 / 45$ & 2.9 & Not available \\
\hline F-22 & 42 & 2.4 & Not available \\
\hline X-45A & 45 & $4.3(3.4)$ & Not available \\
\hline X-45C & 55 & 3.1 & Not available \\
\hline X-47A & 55 & 1.0 & Not available \\
\hline
\end{tabular}

This will contrast this class of wing flow from the more traditional or classical slender wing (or slender body) terminology. Another term found in the literature is 'not-so-slender' wings.

The table also shows a few leading-edge radii values normalized by the wing mean aerodynamic chord. Although not all values are available, many of these wings draw upon classical airfoil design principles such as those of the NACA 64A class of airfoils. A plot of the normalized leading-edge radius for the NACA 64A thickness profiles as a function of normalized airfoil thickness is presented in Figure 3. Data for this figure are from Abbott and von Doenhoff ${ }^{3}$ [1959], and an approximate fit to the data is also provided. In terms of practical applications for the semi-slender class of wings, the overall wing normalized thickness values tend to be $8 \%$ or less leading to small values of leading-edge radii. Observability considerations can lead to further reductions in leading-edge radii.

For these semi-slender wings, the onset and progression of separated flow aerodynamics take a number of different

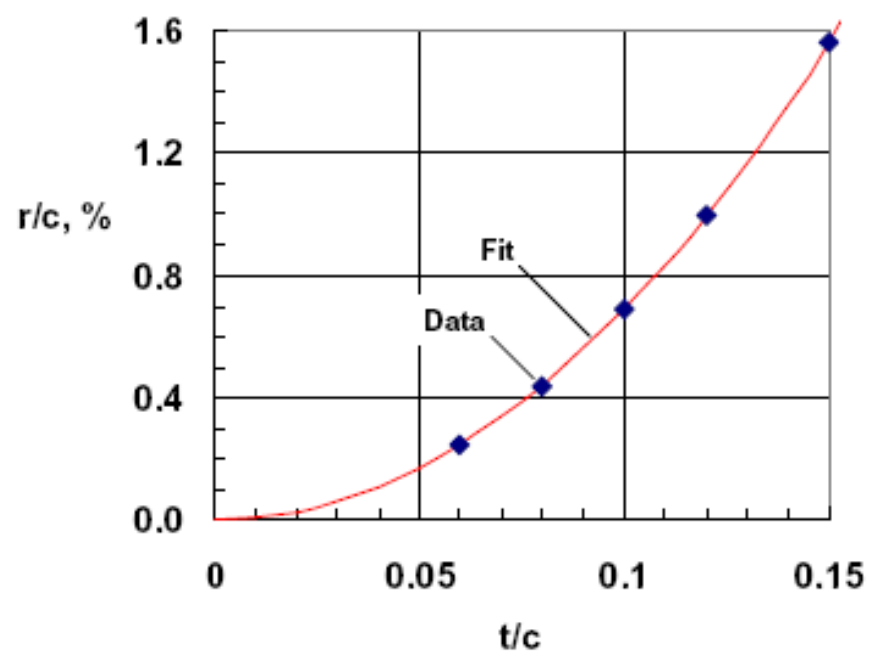

Figure 3. NACA 64A0xx leading-edge radii. characteristics due to sweep effects, leading-edge radius effects, and aspect ratio effects in association with wake interactions. The next section will review some of these effects.

\section{Select Findings from Prior Research}

Perspectives toward the onset and progression of blunt leading-edge separation for semi-slender wings can be gained by first recalling some basic features of leading-edge vortex flows from the slender sharp-edged delta wing. By virtue of the sharp leading edge, primary separation is forced to occur at the leading edge and for the slender wing this separation rolls up to form the primary leading-edge vortex. A sketch, due to Hummel ${ }^{4}$ [1978], is shown in Figure 4 that illustrates the primary leading-edge vortex. The primary vortex induces significant spanwise flow on the wing upper surface resulting in the negative suction peak also illustrated in Hummel's sketch. Past the primary suction peak the adverse spanwise pressure gradient induces separation of the spanwise boundary layer flow resulting in a secondary separation and secondary vortex. The secondary vortex induces suction outboard of the primary vortex suction peak. The primary and secondary vortices are tightly coupled, and factors affecting the secondary vortex (e.g., if the secondary separation is laminar or turbulent) also affect the primary vortex. The sharpedged slender wing continues to this day to be very useful for studying fundamentals of this flow. 
Chicago, IL

The upper surface suction due to the separation induced leading-edge vortices results in what is known as a vortex-lift increment. Polhamus ${ }^{5}$ [1966] developed a simple but very effective theoretical means to compute the vortex lift effects by his leading-edge suction analogy. This method relates the vortex lift to the attached flow leadingedge suction and was the first method to accurately predict this effect. An example is shown in Figure 5 for a sharpedge delta wing with an aspect ratio of 1.5. By Polhamus' method the lift is computed as

$$
\mathrm{C}_{\mathrm{L}}=\mathrm{K}_{\mathrm{p}} \cos ^{2} \alpha \sin \alpha+\mathrm{K}_{\mathrm{v}} \sin ^{2} \alpha \cos \alpha
$$

Here $\mathrm{K}_{\mathrm{p}}$ is the attached flow normal-force curve slope and $\mathrm{K}_{\mathrm{v}}$ is a similar term that is related to the attached flow wing leading-edge suction and governs the vortex-lift increment. Both of these terms are a function of the planform and can be determined from simple computational aerodynamics methods such as a vortex lattice. The lift equation also includes proper trigonometric relations to be applicable up to high angles of attack. The correlation shown is representative of the accuracy of this technique for estimating the lift of slender sharp-edged delta wings, and has also been achieved for fairly general

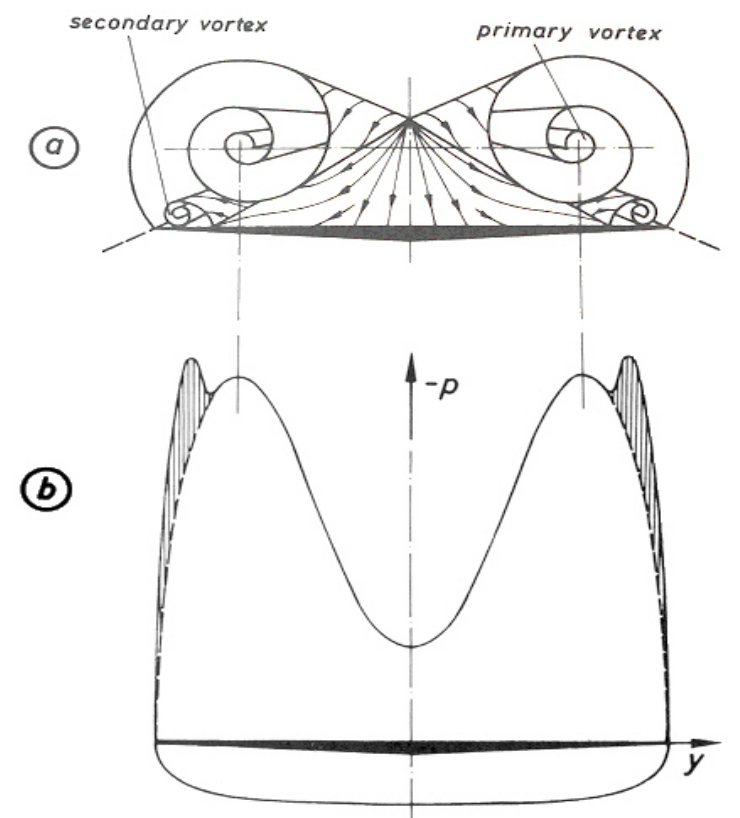

Figure 4. Sharp-edged slender wing vortex flow. Hummel $^{4}$ [1978].

planforms through extensions by $\operatorname{Lamar}^{6}$ [1974] and others. Pitching moment predictions tend to be less accurate but still useful. The large vortex lift increments like those shown are very stable and can be exploited to provide highangle-of-attack maneuver lift capability. Departures from this theory often indicate the presence of higher-order vortex effects (e.g., longitudinal curvature) or vortex state changes (e.g., vortex breakdown).

The blunt leading edge fundamentally alters this flow, and a sketch contrasting the sharp and blunt leading edge vortical flows is shown in Figure 6. Due to the leadingedge bluntness, the origin of the vortex will be displaced downstream of the apex of the delta wing. In addition, any leading-edge vortex separation will occur near, but not necessarily at the blunt leading edge. The pressure gradient between the lower-surface attachment line and the leading edge is proverse, so the separation would be expected to occur slightly beyond the leading edge on the upper surface of the wing. Details of the separation onset for the blunt leading-edge vortex could also be affected by the characteristics of the attached flow immediately ahead of the vortex. Although the sketch of the blunt leading-edge vortex separation shows the vortex origin as a point, the actual insipient separation process would be expected to be drawn out over a region of the wing.

The blunt leading edge for the swept and slender wing also leads to an onset and progression of the leading-edge vortical separation with angle of attack that is not present in the sharp-leading-edge case. For a low to moderate angleof-attack range the wing would exhibit attached flow. As

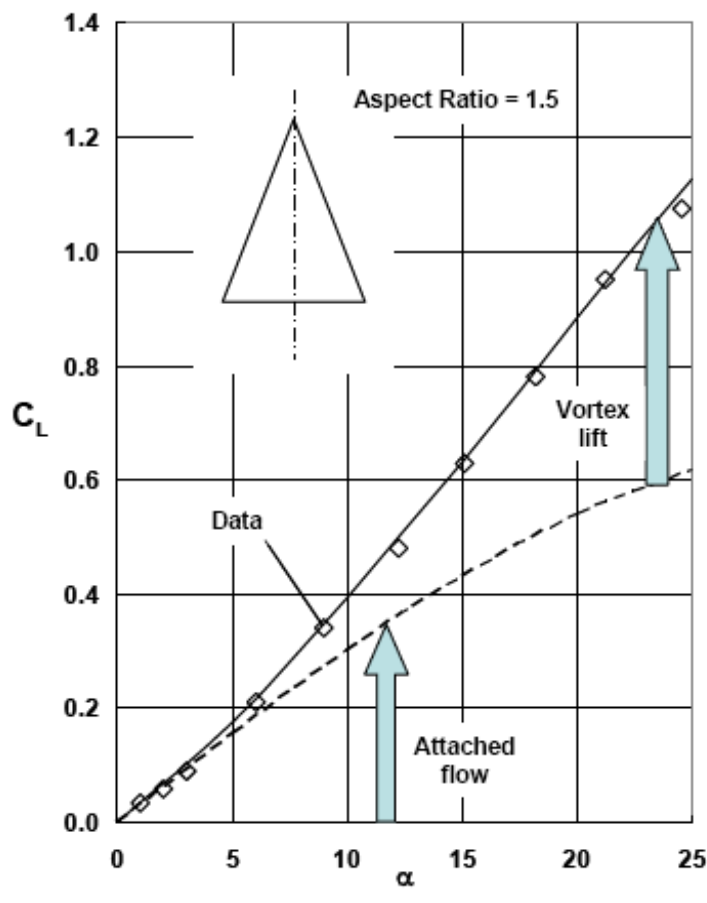

Figure 5. Application of the suction analogy. Polhamus ${ }^{5}$ [1966]. angle of attack is increased the leading-edge separation will first occur at an outboard and aft location near the trailing edge. This occurs for two reasons. First, delta wing leading-edge upwash increases from the apex to the trailing edge, and thus the local angle of attack is higher near the trailing edge. Second, the crossflow bluntness (ratio of leading-edge radius to local semispan) tends to progress from blunter to sharper values as the trailing edge 
Chicago, IL

is approached. Further increases in angle of attack cause this onset of vortical separation to progress longitudinally up the leading edge. Thus, for some angle-of-attack range the wing will exhibit partial span leading-edge vortex separation with attached flow on the upstream portion of the wing and leading edge vortex separation on the downstream portion.

An example of blunt leadingedge delta wing lift properties is shown in Figure 7. Results are for an aspect ratio 2 delta wing with a streamwise

\section{NACA-0012}

airfoil section.

The data can be found in the paper by Wick $^{8}$ [1948]. Suction analogy

estimates of the attached flow and vortex lift that would be realized by the sharp-edge case are included.

\section{$\underline{\text { Sharp leading edge }}$}

Vortex propertles vary with: - Angle of attack - Mach number - Reynolds number - etc. Primary vortex separation at leading edge
Blunt leading edge

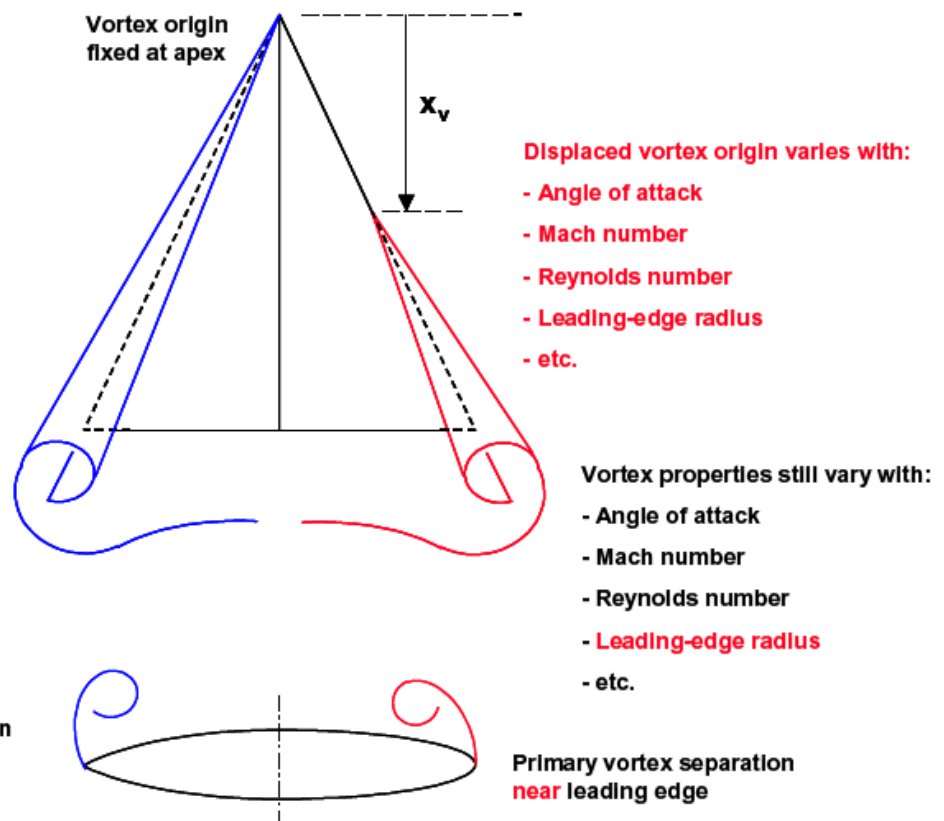

Figure 6. Comparison of sharp and blunt leading-edge separation. Luckring ${ }^{7}$ [2004].

This wing exhibits attached flow lift characteristics up to approximately 12 degrees, and additional analysis (not shown) associated with the leading-edge thrust effects indicated attached flow lift characteristics could still be present slightly above this angle. At the higher angles the data depart from the attached flow estimate in the direction of the vortex flow theory indicating the likelihood of leading-edge vortex lift effects for this wing. However, these vortex effects are much smaller than would be achieved by the sharp edge, and there are at least two reasons for this. The blunt leading edge vortex is smaller due to the part-span separation just discussed. In addition, the leading-edge bluntness weakens the vortex. The smaller and weaker vortex thus produces less vortex lift than its sharp-edged counterpart.

Because the leading edge vortex separation is now occurring from a smooth surface, the physics of this flow could be quite different from the sharp-edged case, and the strength, position, and the very existence of the vortex will be affected by leading-edge radius and will change with Mach number, Reynolds number, and angle of attack.

These effects are further complicated by the hybrid nature (attached flow inboard, partial separation outboard) of the blunt-edged slender or semi-slender wing flow. For the semi-slender wings of practical interest, it is also possible that other flow separation effects could arise on the inboard portion of the wing thereby leading to a very complex interaction of separated flows.

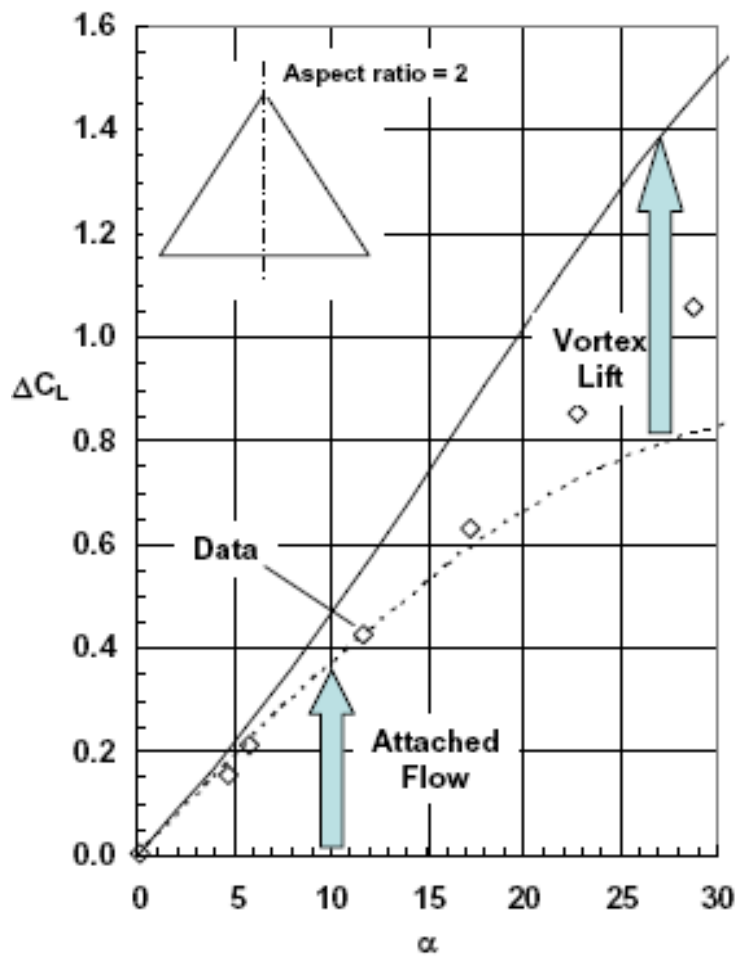

Figure 7. Blunt delta wing lift characteristics. 
Chicago, IL

Consideration of some basic wing effects pertinent to semi-slender wings will be reviewed next as regards separation onset. This will include a review of airfoil flow separation aerodynamics. The relationship between airfoil and wing aerodynamics for high aspect ratio wings is well understood. What is perhaps less appreciated is the relationship between airfoil aerodynamics and semi-slender wing aerodynamics, specifically as regards separation onset.

\section{A. Airfoil Aerodynamics}

From the interest in separated flows for this paper, it is useful to examine the types of stall developed by airfoils. These can include both leading and trailing edge separation, and a classification by Gault ${ }^{9}$ [1957] will be reviewed as reported by Polhamus ${ }^{10}$ [1996]. This analysis applies to uncambered airfoils. (See Figure 8.) In this figure, a characterization of the flow separation (not to scale) is shown above the corresponding section lift characteristics with angle of attack. Three types of stall are identified. The first type is referred to as thin airfoil stall and corresponds to an airfoil with a sharp leading edge (or a radius so small as to be effectively sharp). A laminar separation occurs followed by a turbulent reattachment forming a separation bubble. This bubble occurs essentially at any non-zero angle of
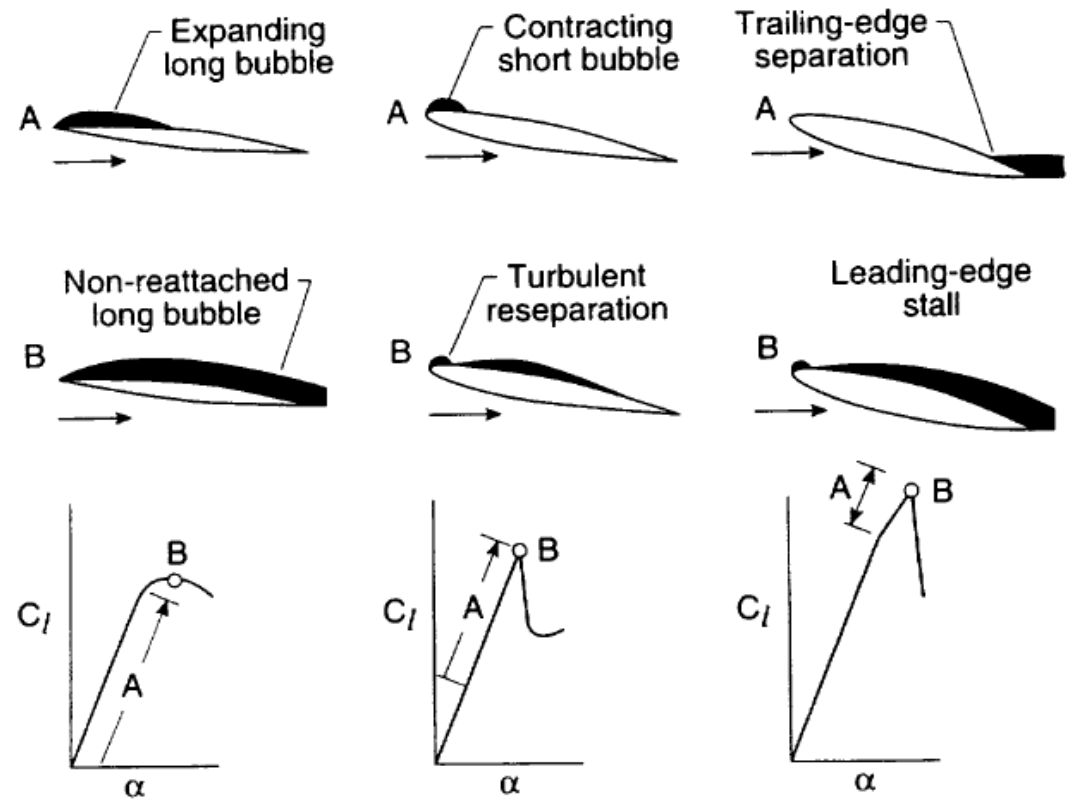

(a) Thin-airfoil stall
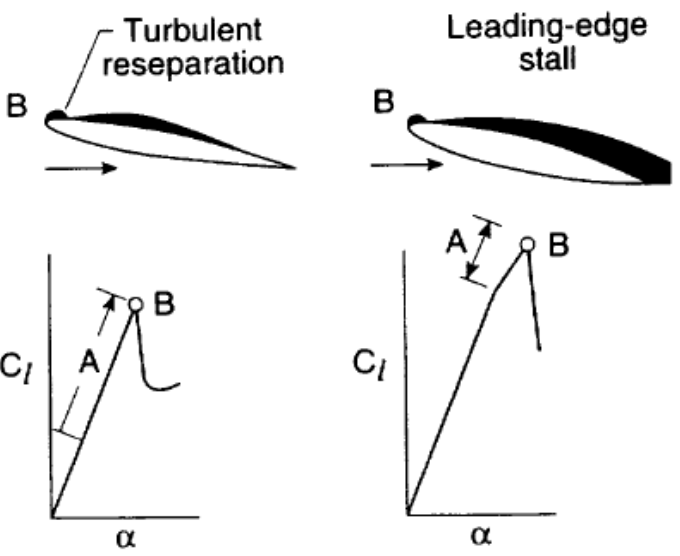

(b) Leading-edge stal attack and expands as a

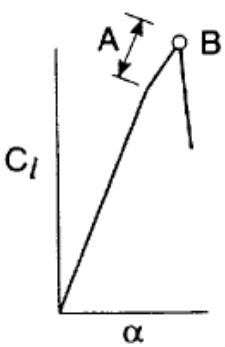

(c) Trailing edge/Leading-edge stall

long bubble on the upper surface with increasing angle of attack. As the bubble reaches the trailing edge a fairly benign stall occurs.

With an increase in leading-edge radius to relatively small values a second type of separation arises which leads to leading-edge stall. The small leading-edge radius results in very strong proverse and subsequently adverse pressure gradients to occur very close to the leading edge, and leading-edge stall is characterized by an abrupt and significant lift loss. This class of separation has a transitional short bubble very near the leading edge (less than $0.5 \%$ chord) which contracts as angle of attack increases. At critical conditions an expanding long bubble occurs resulting in the sudden loss of lift. This long bubble could be due to the short bubble bursting or due to a sudden turbulent reseparation aft of the short bubble. Either mechanism results in the sudden expansion of the turbulent long bubble and the sudden lift loss. With further increases in angle of attack the leading edge pressure gradient becomes sufficient to cause turbulent reseparation with the associated sudden lift loss.

The third stall characteristic occurs when conventional trailing-edge separation occurs in conjunction with the leading-edge separation flow physics. This class of stall is characterized by a break and an initial gradual decrease in the lift curve slope corresponding to the onset and subsequent progression of trailing edge stall. This class of stall could occur for an airfoil with a sufficiently blunt leading edge such that trailing-edge stall occurs before the leading-edge separation, in the form of the short bubble, occurs. However, leading-edge stall is still manifested, and the ensuing turbulent reseparation still results in an abrupt stall.

It should be noted that a fourth type (not shown in Figure 8) is purely trailing edge stall. Here trailing-edge separation occurs in the absence of any leading-edge separation, and the stall characteristics are gradual as the trailing edge separation point move up the airfoil. These same four classifications have been discussed by Haines ${ }^{11}$ [1994]. 
Chicago, IL

Some experimental examples of airfoil stall characteristics are shown in Figure 9. The airfoils of Figure 9a illustrate the sequence of stall characteristics, achieved for the most part due to increasing leading-edge radius. The increase in radius roughly scales with the increase in airfoil thickness. The slight break in the 64A006 data is indicative of a turbulent reseparation and long bubble which, however, does not reach the trailing edge at this relatively low angle of attack. For the $63_{1}-012$ airfoil there is a slight indication of trailing-edge stall very close to the leading-edge stall; this is difficult to see in the figure.

The airfoils of Figure 9b show the same progression in stall type but in this case the progression is primarily due to Reynolds number effects. An increase in Reynolds number will have several effects near the leading edge. Boundary layers around the leading edge will thin resulting stronger pressure gradients. The boundary layers should be better able to penetrate adverse pressure gradients. Local transition flow physics will also shift accordingly. The net effect is a trend with increasing Reynolds number that is broadly similar to the trend with increasing leading-edge radius.

Domains for the various stall types were reported by Polhamus ${ }^{10}$ [1996] in terms of leading-edge radius and chord Reynolds number. (See Figure 10.) Stall types are identified by symbols and approximate domain boundaries are shown by the hatched lines. The data come from a wide range of NACA 6series airfoils tested in the NACA Langley Low-Turbulence Pressure Tunnel (LTPT) and are for free transition. The results cover chord Reynolds numbers from 0.7 million to 30 million and normalized leading-edge radii $(\mathrm{r} / \mathrm{c})$ from 0.24 percent to 1.56 percent. The figure also illustrates cuts through the domains representing the data of Figures 9a and 9b.

Stall domains that include leading-edge separation cover a significant portion of this parameter

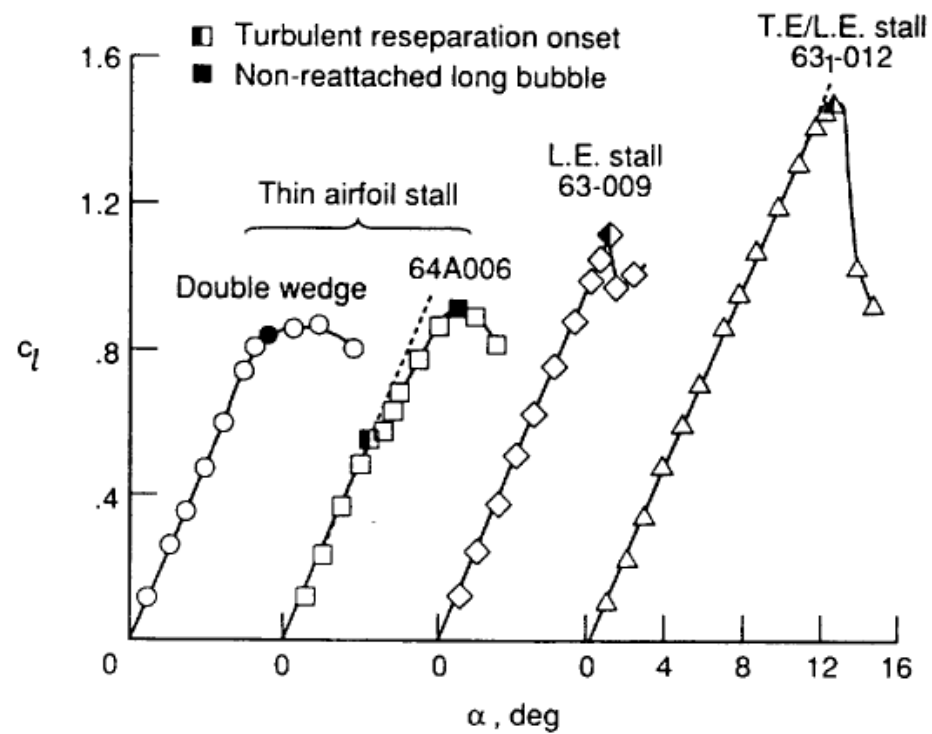

a) Leading-edge radius effects. $\mathbf{R}_{c}=\mathbf{5 . 8}$ million.

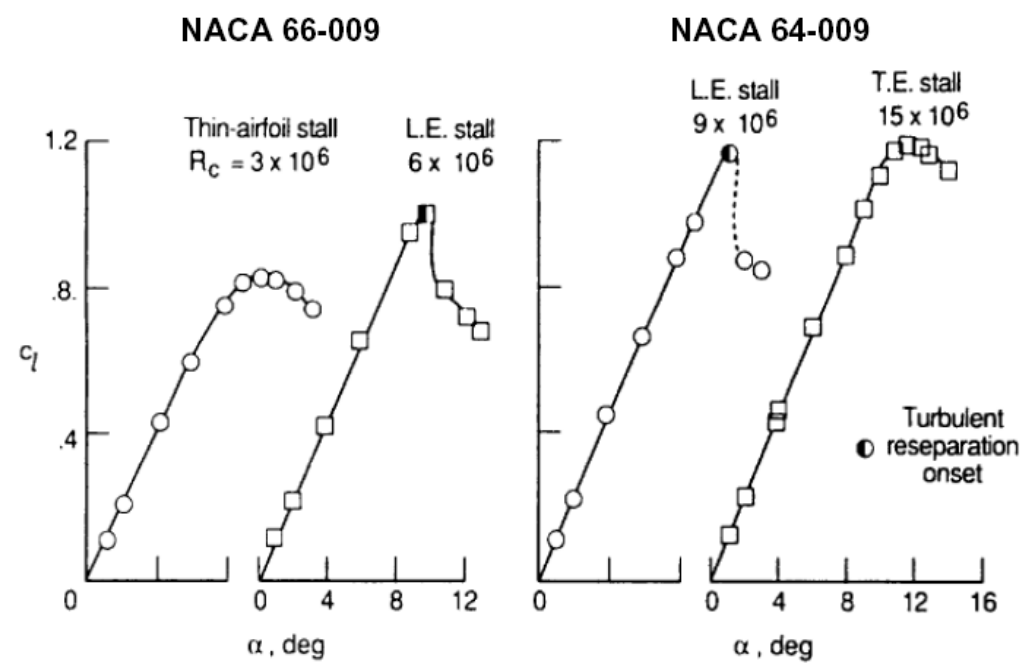

b) Reynolds number effects. $0.53 \%<r / c<0.58 \%$.

Figure 9. Leading-edge separation effects. Polhamus ${ }^{10}$ [1996]. space. In addition, the thinner airfoils (e.g., 9\% and below) of interest to this paper fall in the vicinity of the approximate boundary between leading-edge and thin airfoil stalls, thus presenting a concern from a Reynolds number scaling perspective. At the higher Reynolds numbers shown, the data imply that there may only be two domains, thin airfoil and trailing-edge stall. It must be noted that the boundaries shown for the 6-series airfoils may vary somewhat for other classes of airfoils, and they will certainly shift with camber effects as well. However, the overall suite of physics as regards separation would still be expected to manifest.

In addition to these leading-edge radius and Reynolds number effects, compressibility effects on leading-edge separation, and hence maximum section lift coefficient have been addressed by Polhamus ${ }^{10}$ [1996]. Results are shown in Figure 11. For this study NACA-0012 and ONERA D airfoils were considered. The 0012 has a leading- 
Chicago, IL

edge radius of 1.58 percent chord while the ONERA D has a leading-edge radius of 1.4 percent chord and is 10.5 prercent thick.

The NACA 0012 was tested in the LaRC LTPT which allowed for independent variation of Mach and Reynolds numbers. For the range of Reynolds numbers examined, it is anticipated that the 0012 airfoil will exhibit a combined leading-edge/trailing-edge stall. The flagged data in Figure 11a are from Loftin ${ }^{12}$ [1949] whereas the open symbols are from more recent experiments by Ladson $^{13}$ [1988]. The two data sets agree very well for the common condition $\mathrm{M}=0.15$ and show the expected increase in maximum section lift coefficient with increases in Reynolds number due to a delay in separation. The Ladson measurements expand the data set to higher Mach numbers and demonstrate a decrease in maximum lift coefficient with increasing Mach number.

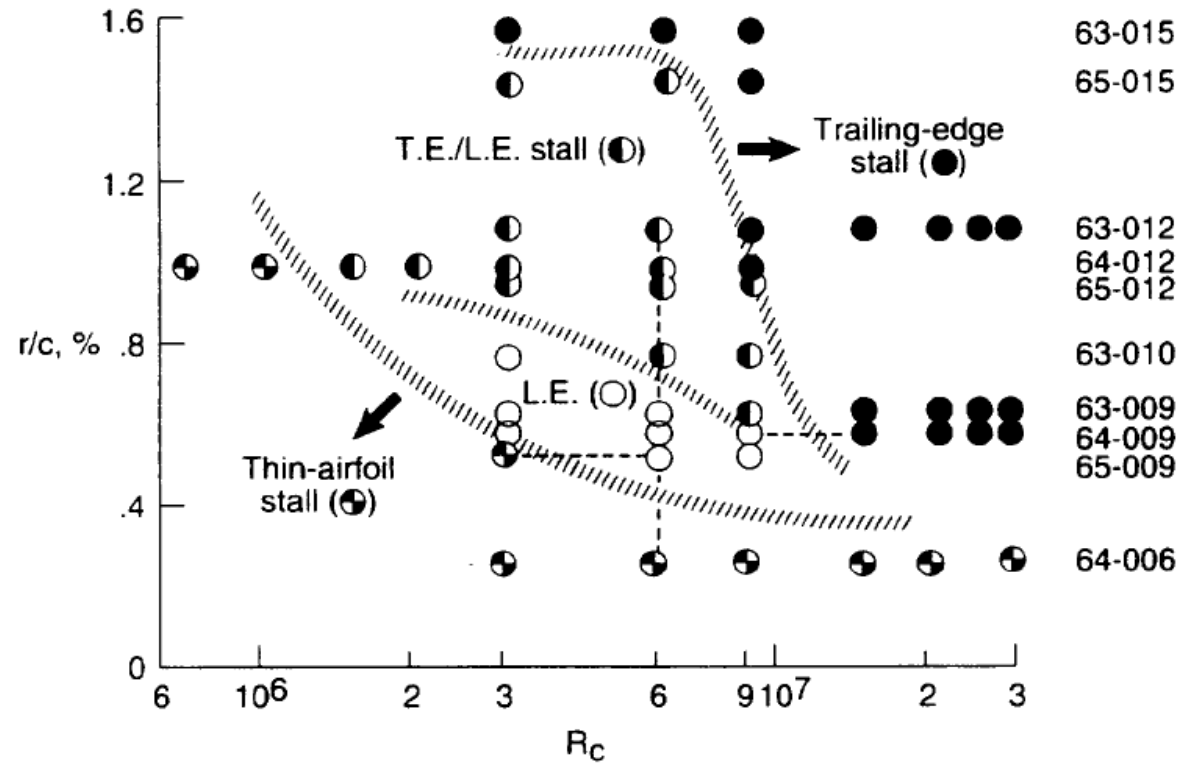

Compressibility effects

Figure 10. Stall domains. Polhamus ${ }^{10}[1996]$.

will be largest near the leading edge and will affect any detailed local flow physics (such as the contracting short bubble) and could even result in a shock-induced stall.

Dashed lines on Figure 11a show the expected maximum lift trend that would arise if Mach and Reynolds numbers were to vary simultaneously such as in an atmospheric wind tunnel (constant total pressure). This trend seems to be represented in the ONERA D data by rrlich $^{14}$ that were obtained with such a simultaneous Mach/Reynolds number variation. The LTPT results demonstrate that Mach and Reynolds numbers have opposite effects as regards the onset of separation in association with maximum section lift coefficient.

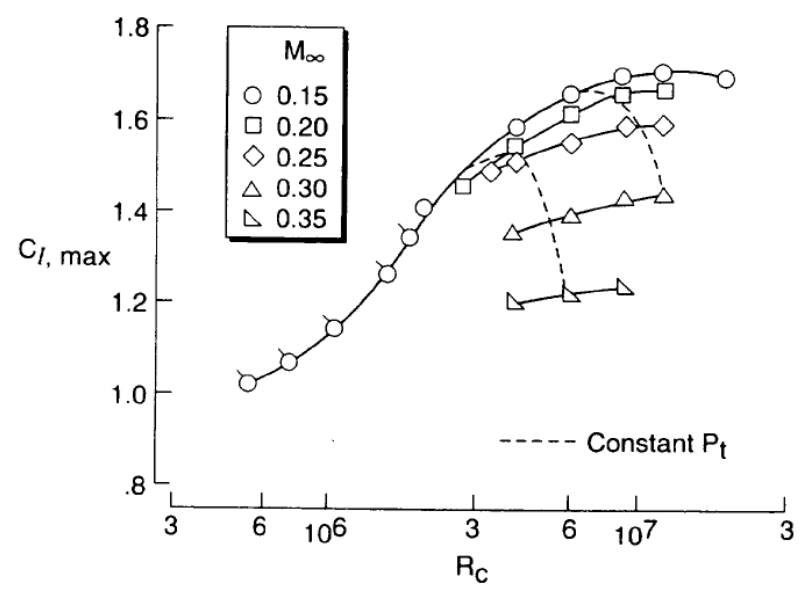

a) NACA 0012 airfoil

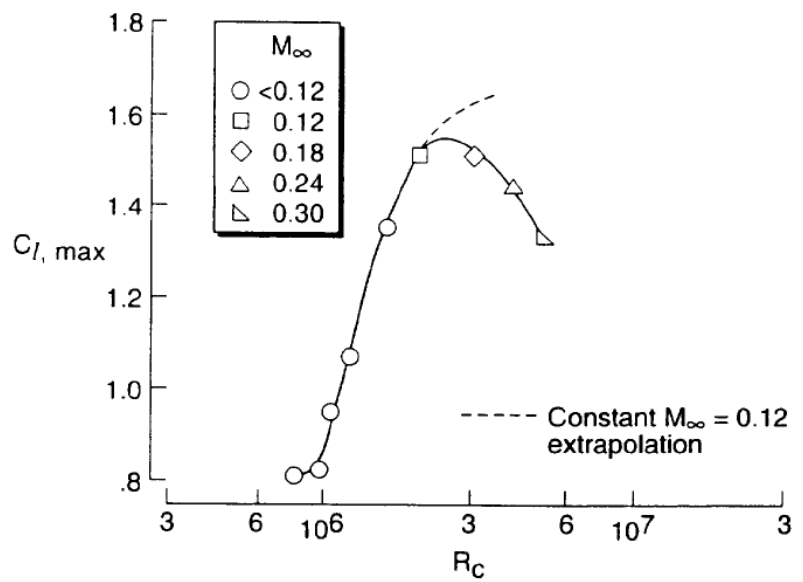

b) ONERA D airfoil

Figure 11. Mach and Reynolds number effects. Polhamus ${ }^{10}$ [1996]. 


\section{B. Finite Wing Effects}

The onset of separation can be addressed for finite wings using fundamental wing theory in conjunction with the airfoil separation characteristic from the above section. The focus for this analysis will be toward the semi-slender wings of interest to this paper. This leads to an application of airfoil separation effects to lower aspect ratio wings and higher leading-edge sweep values than perhaps is traditionally performed. Examples highlighted in this section generally fall in the airfoil domain exhibiting leading-edge separation. The preceding separated flow analysis was focused on uncambered airfoils, and the analysis of finite wing effects on separation onset and progression will be similarly restricted to uncambered wings. Additional details may be found in Polhamus ${ }^{10}$ [1996].

From the airfoil analysis the leading-edge class of stall/separation is strongly affected by the adverse pressure gradient on the upper surface very close to the leading edge. Therefore, some fundamental wing effects, such as those due to aspect ratio and sweep, on separation can be assessed in terms of the leading-edge adverse pressure gradient. Additional effects due to boundary layer state will be addressed in the subsequent section on attachment line transition.

The theoretical method chosen for the finite wing analysis is the liftingsurface theory originally developed by Multhopp $^{15}$ [1950]. A modified version of this method was developed by Lamar $^{16}$ [1968] and subsequently refined by $\operatorname{Lan}^{17}$ [1977]. As a lifting surface theory, the approach incorporates functional chordwise

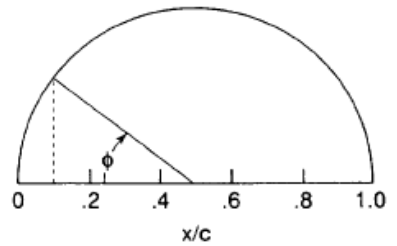

(a) $\Delta C_{p}$ formulation.

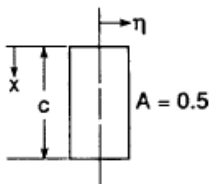

Figure 12. Lifting surface modal forms. Polhamus ${ }^{10}$ [1996]. loading distributions at discrete spanwise stations as shown in Figure 12. Modified functional forms were implemented by Lan and Lamar for numerical reasons that still retain the connection to Chebyshev polynomials. This type of theory is attractive from the standpoint of isolating angle-of-attack effects (cotangent loading) from camber effects (sin series) in the context of orthogonal functions. For the planar wings of interest to this section, it can be shown that the adverse pressure gradient very near the leading edge is dominated by the cotangent term in this theoretical approach. Whereas the wing effects on leading-edge adverse pressure gradient could be extracted from other numerical solution techniques through post processing, they are directly determined from the lifting surface theory in terms of the series coefficients $\mathrm{q}_{\mathrm{i}}$. The lifting surface theory is thus a convenience for facilitating the wing analysis.

\section{Aspect ratio}

Finite wings of course develop a wake in association with the spanwise variation of lift. The wake downwash increases downstream from the wing, leading to familiar conveniences such as Trefftz-plane induced drag formulations. Wake-induced effects propagate upstream equally well, and this leads to an induced camber effect on the finite wing in association with the spanwise variation of lift, and this effect will be larger for lower aspect ratio wings. Several key features summarized by Polhamus ${ }^{10}$ [1996] are i) induced camber effects result in a net negative load increment for the rectangular wing and ii) for a given lift

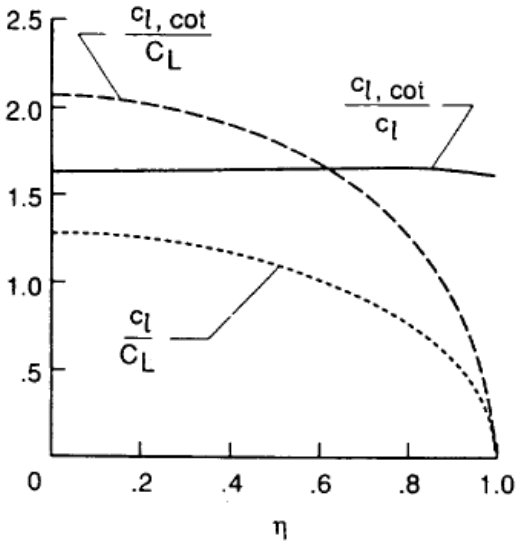

(a) Spanwise variations, $\mathrm{A}=0.5$

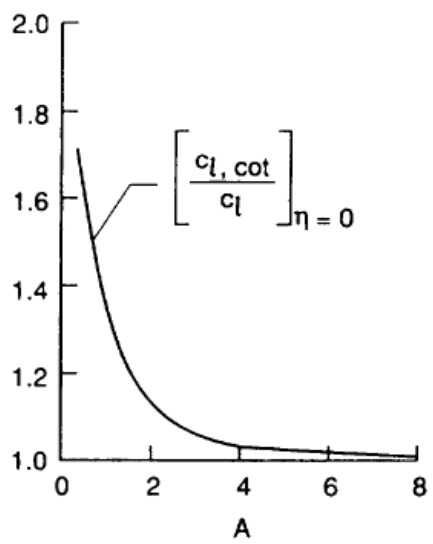

(b) Effect of $A ; \eta=0$

Figure 13. Relative loadings. Polhamus ${ }^{10}$ [1996]. 
Chicago, IL

the cotangent loading will therefore be correspondingly higher. Thus a given adverse pressure gradient will occur at a lower total lift for the finite aspect ratio wing as compared to the two dimensional case.

The basic relationship between the wing spanload and spanwise cotangent loading is shown in Figure 13. The elevated cotangent loading can be seen on the left portion, and the relative magnitude of the cotangent to total loadings at the wing centerline as a function of aspect ratio is shown on the right. Rectangular wings stall at the root section first in association with the maximum section load there. Airfoil aerodynamics will be applied to the finiteaspect-ratio wing by accounting for the three-dimensional effects predicted from the lifting-surface theory in a manner to estimate wing stall.

An application of this method to rectangular wings is shown in Figure 14 for the centerline section lift coefficient. Critical loading for stall is identified from the airfoil data, and then through the lifting-surface theory this critical loading (associated with the cotangent functional form) occurs at relatively lower overall section lift coefficients due the increasing induced camber effects with decreasing aspect ratio. The predicted three-dimensional stall condition from this method

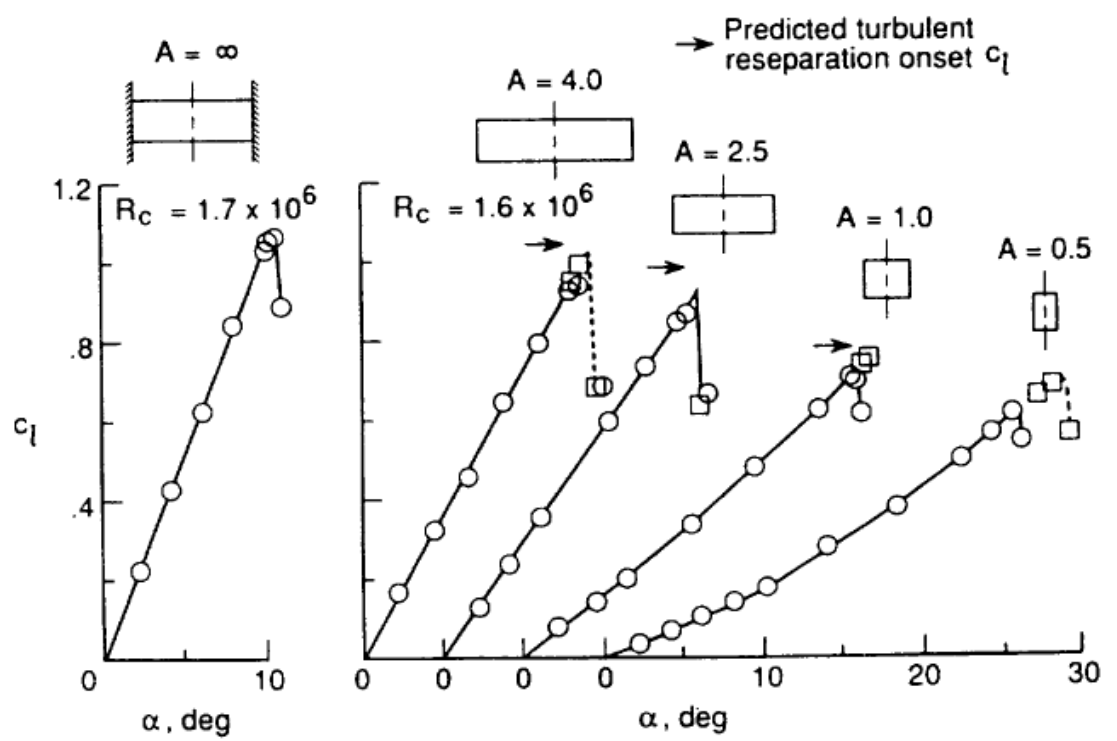

Figure 14. Predicted wing stall. RAE 101 airfoil. Polhamus ${ }^{10}$ [1996]. (arrows) agrees reasonably well with the measured three-dimensional results.

To estimate the wing maximum lift coefficient, Polhamus included vortex-lift increments expected from the side-edge vortex for rectangular wings. The side-edge vortex lift was computed for the wing at the angle of attack predicted by the above method for wing stall. Results are shown in Figure 15 for a high and a moderate aspect ratio wing. In each case the estimate of the maximum lift coefficient is surprisingly good. The airfoil Reynolds number characteristics appear to apply to these wings.

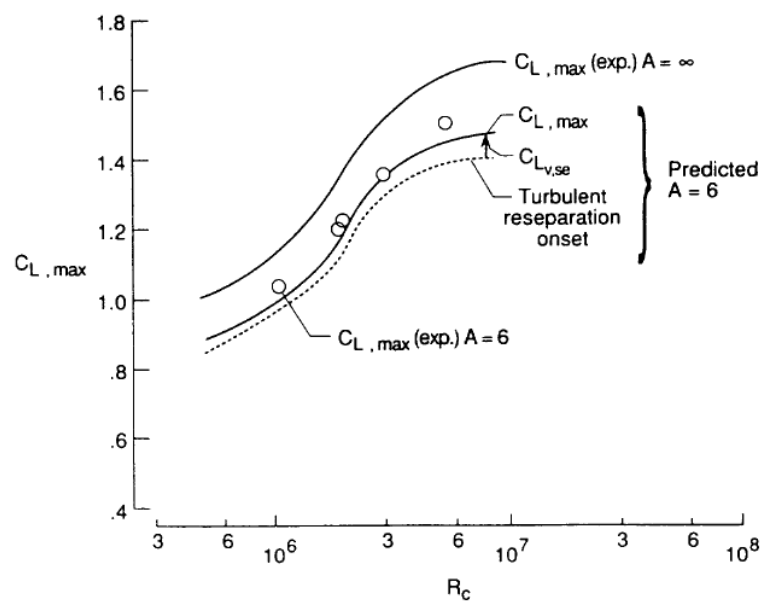

a) Aspect ratio 6 wing.

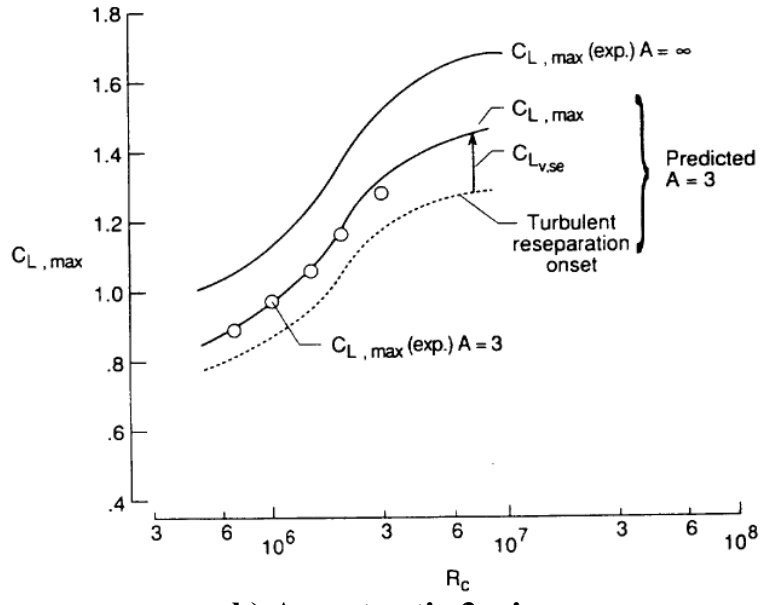

b) Aspect ratio 3 wing.

Figure 15. Predicted maximum lift coefficient. Polhamus ${ }^{10}[1996]$. 


\section{Sweep}

From basic wing theory, sweep will shift peak loading outboard, and an illustration of this effect is shown in Figure 16 along with trends in turbulent reseparation that would be expected. With sufficient sweep the separation could also result in leading-edge vortical flow. Effects of sweep on span load as well as the sectional cotangent loading are shown in Figure 17. In Figure 17a the aspect ratio effect of an elevated cotangent loading can be seen. As the wing is swept both the peak span load and peak cotangent load shift outboard, and the cotangent loading peak is seen to be both further outboard and greater in magnitude.

Results for delta wings are shown in Figure 17b. Section loadings are singular at the tip in association with the tip chord going to zero. Because the peak adverse pressure gradient near the leading edge is
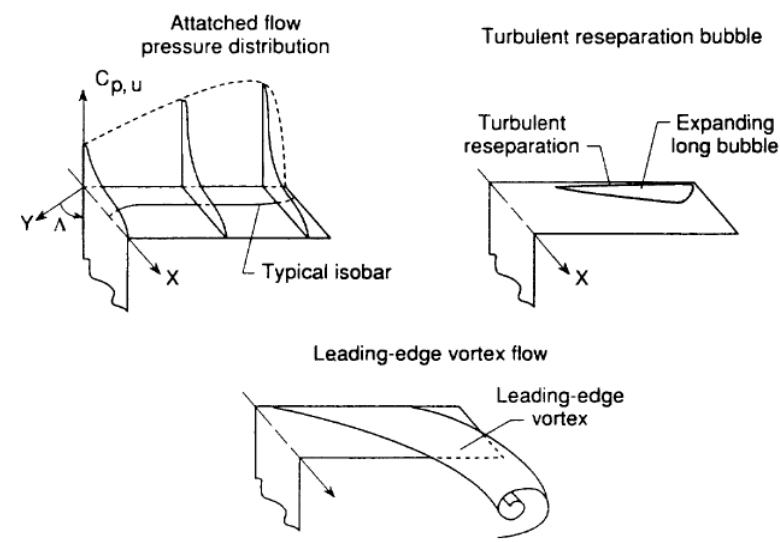

Figure 16. Swept wing flows. Polhamus ${ }^{10}[1996]$. represented through the cotangent loading, the same technique discussed above can be applied to these wings in conjunction with simple sweep theory.
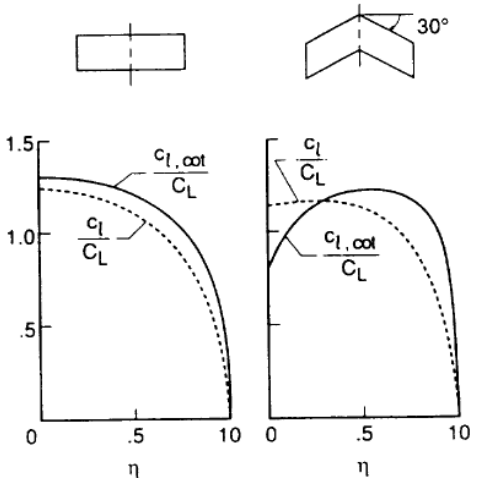

a) Sweep effect, constant chord wings.
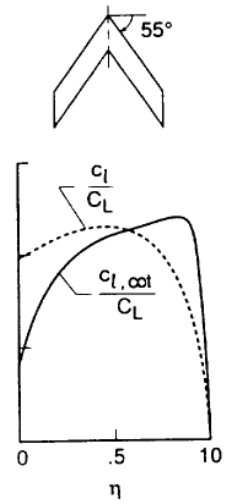

Figure 17. Effects of sweep and taper on sectional load distributions. Polhamus ${ }^{10}$ [1996].
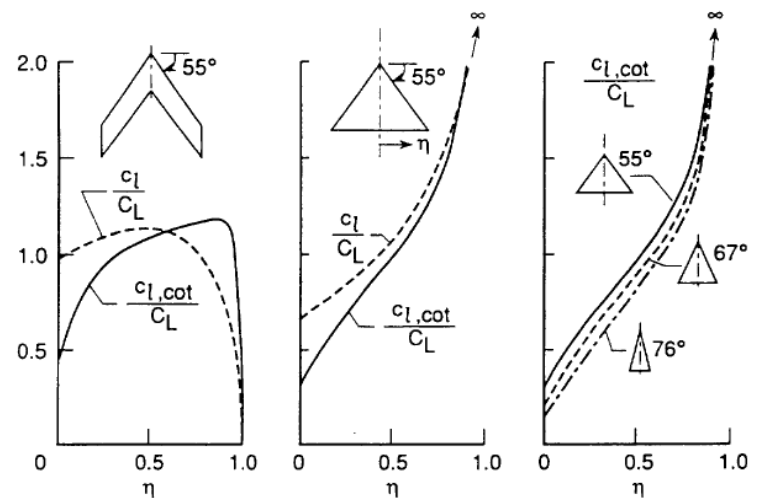

b) Delta wings.

An example is shown in Figure 18 for both a constant chord and tapered wing. The constant chord wing had an ONERA D airfoil section which was incorporated on the swept wing normal to the leading edge. Predictions by the same technique for the two sweep angles are shown by the arrows (identified with symbols that correspond to the

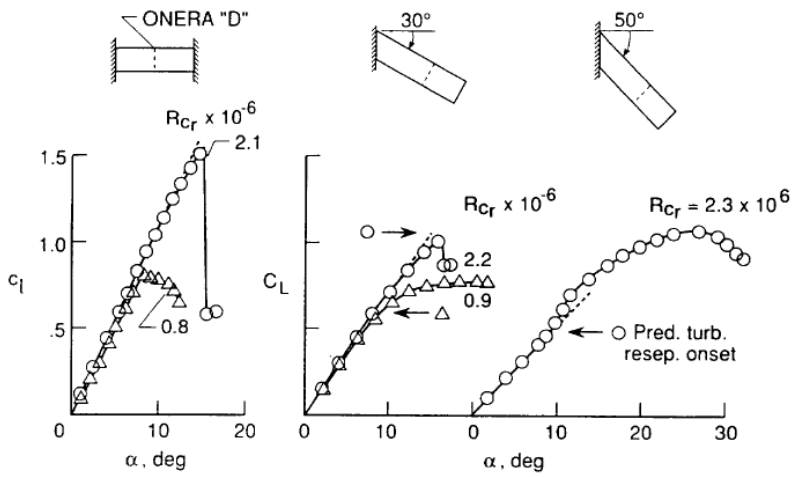

a) ONERA D airfoil, swept constant chord wing.
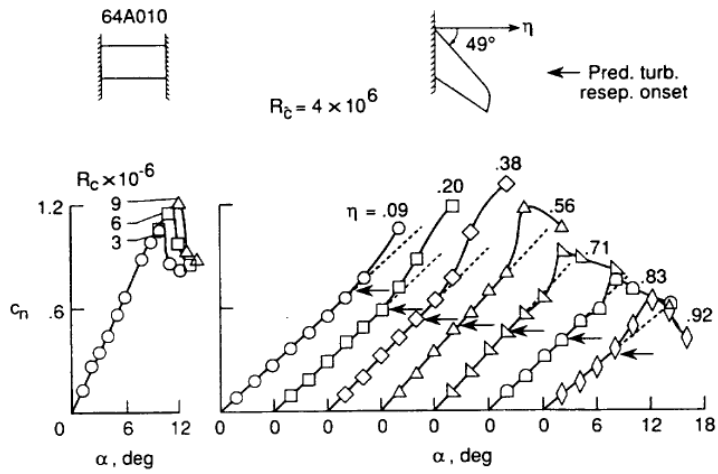

b) 64A010 airfoil, swept and tapered wing.

Figure 18. Predictions of swept-wing separation. Polhamus ${ }^{10}$ [1996].

data) and seem to correlate with lift-curve breaks in the wing data. Applications to the swept/tapered wing with a 64A010 airfoil also seem to roughly track the progression of separation up the leading edge. 
It is certainly not to be expected that a basic theoretical method as described would precisely predict onset and progression of leading-edge separation for the aspect ratios and sweeps shown in Polhamus' work. However, the degree of correlation is quite good and implies the theoretical modeling is capturing some of the dominant physics of the wing data. This application of airfoil data and simple wing theory for the purpose of predicting separation onset and progression at conditions pertinent to semi-slender wings is noteworthy.

\section{Attachment Line Transition}

Before proceeding to configuration applications some consideration to wing transition effects near the leading edge is warranted. For the onset and progression of blunt leading edge separation, with a pursuant part-span vortex separation of interest to this paper, the state of the attached-flow boundary layer leading up to separation is critical. The boundary layer around the leading edge will be fundamentally tied to the boundary layer along the lowersurface attachment line.

Poll $^{18}$ [1979] pioneered an analysis of infinite swept attachment line characteristics from a perspective of attachment line transition. Poll's sketch of the attachment line flow is shown in Figure 19. He developed what has become a universal criterion as a guideline for attachment line transition based on a local Reynolds number denoted as Rbar

$$
\begin{gathered}
\operatorname{Rbar}=\mathrm{V}_{\mathrm{a}, \mathrm{e}} \xi / v_{\mathrm{e}} \\
\xi=\left[v_{\mathrm{e}} /\left(\mathrm{dU}_{\mathrm{a}, \mathrm{e}} / \mathrm{ds}\right)_{\mathrm{a}}\right]^{1 / 2}
\end{gathered}
$$

Here $V_{a, e}$ is the velocity along the attachment line at the outer edge of the attachment line

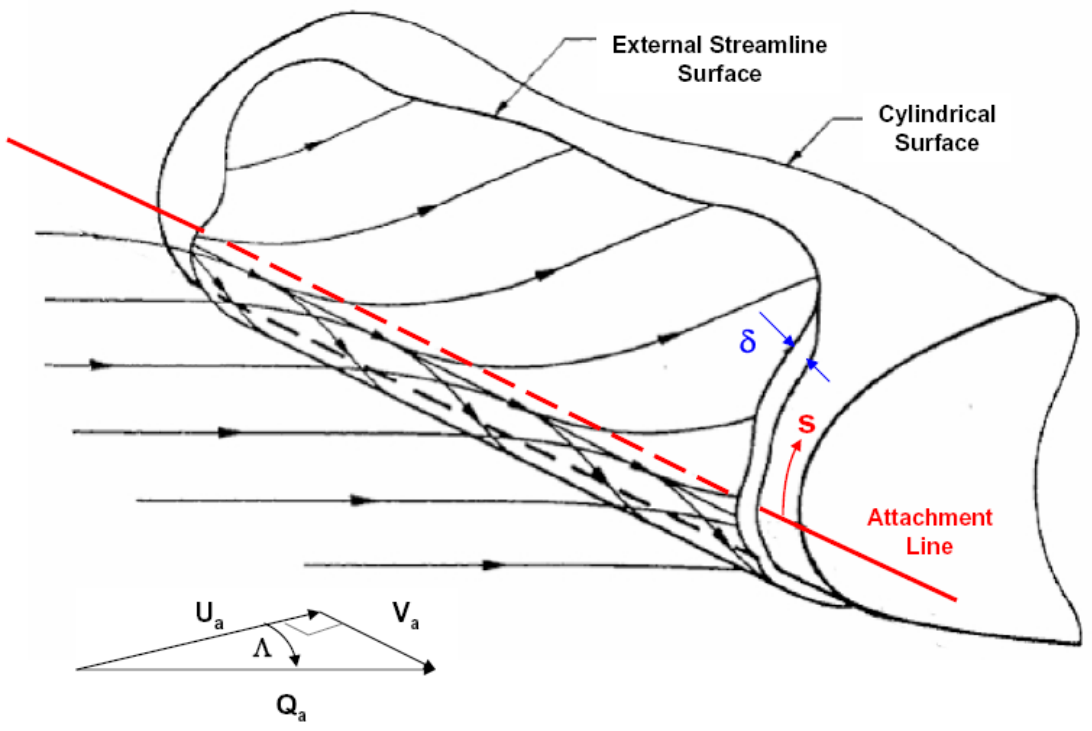

Figure 19. Attachment line flow. Poll ${ }^{18}$ [1979].

boundary layer, and the characteristics length $\xi$ is based upon parameters normal to the attachment line. In this regard $U_{a, e}$ is the velocity at the edge of the attachment line boundary layer normal to the attachment line and ' $\mathrm{s}$ ' is the surface length also in the direction normal to the attachment line. $\mathrm{U}$ and $\mathrm{V}$ are the components of the total external velocity, Q.

Criterion for the attachment line to become turbulent is given approximately by Rbar $>300$. This is a guideline that corresponds to flows with major sources of contamination. For smooth and otherwise low-disturbance surfaces Poll showed that higher values of Rbar would correspond to attachment line transition. For small disturbances, the critical value of Rbar depends on $\mathrm{d} / \boldsymbol{\xi}$ and $\boldsymbol{\ell} / \xi$, where $\mathrm{d}$ is the diameter of the small disturbance and ' $\boldsymbol{\ell}$ ' is the distance from the disturbance. For small values of $d / \xi$, values of Rbar can be as large as 700 to 800 for complete attachment line transition.

In practice, the attachment line for many swept wings can be roughly parallel to the leading edge, and thus the fundamental analysis of Poll (from a circular cylinder) can be applied to more practical wing shapes. Moreover, the necessary elements of Rbar can be inferred from static surface pressure measurements by invoking a Bernoulli assumption. Attachment line location can be determined as the location of maximum pressure. With the assumption that pressure is impressed through the boundary layer, $\mathrm{V}_{\mathrm{a}, \mathrm{e}}$ can be determined. This velocity down the attachment line can be found to not differ much from the free-stream velocity component along the attachment line. If it is assumed that $\mathrm{V}_{\mathrm{a}, \mathrm{e}}$ does not change much around the leading edge, then $\mathrm{dU}_{\mathrm{a}, \mathrm{e}} / \mathrm{ds}$ can be determined. Thus the Poll criteria can be estimated from experimental pressures, and does not require measurement of the actual velocity components.

Even when the attachment line is turbulent, the proverse pressure gradient around the leading edge can result in relaminarization. Following the two-dimensional developments of Launder ${ }^{19}$ [1969], Treadgold ${ }^{20}$ [1973] suggested the following parameter for relaminarization assessments on swept wings: 
Chicago, IL

$$
K=\left(v_{\mathrm{e}} / \mathrm{Q}_{\mathrm{e}}{ }^{2}\right) \mathrm{dQ} / \mathrm{d} \sigma
$$

Here ' $\sigma$ ' is a streamline coordinate at the edge of the boundary layer. The parameter $\mathrm{K}$ is a normalized pressure gradient which, when sufficiently large, can lead to relaminarization. Guidelines indicate that $\mathrm{K}$ needs to exceed $5 \mathrm{x}$ $10^{-6}$ for relaminarization, and that departures from fully turbulent flow (partial relaminarization) can occur for $\mathrm{K}$ exceeding $2 \times 10^{-6}$. A Bernoulli relationship is used to relate the total velocity at the edge of the boundary layer to the static surface pressure, and thus $\mathrm{K}$ can also be determined from pressure measurement so long as the measurement density is sufficient to estimate the streamline velocity gradient. Two examples of applying these criteria to leadingedge attachment line flows follow.

The first example is for a cropped delta wing reported by Ashill $^{21}$ [1990]. The configuration was a semispan model with a 60 degree swept cropped delta wing. The wing was cambered and $4 \%$ thick with a streamwise leadingedge radius of 0.13 percent centerline chord that was nearly constant in the spanwise direction. Data were obtained at subsonic and supersonic conditions and for a range of Reynolds numbers and
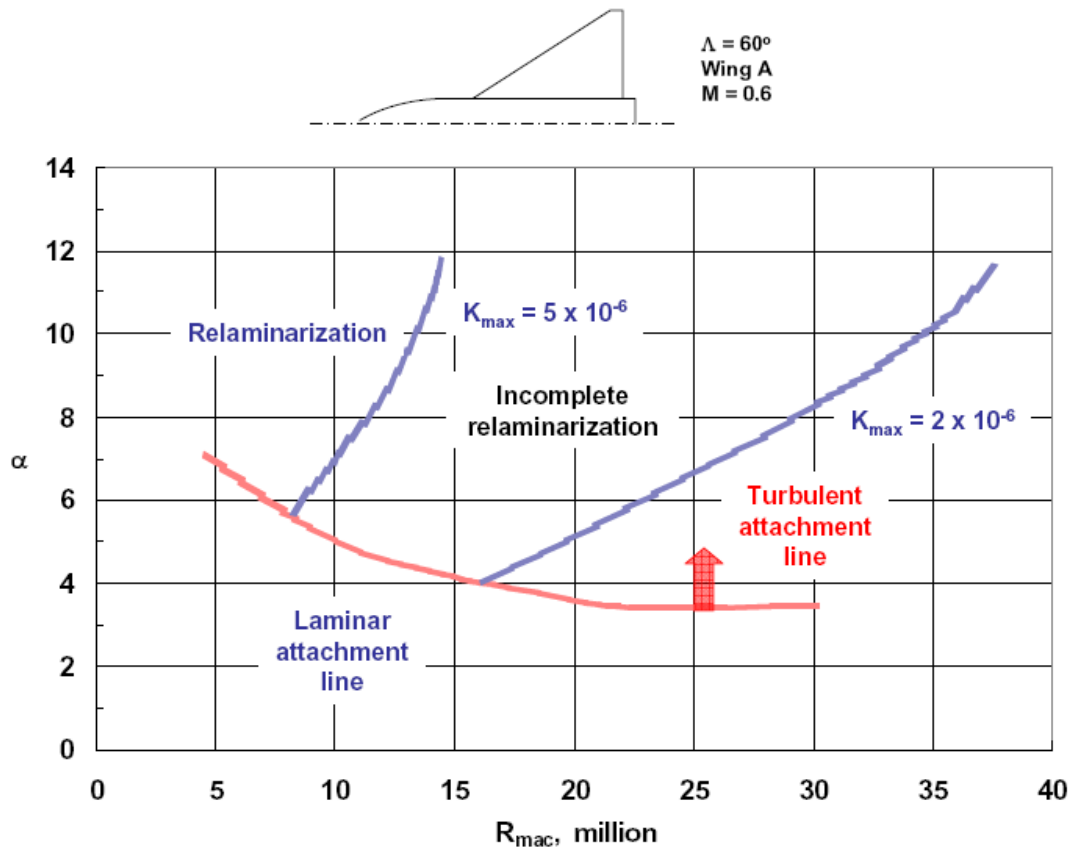

Figure 20. Attachment line contamination and relaminarization boundaries. Ashill ${ }^{21}$ [1990]. angles of attack.

An example of Ashill's analysis is presented in Figure 20. Here domain boundaries are illustrated in an angle-of-attack and Reynolds number parameter space for a position about $70 \%$ down the leading edge. The red curve shows the approximate boundary between laminar and turbulent attachment lines for the position chosen. (The authors do not report the specific criterion for this curve, but it is presumed to be a critical Rbar value following Poll). In addition, approximate boundares for the two values of $\mathrm{K}_{\max }$ (in the vicinity of the leading edge) indicate domains for which subsequent relaminarization might be expected. The boundaries

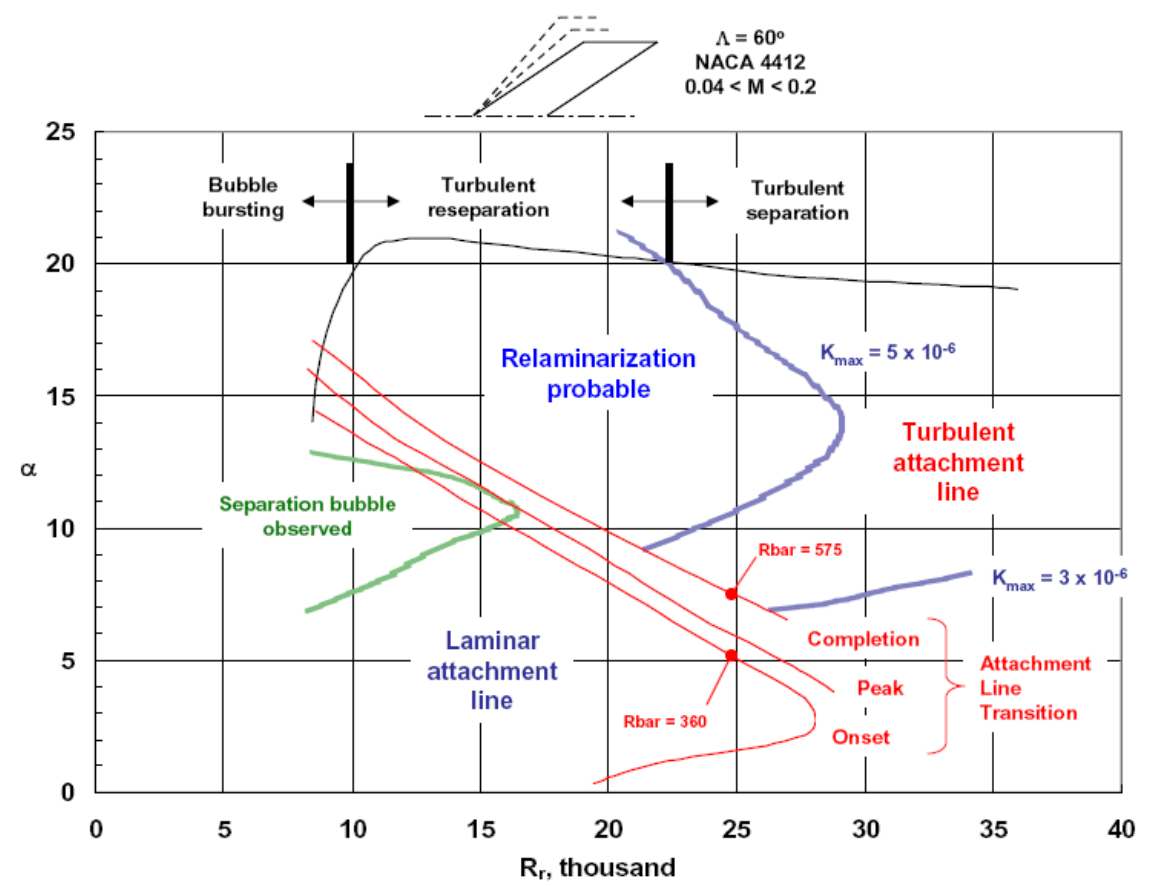

Figure 21. Flow physics boundaries for leading-edge flow characteristics. Ashill $^{22}$ [1993]. 
were inferred from static surface pressures as described above. This experimental analysis provides considerable insight to leading-edge flow characteristics that can be critical to leading-edge separation.

A second example from Ashill ${ }^{22}$ [1993] is for a constant chord swept wing tested in the 13x9-foot low-speed atmospheric tunnel at Bedford. The wing had a NACA 4412 airfoil normal to the leading edge with a leading-edge radius of $1.58 \%$ chord. The wing was tested with sweep angles of $45^{\circ}, 53^{\circ}$, and $60^{\circ}$, and Ashill's leading-edge analysis was reported for the $60^{\circ}$-swept case. Reynolds number variations were achieved through free stream velocity variation, so some compressibility effects will be included in the measurements.

An example of Ashill's analysis for this case is shown in Figure 21, and the results are reported to be representative of the mid-span portion of wing. The attachment-line transition boundaries are based on hot-film measurements. Values of Rbar are also shown for a few conditions, and overall agree with the guidelines established by Poll. Above the red line, the attachment line would be turbulent. The $\mathrm{K}_{\max }$ guidelines are also indicated to show conditions, after attachment line transition, where relaminarization could occur. A boundary for which a laminar short-bubble separation was apparent in the experimental pressures is also shown. Finally, at the top of the figure the locus for the onset of the collapse of the leading-edge suction peak is shown in black. Three mechanisms are shown for this event spanning bubble bursting, turbulent reseparation, and turbulent separation.

This analysis provides a more detailed assessment of the leading-edge separation flow physics than shown in the previous example. This also provides considerably more insight into the flow details than was inferred from the aggregate wing analysis developed by Polhamus.

\section{Basic Applications}

Two basic application studies are summarized that draw upon some of the results discussed above. The first was conducted with a generic wing-body geometry while the second used a delta wing. Both investigations are experimental and address Reynolds number and leading-edge radius effects.

1. Generic Wing-Body

A generic wing-body configuration was tested in the LaRC LTPT as reported by Henderson ${ }^{23}$ [1976]. The model had leading-edge sweep angles of 61.7, 64.6, and 67.0 degrees and trailing-edge sweep angles of 0 and 40.6 degrees. The wing could have sharp, small or large leading-edge radii that resulted in a range of edge radii (normal to leading edge) of 0.1 to 0.6 percent mean aerodynamic chord among the configurations. A sketch of these models is shown in Figure 22. Data were obtained at Mach numbers below 0.3 and for Reynolds numbers, based on mean aerodynamic chord, between 1.5 million and 20.4 million.

Henderson presented an analysis for the onset and

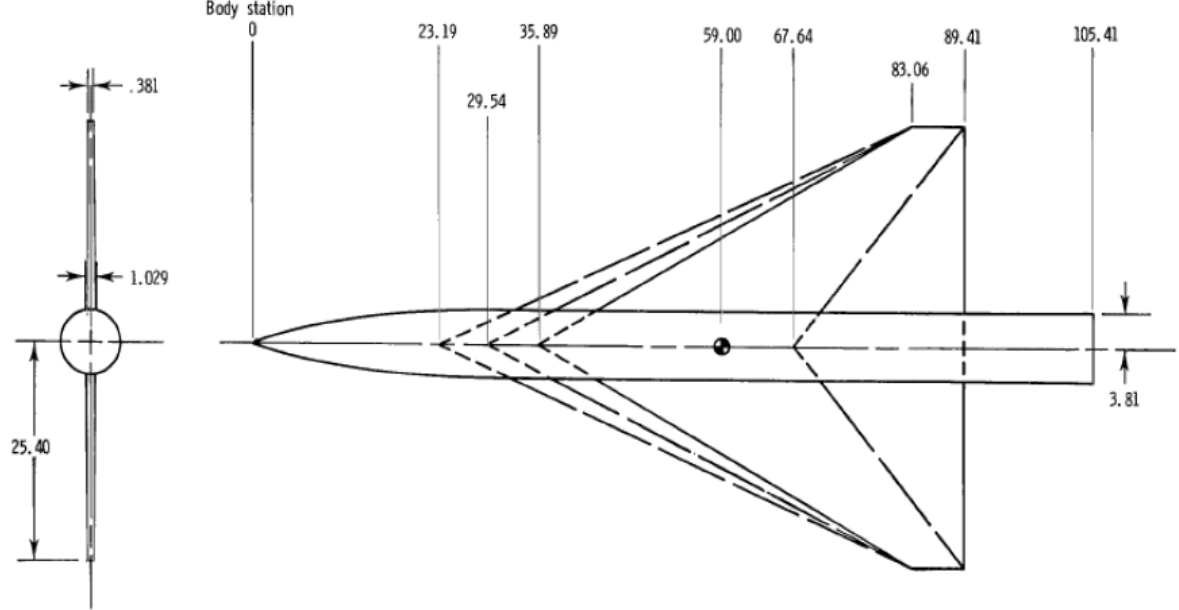

Figure 22. Basic wing-body configuration. Henderson ${ }^{23}$ [1976]. progression of blunt leading-edge separation based upon normal and suction force coefficients. The normal force analysis drew upon the suction analogy such that a vortex-related normal force increment could be estimated by

$$
\begin{gathered}
\mathrm{C}_{\mathrm{N}, \mathrm{p}}=\mathrm{K}_{\mathrm{p}} \cos \alpha \sin \alpha \\
\mathrm{C}_{\mathrm{N}, \mathrm{v}}=\mathrm{C}_{\mathrm{N}, \exp }-\mathrm{C}_{\mathrm{N}, \mathrm{p}}
\end{gathered}
$$

This is similar to the delta wing analysis presented earlier in this paper. The suction force was estimated in the usual way:

$$
\mathrm{C}_{\mathrm{S}}=\left[\mathrm{C}_{\mathrm{L}} \tan \alpha-\left(\mathrm{C}_{\mathrm{D}}-\mathrm{C}_{\mathrm{D}, \mathrm{o}}\right)\right] \sec \Lambda \cos \alpha
$$


Chicago, IL

At low angles of attack, with attached flow, the vortex normal force will be essentially zero and the suction force will be the 'full-suction' value for this configuration. At higher angles of attack, with part-span vortex separation, the vortex normal force will provide the vortex increment realized over the aft portion of the wing while the suction force will provide the residual suction force realized mostly from the attached flow over the forward portion of the wing.

An example of this analysis is shown in Figure $\mathbf{2 3}$ for the three radii tested at a relatively high Reynolds number

$$
\begin{aligned}
& R_{\text {mac }}=10 \text { million } \\
& \Lambda_{\mathrm{le}}=61.7^{\circ} \\
& \Lambda_{\mathrm{te}}=0
\end{aligned}
$$
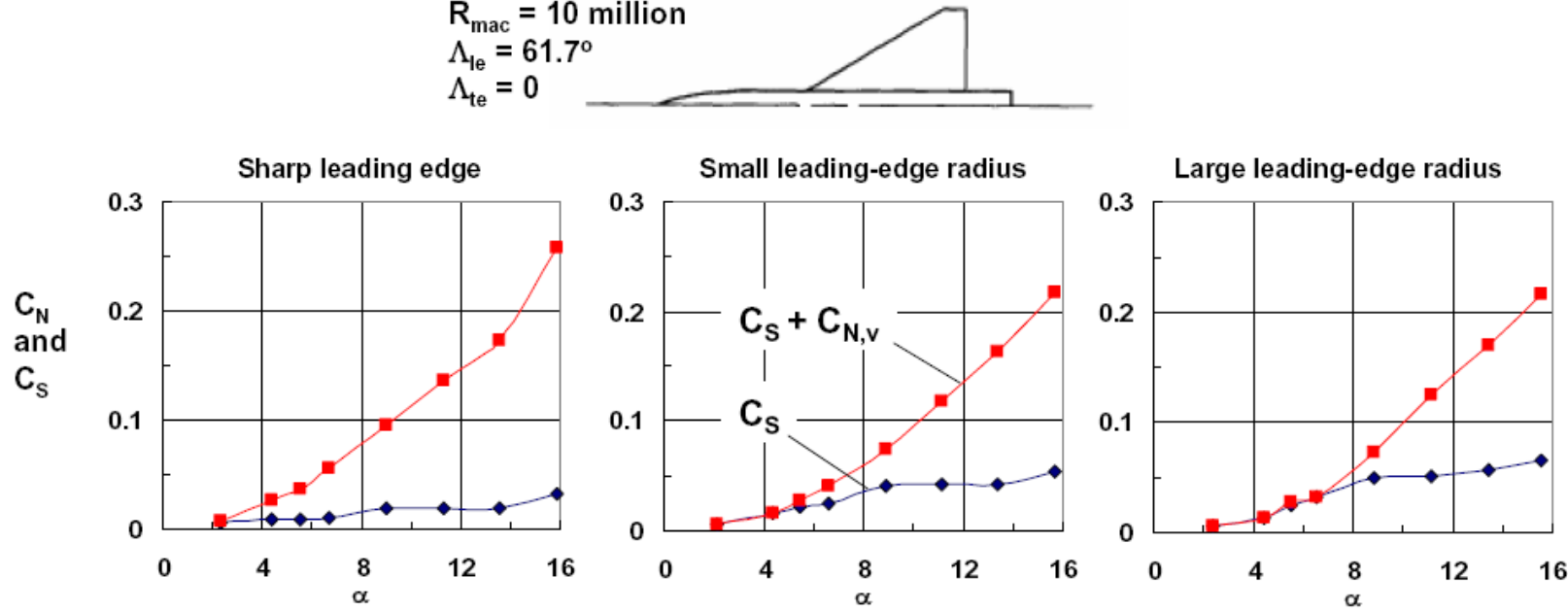

Figure 23. Leading-edge radius effect. Henderson ${ }^{23}[1976]$.

condition. By Henderson's analysis the suction and vortex normal force coefficients are combined at each angle of attack. The break between these two components represents the onset of vortex normal forces and simultaneous loss of attached flow leading edge suction. The figure shows the delay in separation onset in association with increasing leading-edge radius. This particular force accounting is consistent with the theoretical basis of the suction analogy.

A second example is shown in Figure $\mathbf{2 4}$ for one configuration (with the blunter leading edge) at two Reynolds numbers. Here the analysis shows the delay of separation with an increase in Reynolds number. Suction analogy estimates on these figures are fairly close to the experimental sum of residual suction and vortex normal force.

$$
\begin{aligned}
& \text { Large leading-edge radius } \\
& \Lambda_{\mathrm{le}}=61.7^{\circ} \\
& \Lambda_{\mathrm{te}}=0
\end{aligned}
$$
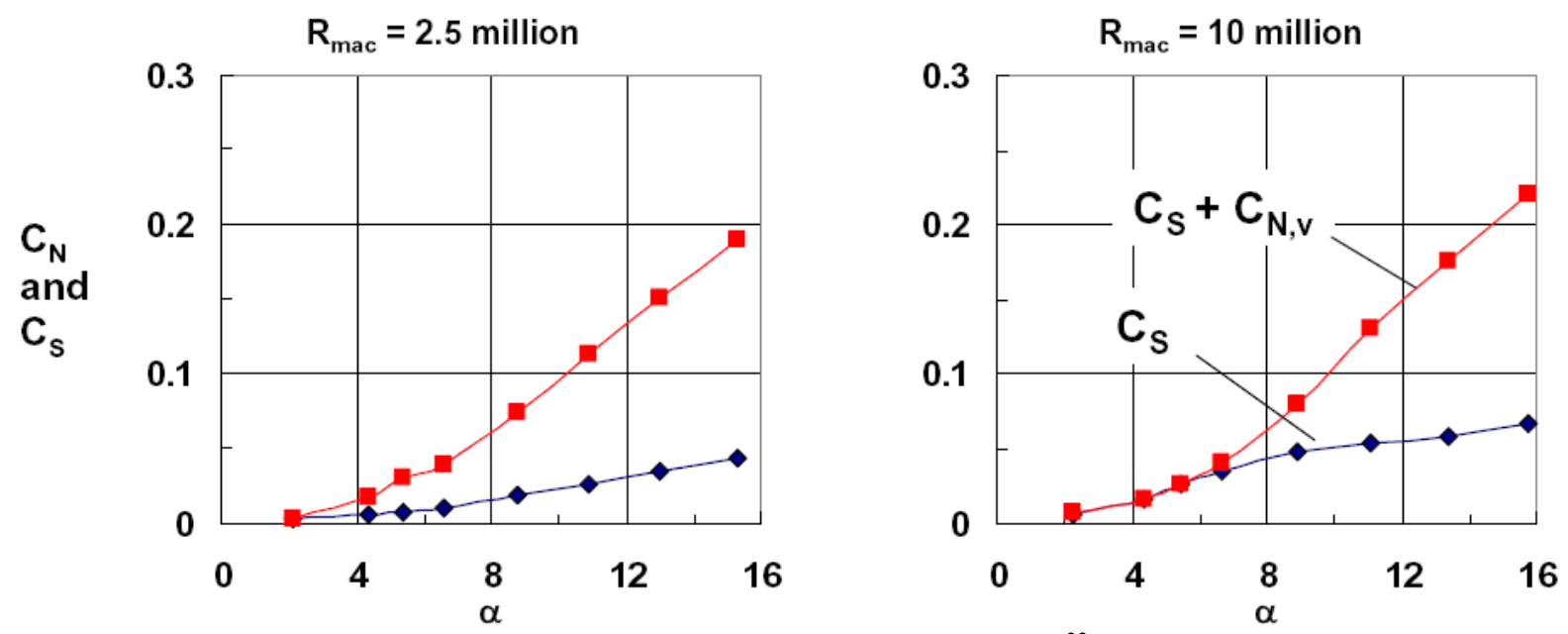

Figure 24. Reynolds number effect. Henderson ${ }^{23}[1976]$. 


\section{Delta wing}

A delta wing experiment was conceived for testing in the National Transonic Facility (NTF) as reported by Luckring $\left[2002^{24}, 2003^{25}, 2004^{26}, 2004^{7}\right]$. The wing had a $65^{\circ}$ leading edge sweep and four streamwise leading-edge radii of $0,0.05,0.15$, and 0.30 percent mean aerodynamic chord. Although this delta wing would be considered more a slender wing than a semi-slender wing, the study explicitly addresses the onset and progression of blunt leading-edge separation including independent Mach and Reynolds number effects. The tests spanned Mach numbers from 0.4 to 0.9 and Reynolds numbers, based on mean aerodynamic chord of 6 million to 120 million. A photograph of the model in NTF is shown in Figure 25. Data are documented in reports by $\mathrm{Chu}^{27}$ [1996].

Sample results for the effect of bluntness are shown in Figure 26. Results correspond to a Mach number of 0.4, a Reynolds number of 6 million, and an angle of attack of 13 degrees. The data on the left show fairly conventional

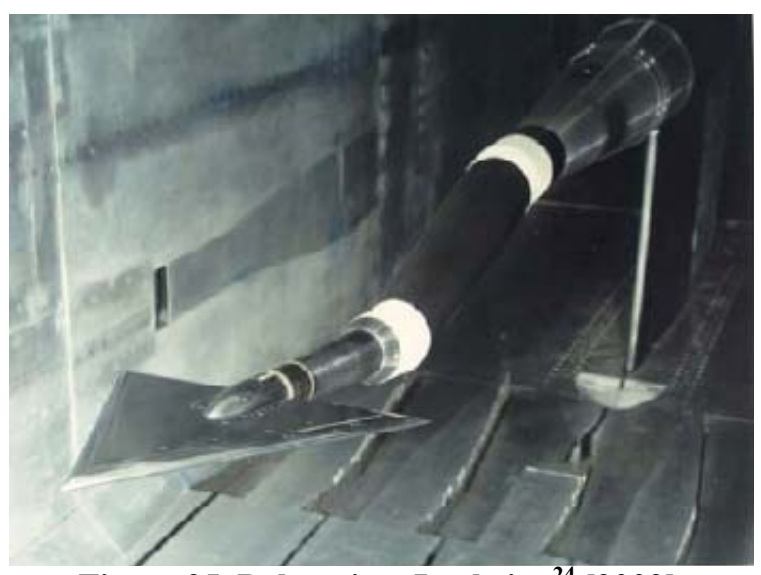

Figure 25. Delta wing. Luckring ${ }^{24}$ [2002]. leading-edge vortex flow for the sharp-edge case. The data on the right illustrate the part-span vortex separation which has already initiated at the 0.4 chord station. Pressures in the inset show a double bump in the blunt edge case, and this inner suction was determined to be a second, co-rotating vortex, inboard of the more conventional outer primary vortex. This flow structure became a focus of an RTO Task group to be discussed later in this report.

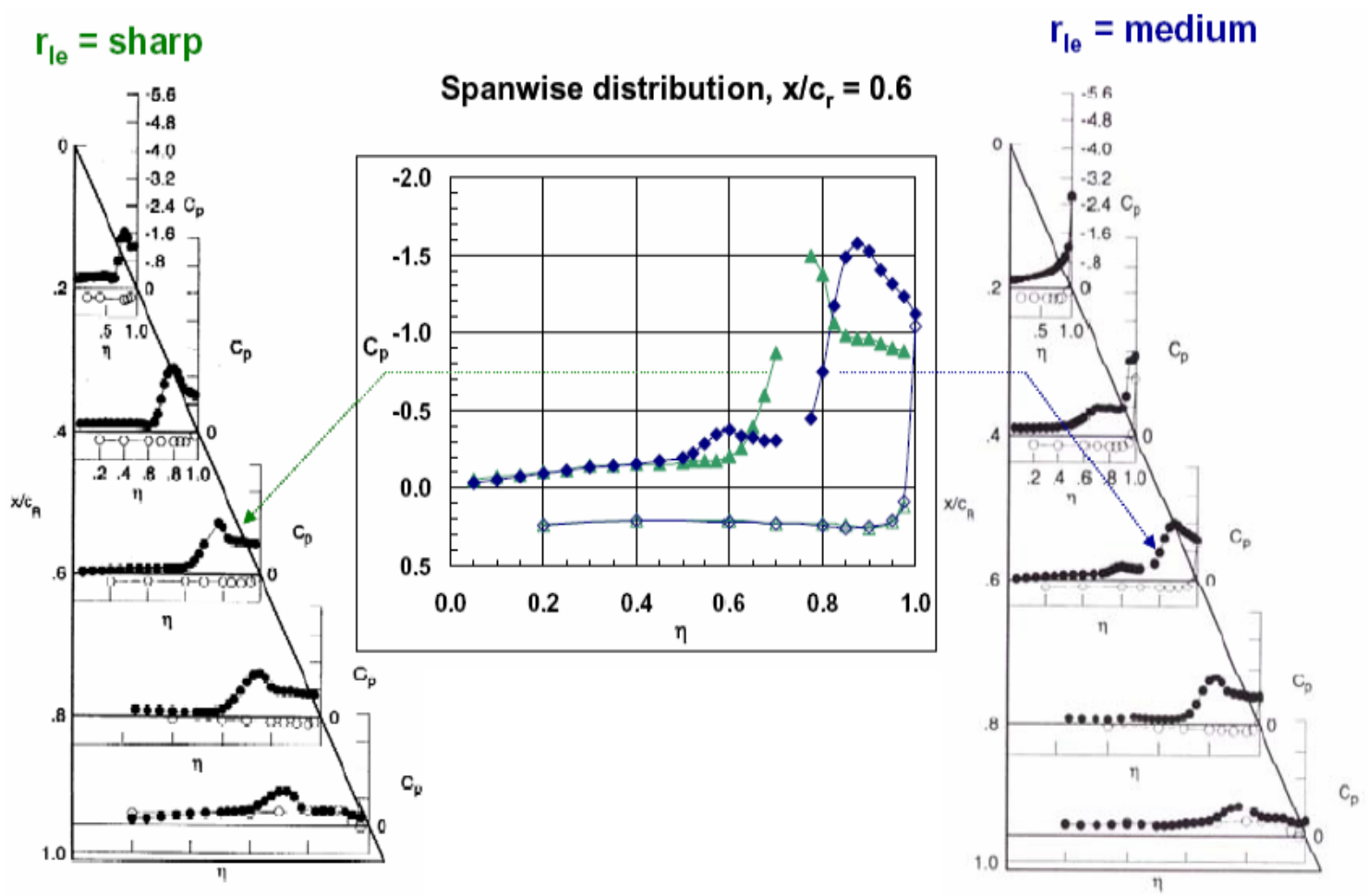

Figure 26. Leading-edge bluntness effect. $M=0.4, R_{\operatorname{mac}}=6$ million, $\alpha=13^{\circ}$. Luckring ${ }^{24}[2002]$.

An example of Reynolds number effects is presented in Figure $\mathbf{2 7}$ for the wing with the medium leading-edge radius at a Mach number of 0.4 and an angle of attack of 13 degrees. The pressure distributions clearly show the delay of blunt leading-edge separation with the increase of Reynolds number. For the conditions shown, the leadingedge separation has shifted aft by approximately 20 percent chord. An example of Mach number effects is presented in Figure 28 for the wing with the medium leading-edge radius at a Reynolds number of 60 million and an angle of 
attack of 13 degrees. The pressure distributions clearly show the promotion of blunt leading-edge separation with the increase in Mach number.
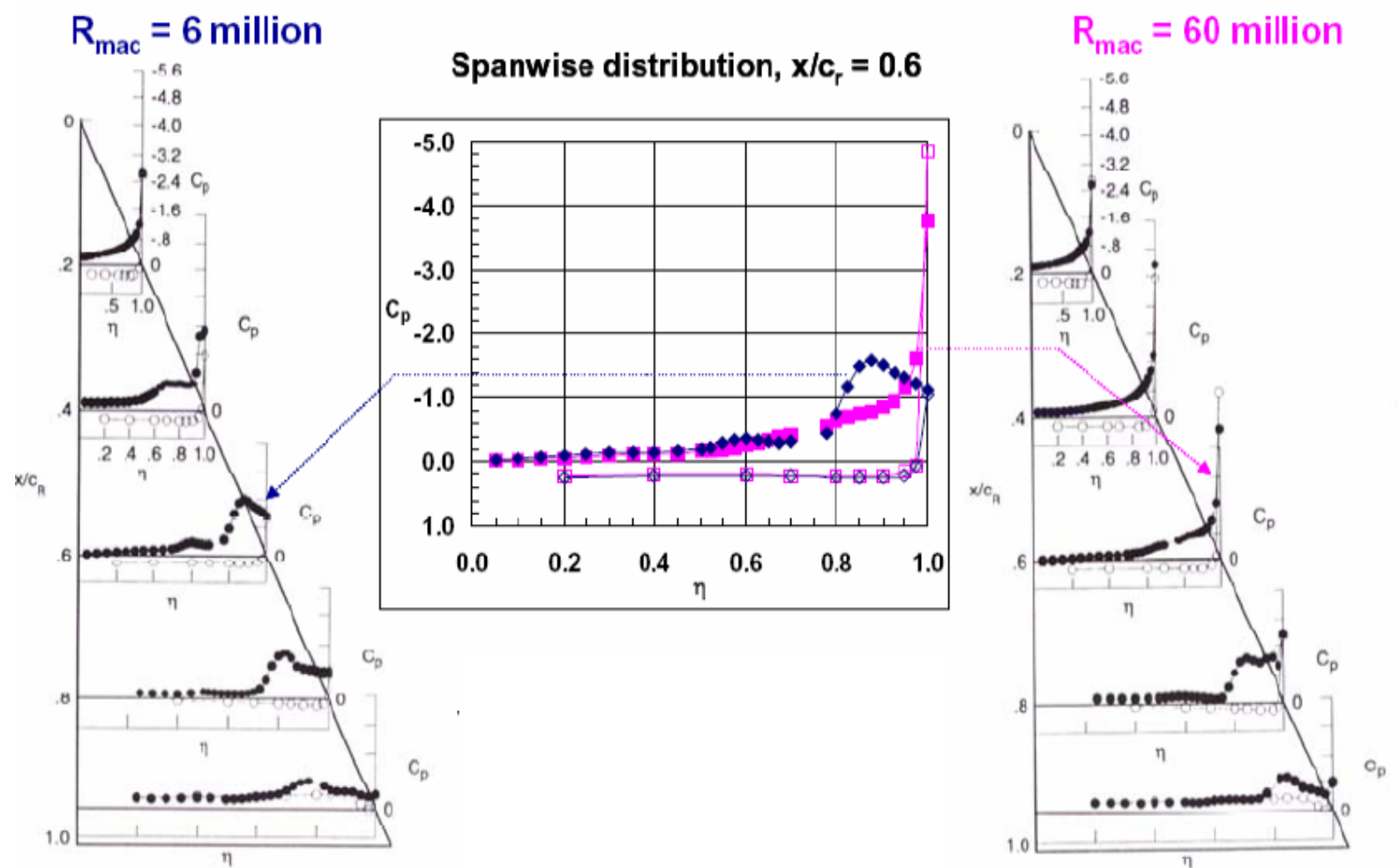

Figure 27. Reynolds number effect, medium leading-edge radius. $M=0.4, \alpha=13^{0}$. Luckring $^{24}$ [2002].
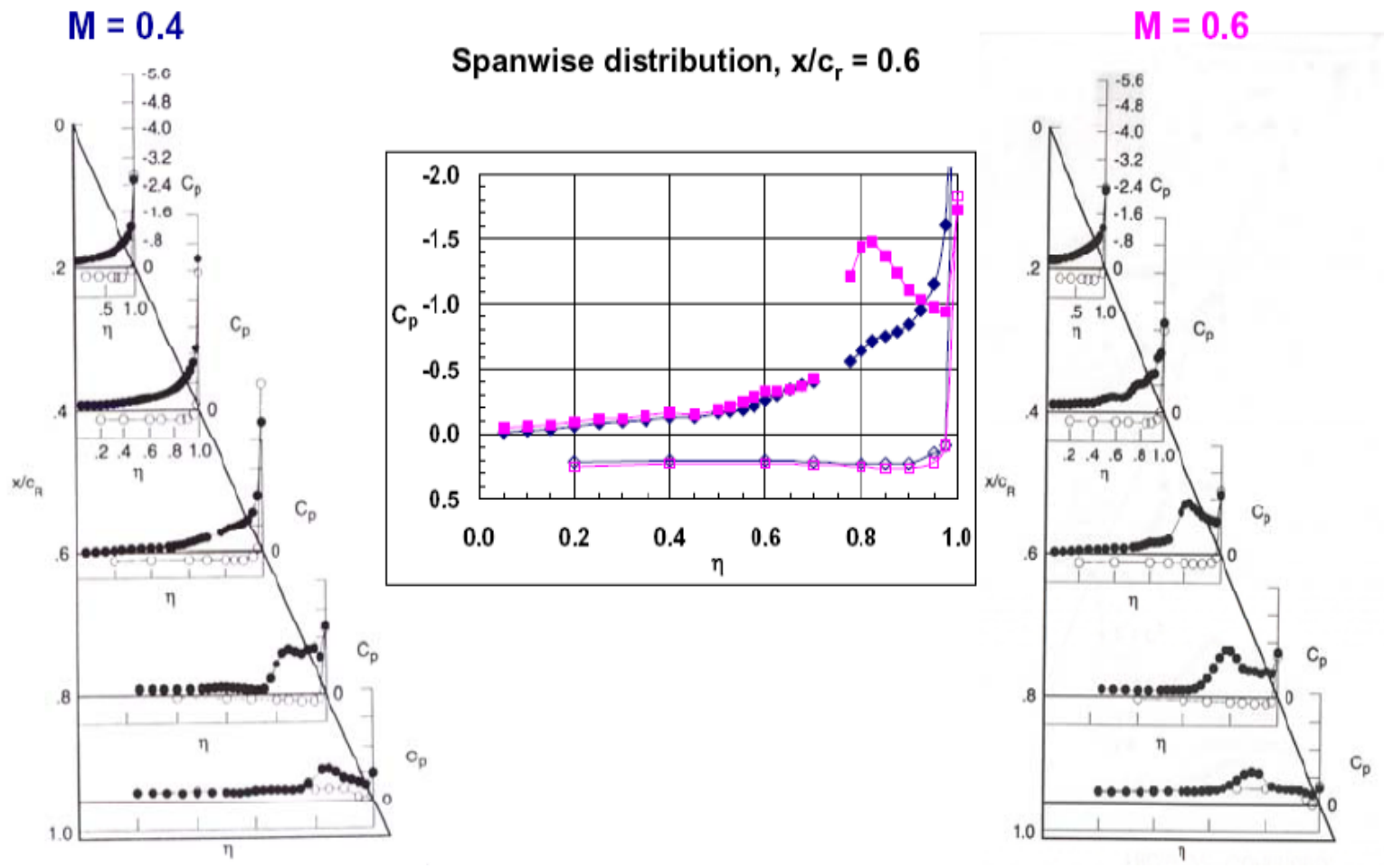

Figure 28. Compressibility effect, medium leading-edge radius. $R_{\operatorname{mac}}=60$ million, $\alpha=13^{0}$. Luckring ${ }^{7}$ [2004]. 
Chicago, IL

These effects of Mach and Reynolds number were generally exhibited by the wings with different leading-edge radii. Analysis of the wing pressures allowed for a means to estimate the onset and progression of the blunt leadingedge separation, and an example for the three blunt wings is shown in Figure 29. The symbols show the angle of attack and location along the leading edge that separation was inferred from the pressure data. There is some uncertainty in this inference, and yet the data provide an indication of the progression of separation and show the delay in this effect with increase in leading-edge radius. The delay in separation due to Reynolds number is also shown.

$$
R_{\text {mac }}=6 \text { million }
$$

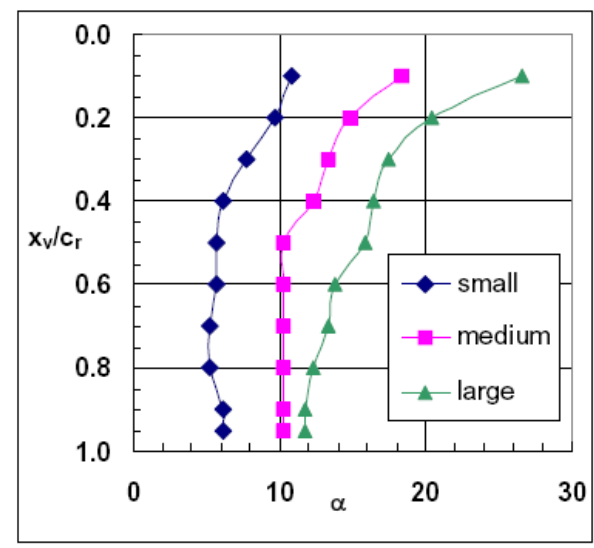

$R_{\text {mac }}=60$ million

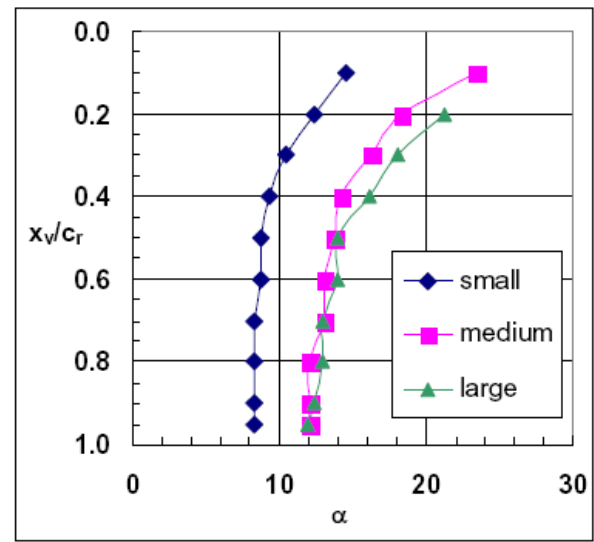

Figure 29. Leading-edge radius effects for the onset and progression of leading-edge separation at low and high Reynolds numbers. $M=0.4$. Luckring ${ }^{24}[2002]$.

A summary of Mach and Reynolds number effects for the onset and progression of the blunt leading-edge separation is shown in Figure 30 for the medium leadingedge radius. The results show the opposite effects of Mach and Reynolds number as regards the blunt leading separation. The contrary effects are consistent with the Mach and Reynolds number effects discussed earlier for airfoil leading-edge separation. In this case the effects are comparable in

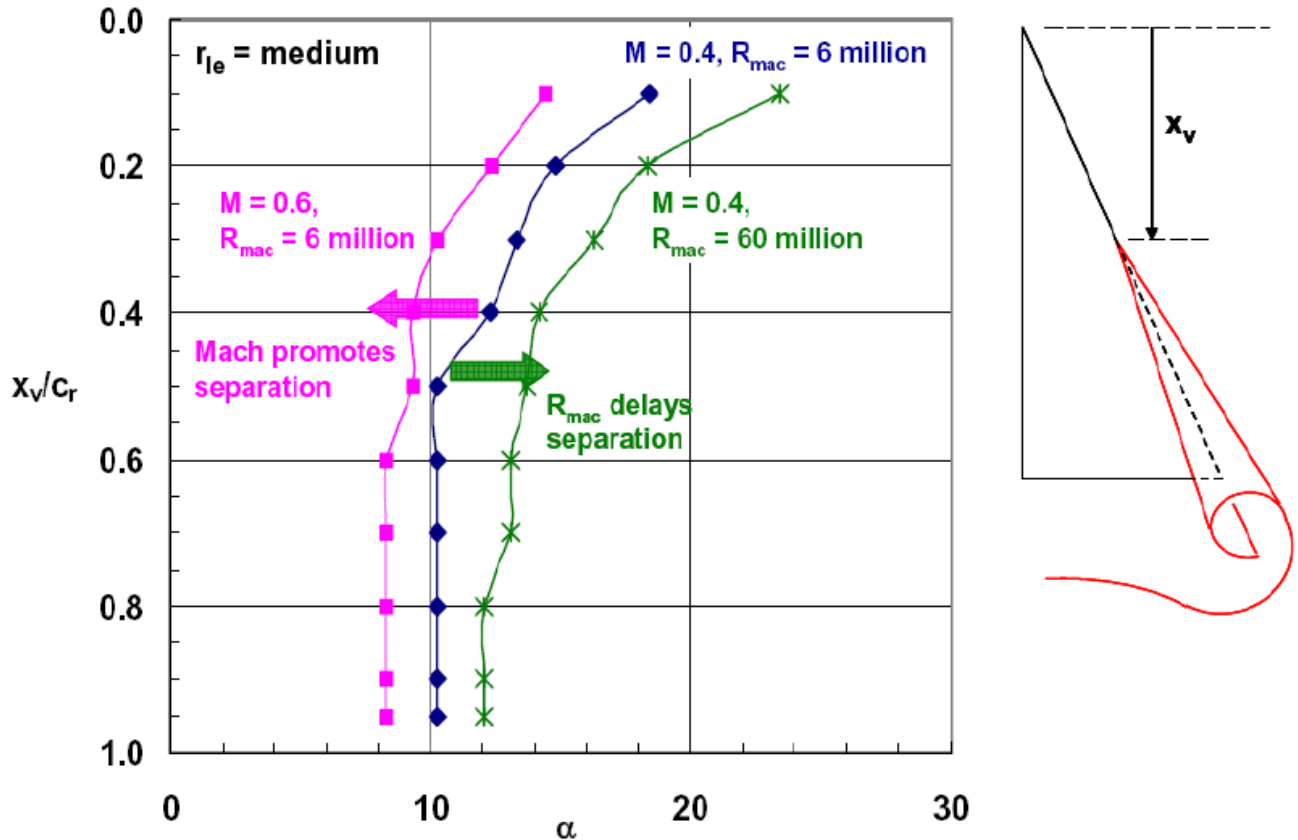

Figure 30. Mach and Reynolds number effects for the onset and progression of leadingedge separation. Luckring ${ }^{26}$ [2004].

magnitude but opposite in sign and require at least a pressure tunnel to be isolated experimentally. 


\section{Recent Research Activities}

Four recent research programs are summarized in this section that have been organized and executed through The Technical Cooperation Program (TTCP) or the Research and Technology Organization (RTO, formerly known as the Advisory Group for Aerospace Research and Development, AGARD).

The TTCP facilitates cooperative research programs among the member countries Australia, Canada, New Zealand, the United Kingdom, and the United States. The TTCP collaborative research activity chosen for this summary was coordinated through the Aerospace Systems Group (AER) and executed by Technical Panel 5 (Fixed Wing System Aerodynamics), collectively known as AER-TP5. This project was focused on aerodynamics of a representative UCAV configuration known as 1303. The activity included both experimental and numerical investigations and was completed several years ago.

The RTO facilitates scientific and technical collaborative activities that are of mutual interest among member nations of the North Atlantic Treaty Organization (NATO). These activities can take a number of forms that include, but are not limited to, symposia, workshops, specialist meetings, and task group programs. Among these, the task groups encompass collaborative research activities. The RTO collaborative research activities chosen for this summary have all been sponsored by the Applied Vehicle Technology (AVT) Panel of the RTO. Although the AVT panel embraces work pertinent to air, land and sea vehicles, those chosen for this paper only address air and sea vehicles, and a further emphasis will be applied to the air vehicle work.

The first RTO activity, identified as AVT-113, has recently concluded and was focused on aerodynamics of the F16-XL-1 aircraft and a delta wing. The second RTO activity, identified as AVT-161, is presently underway and is focused on unsteady aerodynamics of a UCAV concept, the

Table 2. Research configuration wing parameters.

\begin{tabular}{|l|c|c|c|}
\hline Configuration & Sweep, deg & $\begin{array}{c}\text { Aspect } \\
\text { Ratio }\end{array}$ & r/cref, \% \\
\hline 1303 & 47 & 3.9 & $0.4-0.2$ \\
\hline Delta wing & 65 & 1.9 & $0.05,0.15,0.30$ \\
\hline SACCON & 53 & 3.1 & $0.23-0.023$ \\
\hline Diamond wing & 53 & 2.2 & TBD \\
\hline
\end{tabular}
X-31 aircraft, and several naval vessels. The third RTO activity, identified as AVT-183, has recently been initiated and is focused on smooth-surface separation aero/hydro-dynamics for a diamond-wing configuration and a naval surface combatant. Summary wing parameters for the research configurations are listed in Table 2. Parameters for the F16-XL-1 and the X-31 may be found in Table 1.

\section{A. 1303}

The UCAV configuration known as 1303 was originally developed by the Boeing Phantom Works under contract to the Air Vehicles Directorate of the Air Force Research Laboratory (AFRL). The vehicle design was developed to meet certain mission requirements and evolved not only through preliminary design but also a degree of advanced vehicle design to incorporate many features of a practical aircraft. The resultant configuration was a lambda-type blended wing body with a leading-edge sweep of $47^{\circ}$ and trailing edge sweeps of $+/-30^{\circ}$. Original experiments were conducted in the United States, and summary information for the configuration has been given by Billman ${ }^{28}[1998]$.

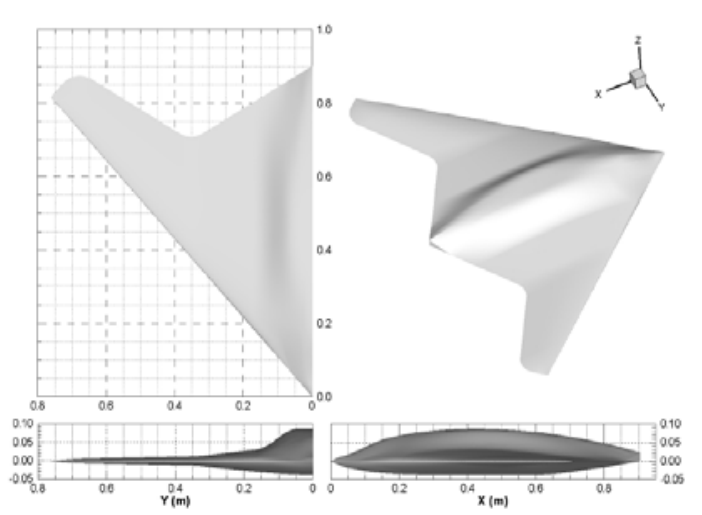

a) 3-view

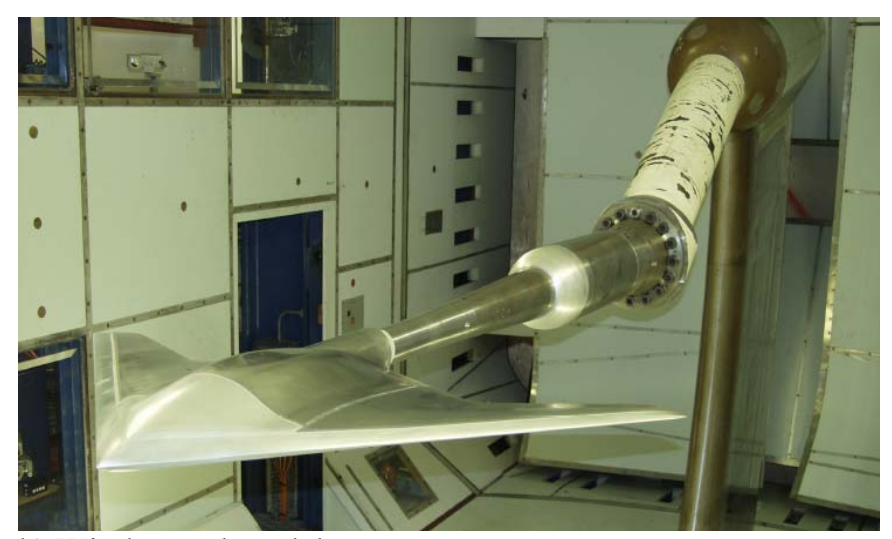

b) Wind tunnel model

Figure 31. Configuration 1303. Petterson ${ }^{39}$ [2006].

This work was conducted by AER-TP5 between 2004 and 2007 and extended the original data sets by adding new measurements and computations for leading-edge radius and Reynolds number effects. A separate model was 
Chicago, IL

fabricated to support new experiments in the QinetiQ 5-m tunnel (Farnborough) for the AER-TP5 project. In addition, an extensive CFD campaign was conducted, and results were reported collectively in a special session at the AIAA $24^{\text {th }}$ Applied Aerodynamics Conference (references 29-36). The model was also used to obtain high-speed data at the ARA 9x8-foot tunnel (Bedford), and data from the two experiments have been reported by Bruce [2003 ${ }^{37}$, $\left.2003^{38}\right]$.

A three-view rendering of the configuration as well as a photograph of the model is shown in Figure 31. The baseline configuration was uncambered with NACA 64A thickness airfoil sections. Thickness varied from 12 percent to 10 percent inboard and the wing (from the inboard crank in the trailing edge) was $8 \%$ thick. The program included assessments for two additional leading edges, sharp and blunt (1.0\% local chord). For comparison, the 64A thickness profiles would have leading edge radii varying from $1.0 \%(12 \% \mathrm{t} / \mathrm{c})$ to $0.4 \%(8 \% \mathrm{t} / \mathrm{c})$.

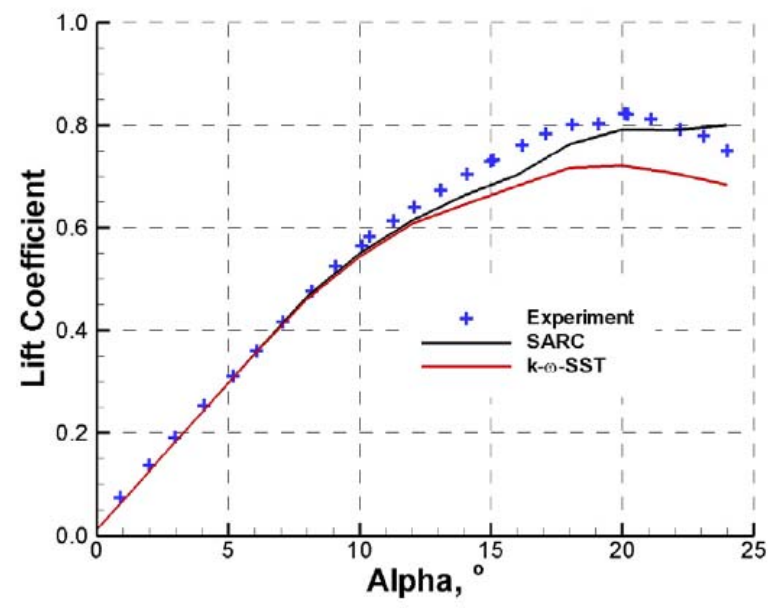

a) Lift

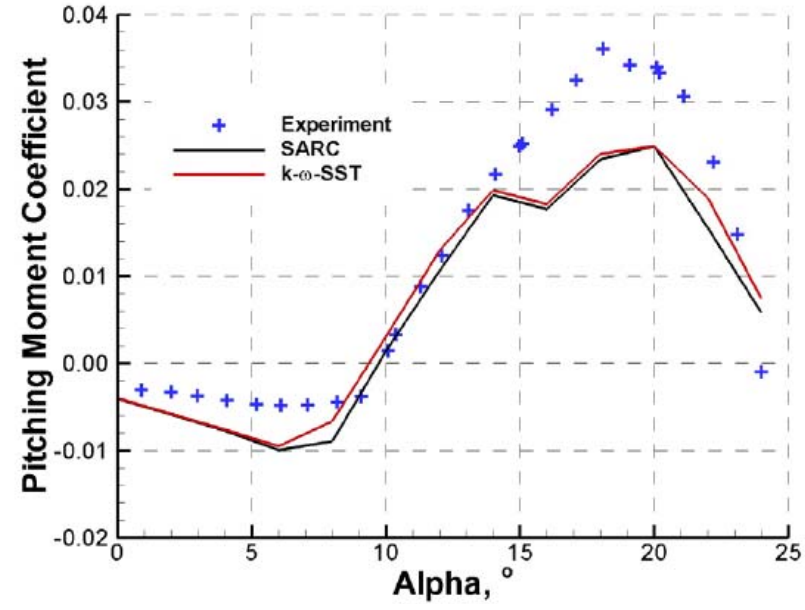

b) Pitching moment

Figure 32. 1303 force and moment characteristics. $M=0.25, R_{\text {mac }}=8.5$ milion. Petterson ${ }^{39}[2006]$.

Tests in QinetiQ 5-meter tunnel at Farnborough were conducted for Mach numbers ranging from 0.13 to 0.30 and Reynolds numbers, based on the mean aerodynamic chord, ranging from 3.1 million to 8.5 million. The test program was designed to isolate Mach and Reynolds number effects utilizing the pressure capability of the 5-m tunnel, and all measurements were performed with free transition. A single common condition between the Mach and Reynolds number transects occurred at a Mach number of 0.25 and a Reynolds number of 4.3 million. Data were obtained between -5 and 25 degrees angle of attack.

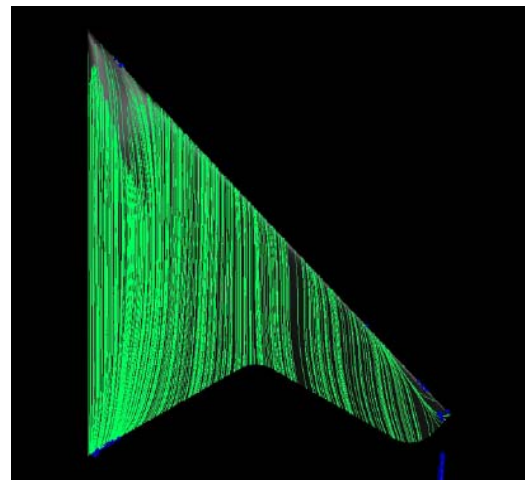

a) $\alpha=4^{0}$

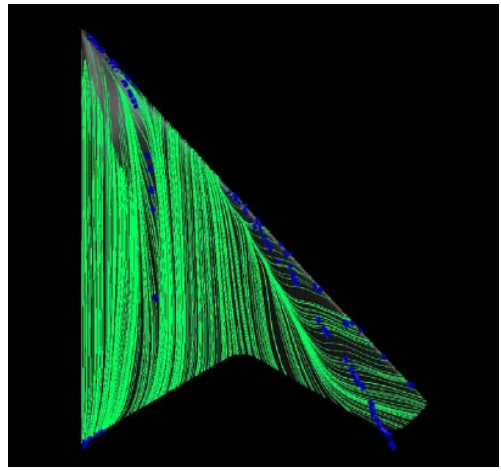

b) $\alpha=\mathbf{8}^{0}$

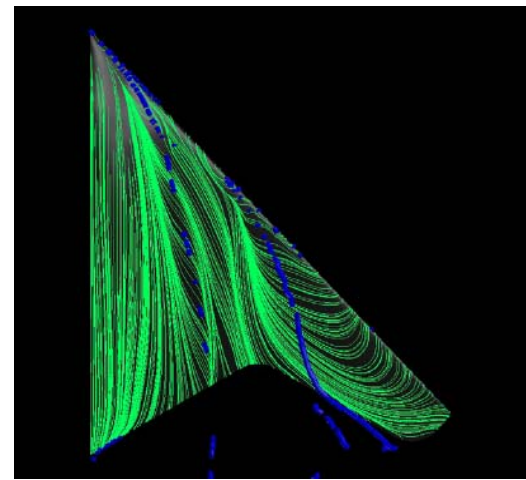

c) $\alpha=12^{\circ}$

Figure 33. Predicted 1303 progression of leading-edge separation. $M=0.25, R_{\mathrm{mac}}=8.5$ million. $K-\omega$. Petterson $^{39}$ [2006].

A summary of the low-speed data has been given by McParlin ${ }^{30}$ [2006] and precursor CFD was published by Petterson $^{39}$ [2006]. The results document the onset and progression of the blunt leading-edge vortex separation and demonstrate an adverse pitch-up associated with this flow. Sample force and moment results are shown in Figure 32 from Petterson ${ }^{39}$ [2006], and the corresponding surface flow patterns from Petterson's computations are shown in Figure 33. Attached flow was achieved at low angles. Sectional loading will be a maximum at the station corresponding to the trailing-edge break, and by 8 degrees angle of attack the origin of the leading-edge vortex appears to be near this station and the pitch-up process is underway. (CFD showed separation at 6 degrees for this 
station as well). The experimental pitch break was fairly well approximated by CFD and the pitch-up magnitude beyond this break was well captured by the computation. The inboard flow may have a very small vortex coming from the wing apex at 8 degrees angle of attack. At 12 degrees there appears to be more evidence of the inner vortex but it still remains separated spanwise from the outer leading-edge vortex. It should be recalled that the 1303 vehicle concept is not a full aircraft design, but moment nonlinearities can occur near the boundaries in intended operation and can present a challenge to the flight mechanics. In that regard it would be useful to be able to predict these phenomena.

Initial analysis of transition consequences to this flow was reported by Arthur ${ }^{40}$ [2006]. A coupled RANS/boundary-layer/ $\mathrm{e}^{\mathrm{N}}$ numerical method was applied to several geometries that included a single 1303 condition. The natural transition results showed altered flow patterns in the insipient separation and subsequent part-span vortex flow, as compared to fully turbulent computations, and the transitional computations agreed better with experiment. Subsequent publications addressed further modeling considerations and numerical effects (e.g., grid resolution) with application to six additional 1303 test conditions [Arthur ${ }^{41}$, 2007] as well as a more advanced computational approach based upon coupling RANS and three-dimensional boundary layer methods [Arthur ${ }^{42}$, 2009] with application to the 1303 configuration at a very low angle of attack.

\section{B. AVT-113}

Task Group AVT-113 was entitled "Understanding and Modeling Vortical Flows to Improve the Technology Readiness Level for Military Aircraft” and conducted its activities from January of 2004 to May of 2008. Summary findings of this Task Group have been recently published by the $\mathrm{RTO}^{43}$ [2009]. The Task Group had two sub groups, commonly referred to as facets. The first facet was focused on CFD predictions of the F-16XL-1 aircraft and the second facet was focused on experimental measurements and CFD assessments of certain delta wing aerodynamics. These two facets are summarized below as regards blunt leading-edge separation aerodynamics.

\section{F-16XL-1 Facet}

A unique flight test data set had been developed through collaboration between the NASA Dryden and NASA Langley Research Centers and published by Lamar $^{44}$ [2001]. The activity, known as the Cranked Arrow Wing Aerodynamics Project (CAWAP), provided a wealth of flight-test flow field details including wing surface pressures, boundary-layer profiles, surface skin friction, and surface flow visualization for the F-16XL-1 aircraft, Figure 34. A summary of this activity was given by $\operatorname{Lamar}^{45}$ [2003] at the RTO symposium

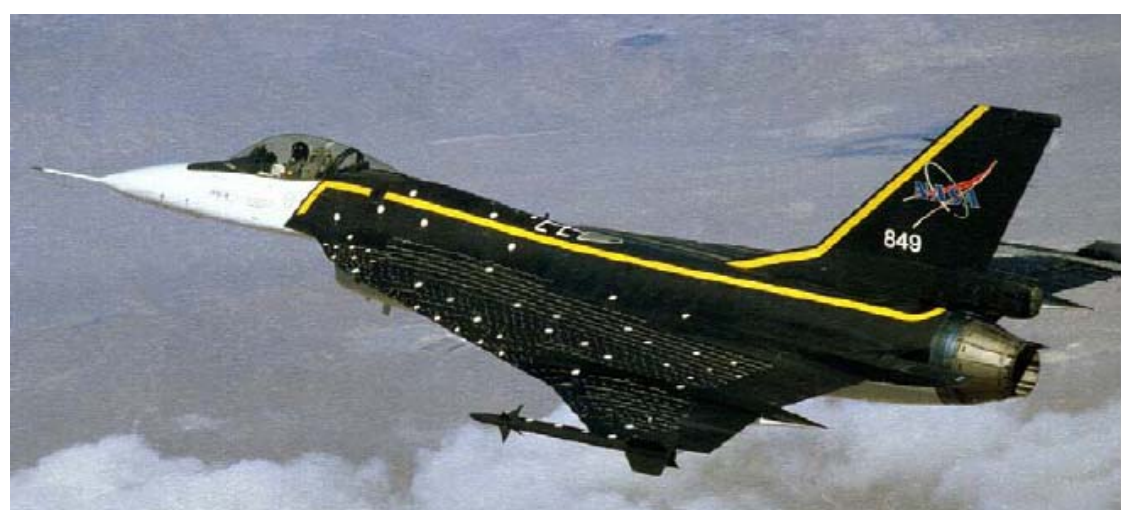

Figure 34. F-16XL-1 aircraft. on Advanced Flow Management, Part A - Vortex Flows and High Angle of Attack for Military Vehicles ${ }^{46}$ [2003] which convened in 2001. This summary served as the genesis of what became the Cranked Arrow Wing Aerodynamics Project International (CAWAPI) embodied in this facet of AVT-113.

This facet focused strictly on CFD assessment of the already available flight test data for the F-16XL-1 aircraft. Seven flight test conditions were chosen at zero sideslip that nominally span Mach numbers from 0.30 to 0.97, Reynolds numbers (based on an aircraft reference chord of 24.7 feet) from 32 million to 89 million, and angles of attack from 4 to 20 degrees. Several sideslip cases were also included. CFD solutions were contributed from nine groups using ten different solvers, including both structured and unstructured approaches, to assess predictive capabilities for these data. Two special sessions at the $45^{\text {th }}$ AIAA Aerospace Sciences meeting highlighted these individual contributions [references 47-57], and a summary paper by Rizzi ${ }^{57}$ [2007] was also included.

Considerable effort went toward modeling the complex aircraft geometry at the high Reynolds numbers of the flight test data. Resulting grids were relatively large (most had around 10 to 30 million points) at the time of the work. From the perspective of this paper, it is noted that the complex aircraft modeling included representation of the small but blunt leading edge of the $70^{\circ}$-swept portion of the wing. (The outboard $50^{\circ}$-swept portion of the wing has a sharp leading edge). 
A representative subsonic solution was reported by Rizzi ${ }^{57}$ [2007], based upon work from Boelens ${ }^{47}$ [2007], and is shown in Figure 35. A good resolution of this vortical flow was achieved, and correlations between the collective computations and flight test data at subsonic conditions were generally good. Transonic cases at lower angles of attack proved to be more difficult with speculation that shock/vortex interactions were not being adequately captured [Rizzi $\left.{ }^{57}, 2007\right]$.

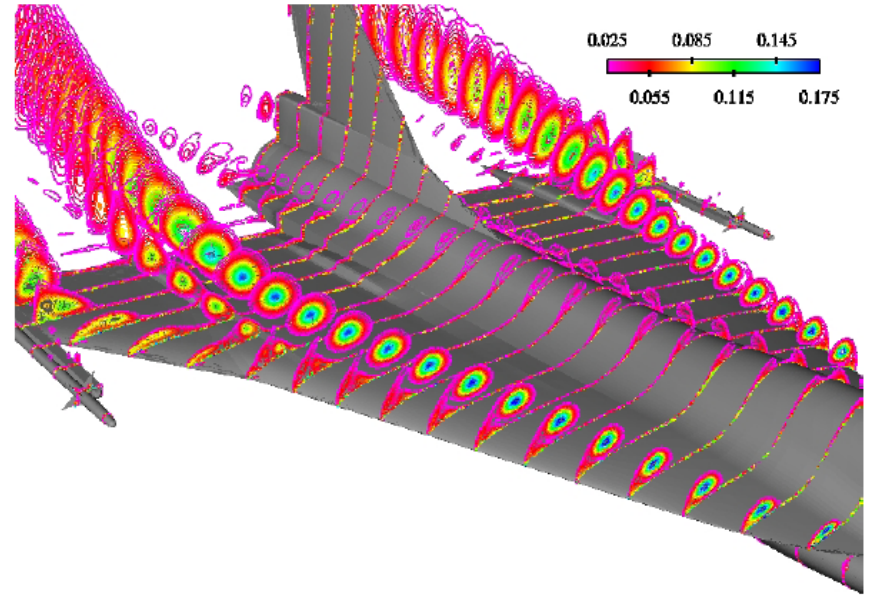

a) Total pressure contours

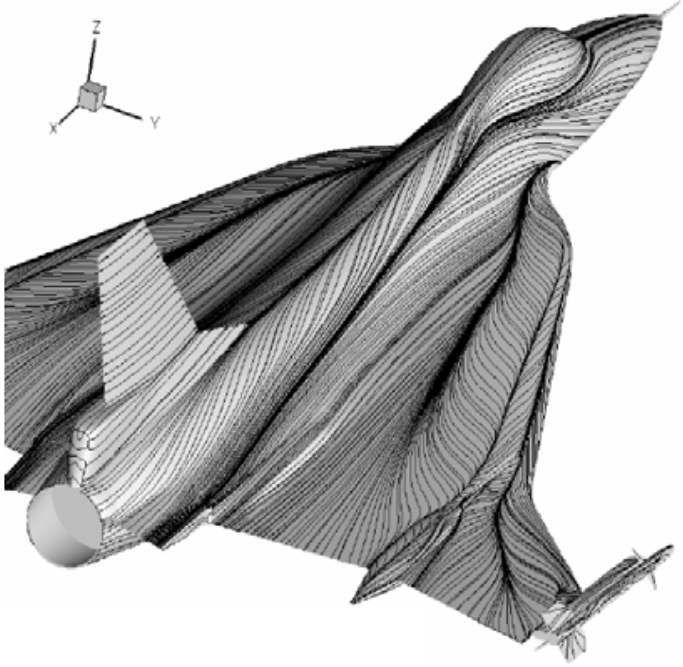

b) Skin friction

Figure 35. F-16XL-1 computational results. $M=0.304, R_{\text {cref }}=44$ million, $\alpha=11.9^{\circ}$. Rizzi $^{57}$ [2007], Boelens ${ }^{47}[2007]$.

The CAWAPI results have demonstrated that good correlations can be achieved for a complex slender-wing aircraft, with blunt leading edge representation, at some flight conditions and this is a significant accomplishment. However, much work remains to be done at this level of complex aircraft modeling to assess factors such as separation onset and progression, leading-edge radius effects, Reynolds number effects, shock/vortex interactions, and so forth. It is also possible to address aspects of these factors through reduced-complexity or unit problem studies, and this was done as part of the other facet in AVT-113.

\section{Delta Wing Facet - Vortex Flow Experiment 2}

This facet had its origins in the NTF delta wing data discussed in Section IV above, and some early reporting of these results by Luckring ${ }^{58}$ [2001]. Subsequent discussions led to a proposal for a new vortex flow experiment in a paper by Hummel $^{59}$ [2003] which was also presented in 2001 at the RTO symposium on Advanced Flow Management, Part A - Vortex Flows and High Angle of Attack for Military Vehicles ${ }^{46}$ [2003]. This paper served as the genesis of what became known as Vortex Flow Experiment 2 (VFE2) embodied in this facet of AVT-113.

This facet was primarily focused on developing an understanding of the curious inner and outer co-rotating vortices inferred from analysis the blunt leading-edge data from NTF. It was clear that additional and detailed experimentation would be required to provide insight into this particular vortical flow, and that a parallel CFD modeling study would be of considerable interest. Because the delta wing geometry was so simple (and analytical), detailed numerical modeling studies would be more feasible than what could be achieved with the complex CAWAPI aircraft configuration. In addition, leading-edge radius effects as well as Mach number and Reynolds number effects were already in hand from the prior NTF experiments.

Experimentation was partially facilitated by sending the $3 / 4$-scale model tested in the NASA LaRC LTPT to Europe. Four additional models were fabricated among participating organizations from Germany, France, and Scotland primarily to facilitate instrumentation and for facility considerations. New experimental results were contributed by five organizations using six facilities thus providing a means for multiple models, multiple facilities, and multiple instrumentation suites to be used to understand this flow. CFD modeling assessments were contributed by seven organizations using seven codes, including both structured and unstructured approaches, to assess and develop a predictive capability for this flow. Two special sessions at the $46^{\text {th }}$ AIAA Aerospace Sciences meeting 
Chicago, IL

highlighted these individual contributions [references 60-74]. These sessions included both an experimental summary paper by Luckring and Hummel $^{66}$ [2008] as well as a computational summary paper by Fritz ${ }^{74}$ [2008].

Experimental results by Konrath ${ }^{62}$ [2008] confirmed the existence of the dual co-rotating leading-edge vortices, replicated conditions of this flow as observed from the NTF data, and provided considerably more detail of the flow from the use of pressure sensitive paints. This was achieved with the LTPT model in the DLR Göttingen Transonic Wind tunnel (TWG). An example is shown in Figure 36. The detailed differences between left and right semispan
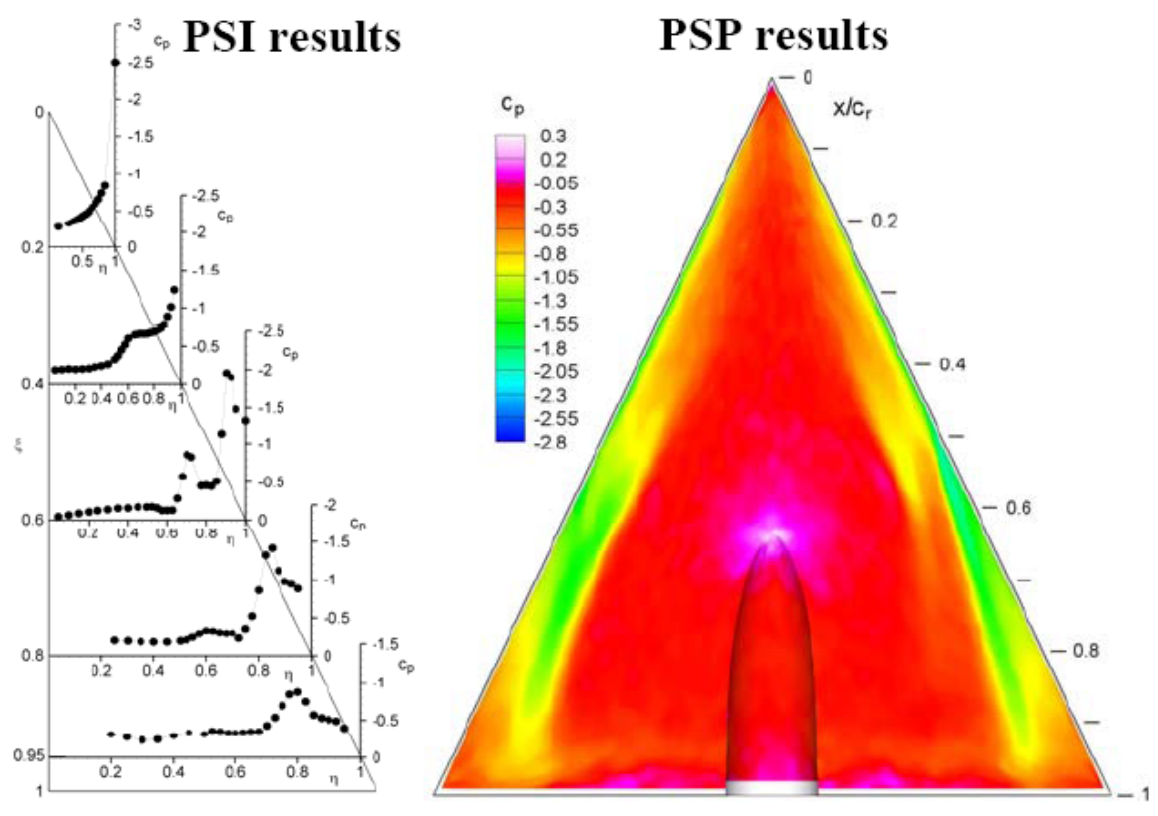

PSP results

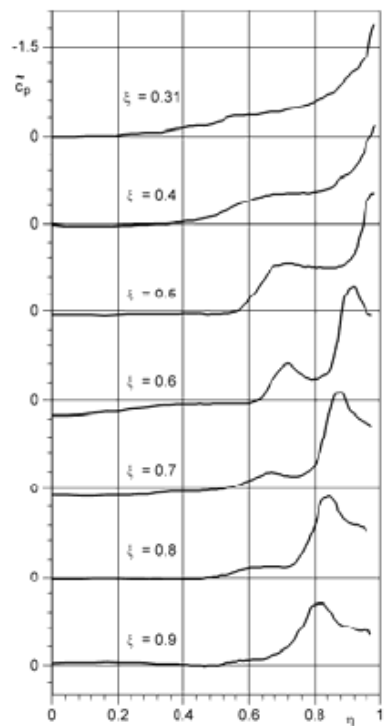

Figure 36. Pressure sensitive paint measurements. $M=0.4, R_{\mathrm{mac}}=3 \cdot 10^{6}, \alpha=13^{\circ}$. Konrath $^{62}[2008]$.

patterns could be due to small differences in the model geometry or due to small alignment differences between the model and free stream flow. Flow physics measurements were obtained at the Technical University Munich (TUM), and an example of this work from Furman ${ }^{64}$ [2008] is shown in Figure 37. Here mean and fluctuation flow field measurements, as well as fluctuating surface pressure, are displayed for several locations on the delta wing. These types of measurements should aid with the assessment of various viscous flow simulation models for predictions of this flow. Some consideration to boundary layer states and transition was given by Hummel ${ }^{75}$ [2004], and some aspects were addressed in the experiments by Furman $^{64}$ [2008].

Six primary conditions were identified for CFD assessments as summarized by Fritz ${ }^{74}$ [2008]. Emphasis was retained on the part-span blunt leading-edge separation at $13^{\circ}$ angle of attack. Approximately 21 additional cases of interest were also identified for potential study. The other conditions allowed for assessments between sharp and blunt leading edges, unburst and burst leading-edge vortices, and transonic shock-vortex interactions. Grids typically ranged from 2 to 26 million points. Among the additional cases, the transonic CFD studies of Shiavetta ${ }^{70}$ [2008] are noteworthy.

One of the better comparisons for the part-span vortex case is presented in Figure 38 between stereo PIV and PSP measurements of Konrath ${ }^{62}$ [2008] and the numerical results of Fritz ${ }^{68}$ [2008]. The overall structure of the dual inner and outer vortices appears to have been fairly well simulated in this comparison. Other methods also showed considerable progress toward simulating these flows, although the prediction of the separation onset position remained a challenging aspect for CFD. Experimental details of the flow field leading up to insipient separation were not obtained to the same extent as for the rest of the vortical flow, and was felt that such details would further aide CFD method improvements. This point will be further addressed in the section regarding AVT-183. 


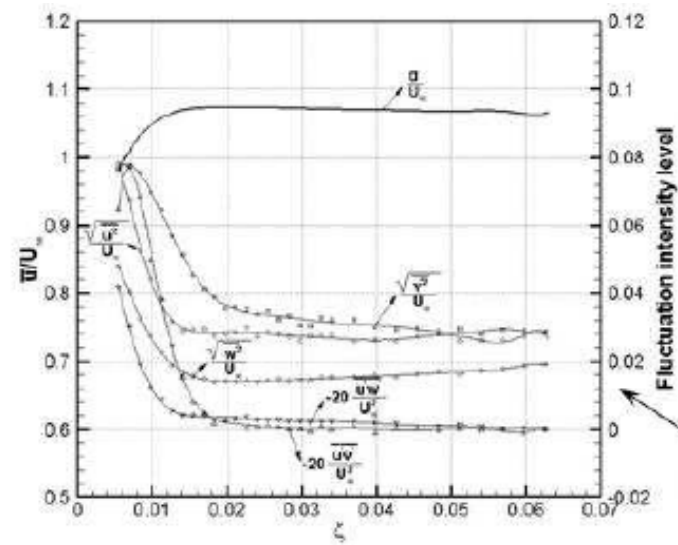

a) $x / c_{r}=0.4, \eta=0.4$
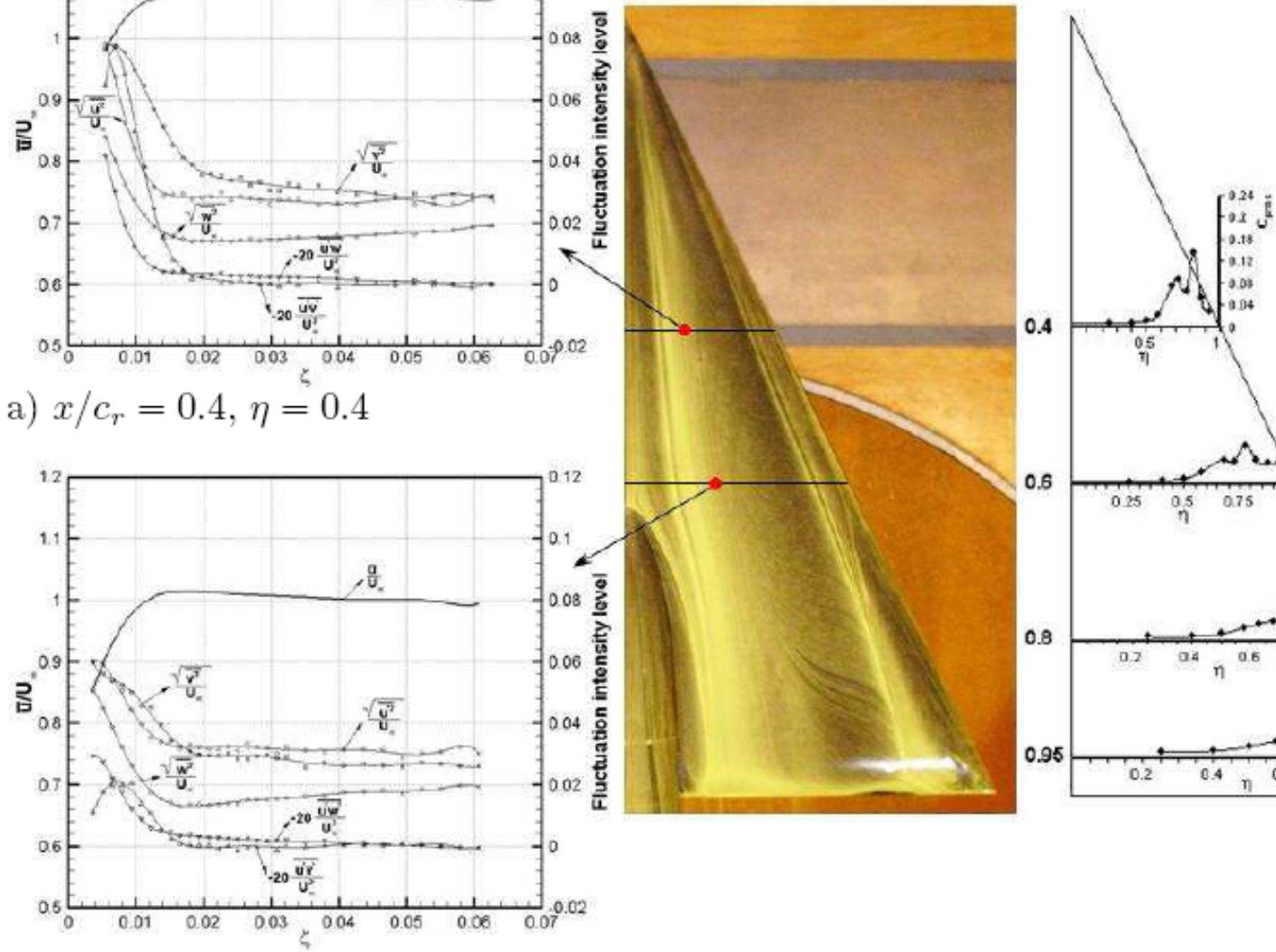

b) $x / c_{r}=0.6, \eta=0.4$

Figure 37. Flow physics measurements. $M=0.07, R_{\operatorname{mac}}=1 \cdot 10^{6}, \alpha=18^{\circ}$, medium leading-edge radius. Furman $^{64}[2008]$.

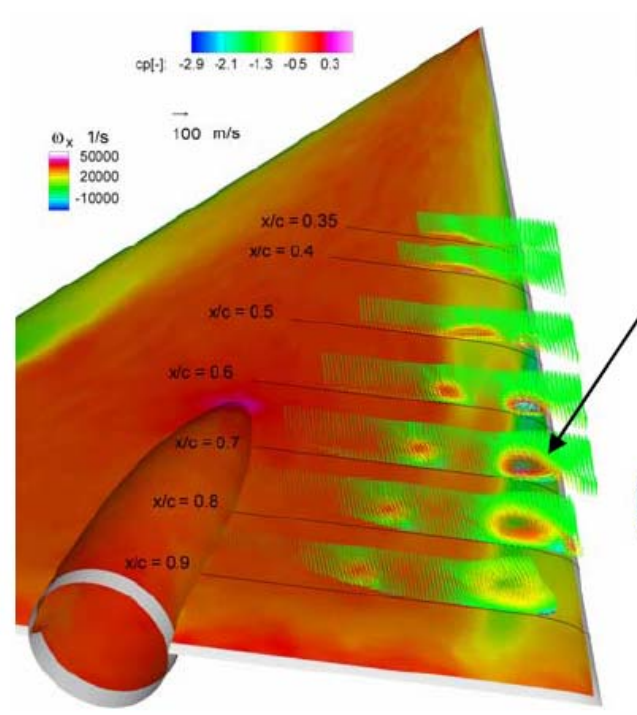

Experiment

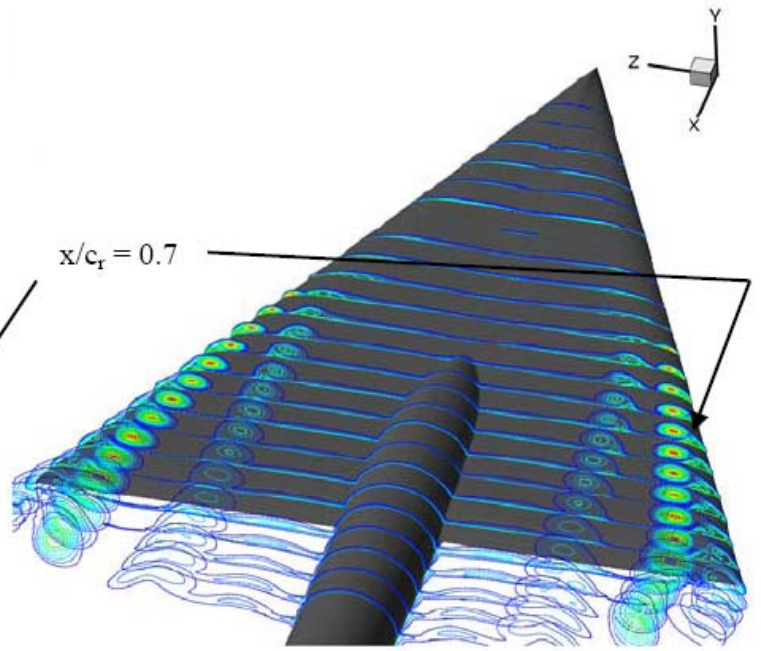

Numerical solution

Figure 38. Flow field measurements and predictions. $M=0.4, R_{\text {mac }}=3 \cdot 10^{6}, \alpha=13^{\circ}$, medium leading-edge radius. Konrath ${ }^{76}[2006]$ and Fritz $^{68}[2008]$. 


\section{AVT-161}

Task Group AVT-161 is entitled "Assessment of Stability and Control Prediction Methods for NATO Air \& Sea Vehicles" and is scheduled to conduct its activities from January of 2008 through May of 2011. This task group has three facets, two of which are focused on aircraft applications. One air facet is focused on the X-31 aircraft, whereas the other is focused on a representative UCAV configuration known as SACCON (Stability And Control CONfiguration). The third facet is focused on a naval application. The task group is currently in progress, and a first reporting of their results is contained primarily in two special sessions at this $28^{\text {th }}$ AIAA Applied Aerodynamics Conference [references 77-89].

The task group's orientation to stability and control includes both static and dynamic conditions, and a considerable (perhaps primary) effort is being directed toward obtaining dynamic data and dynamic CFD simulations for the vehicle interests. However, to do the dynamic studies requires baseline information to also be obtained for static conditions. Several aspects of the air facet work regarding blunt leading-edge separation aerodynamics at the static conditions are highlighted below.

\section{1. $X-31$ facet}

The X-31 represents another complex, full-aircraft geometry modeled with CFD. The wing has cranked leading edge with $57^{\circ}$ sweep inboard and $45^{\circ}$ sweep outboard, and an aspect ratio of approximately 2.9. The configuration also incorporated a set of canards, a small strake ahead of the wing, and an aft-body strake as well. These configuration details can be observed in the photograph of the wind tunnel model used as part of the AVT-161 program, Figure 39. In addition, segmented leading-edge flaps can be observed. All of these features represented the fullscale X-31 aircraft configuration

The blunt wing leading edge was modeled with sufficient detail to represent gaps between the leading-edge flap segments, and an assessment of the gap effects in the CFD simulations was performed by Boelens $^{90}$ [2009]. In these assessments the leadingedge flaps were all undeflected. Results at $10^{\circ}$ angle

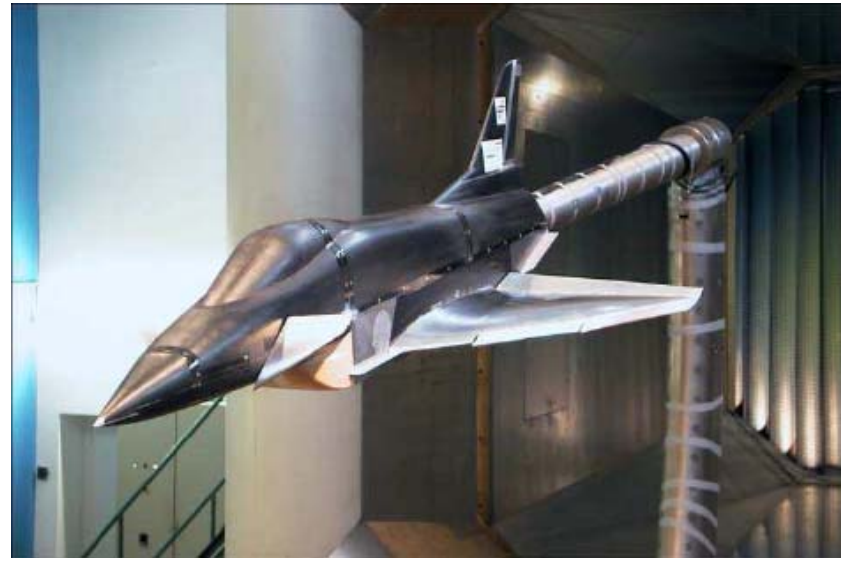

Figure 39. $X-31$ model in DNW low-speed wind tunnel Braunschweig. of attack showed an attached flow about the wing leading edge and most the wing itself. Above $12^{\circ}$ leading-edge vortex separation occurred, but the nature of this separation was significantly affected by the streamwise flap gaps. An example is shown Figure 40 where a cascade of co-rotating leading-edge vortices can be seen outboard over the wing. (Other vortices from the canard and strake can be seen inboard). The origins of these wing vortices are the flap gaps, and Boelens concluded that the presence of the gaps significantly affected the nature of the blunt leading-edge vortical separation. This presents another challenge for modeling onset and

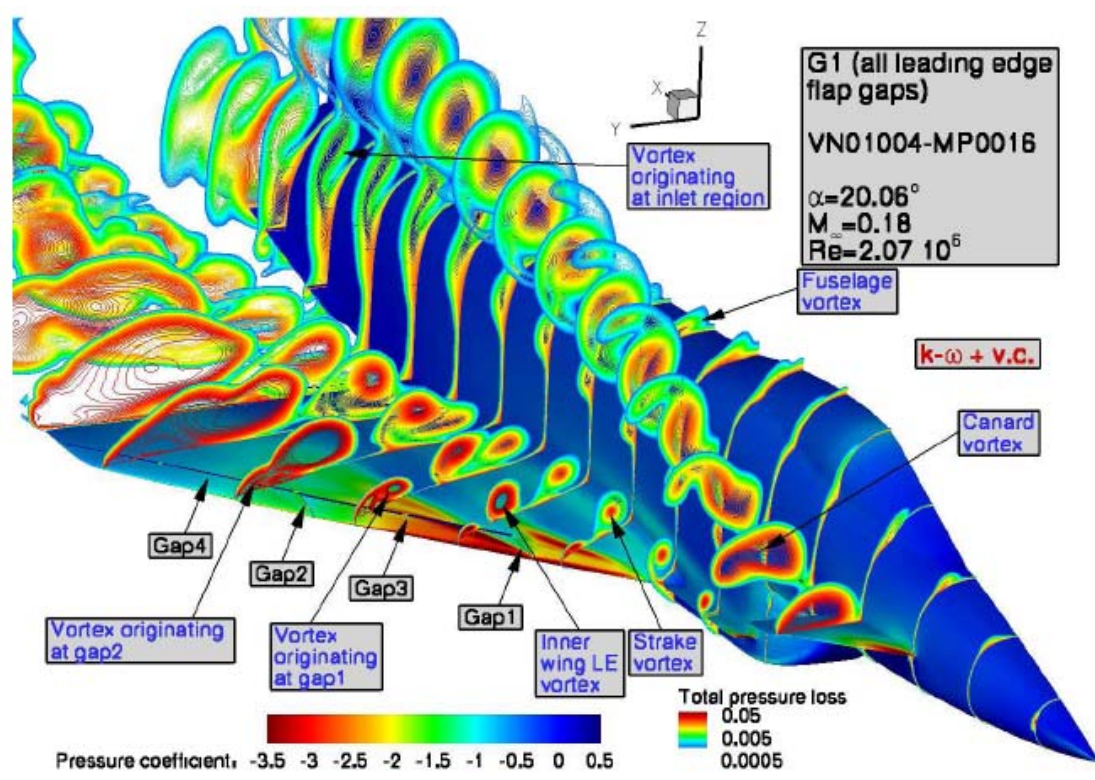

Figure 40. $X-31$ CFD results. $M=0.18, R_{\text {cref }}=2 \times 10^{6}, \alpha=20^{\circ}$. Boelens ${ }^{90}[2009]$. 
Chicago, IL

progression of the leading edge separation.

\section{SACCON facet}

The SACCON configuration was designed by EADS to capture many aspects of UCAV concepts while at the same time being suitable for international collaborative research. The configuration is shown in Figure $\mathbf{4 1}$ and has $53^{\circ}$ swept leading and trailing edges. It falls in the lambdawing class of configurations with a constant chord outer panel and has an aspect ratio of approximately 3.1. The SACCON model has a root chord of approximately 1 meter, and a photograph of the model in the DNW low-speed wind tunnel at Braunschweig is also shown in Figure 41. The model was fabricated to facilitate dynamic stability and control testing.

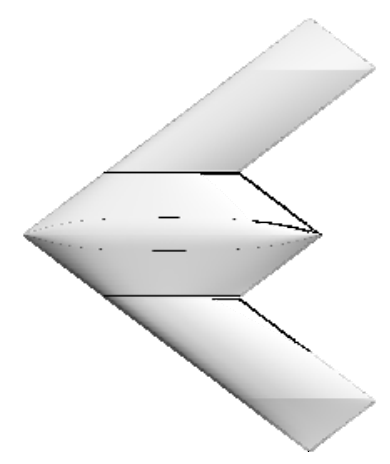

a) Planform.

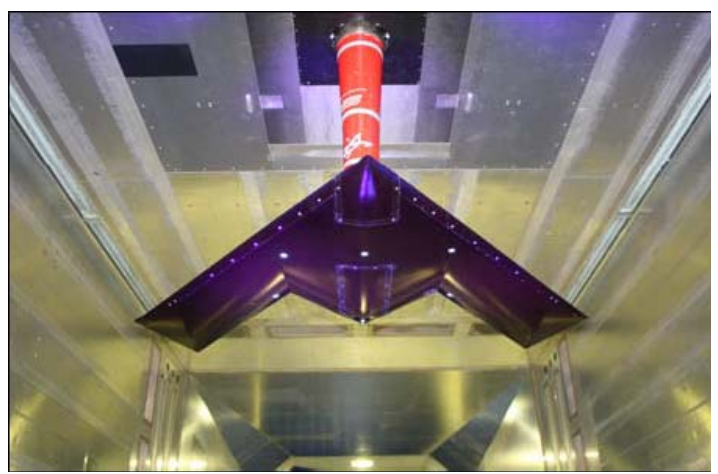

b) Wind tunnel model.

Figure 41. SACCON configuration.

Spanwise distributions of relative thickness (t/c) and leading-edge radius are shown in Figure $\mathbf{4 2}$ along a planform representation. Outboard of the first trailing edge break, these leading-edge radii vary between approximately $0.23 \%$ and $0.023 \%$ of the reference chord. For SACCON, the reference chord was taken as the constant chord of the outer wing panel. The wing design also incorporated outboard twist to delay separation onset effects to higher angles of attack than would have been realized by a planar wing.

Design objectives for low angle of attack attached flow were achieved. The progression of leading-edge separation for this particular geometry results in a complicated vortical flow. An example of this is indicated in Figure $\mathbf{4 3}$ by the CFD results of Frink $^{82}$ [2010]. Results at nominally $5^{\circ}$ angle of attack demonstrate the attached flow. The results at $18^{\circ}$ angle

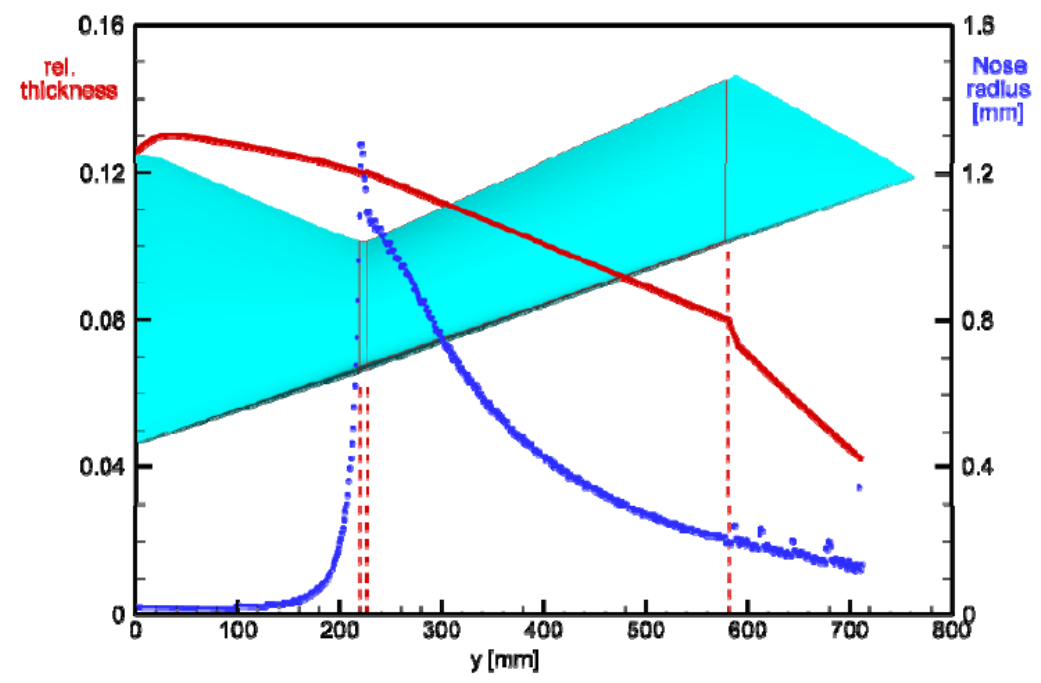

Figure 42. SACCON spanwise thickness and leading-edge radius distributions. of attack were chosen to illustrate some aspects of the complex vortical separation on this configuration. Due to the wing sweep and the particular leadingedge radius distribution, the inner portion of the wing has already developed a primary leading-edge vortex. The leading-edge radius of this portion of the wing is very small, and this inner vortex may share many attributes with a sharp leading-edge vortex. For the outboard portion of the wing, separation onset and progression has occurred such that the origin of the outer primary vortex is adjacent to the inner primary vortex.

Further increases in angle of attack result in vortex interactions between the co-rotating inner and outer primary vortices and have been interpreted as a source of nonlinear force and moment characteristics for this wing by Frink [2010]. The onset and progression of the blunt leading-edge vortical flow from the outer wing establishes the position and strength of the outer vortex and thus this separation is critical to the vortex interaction aerodynamics. 


\section{It should also be noted that the leading-edge vortex characteristics of the SACCON wing are quite different than those of the 1303 wing discussed earlier. The 1303 leading-edge radius design resulted in only a very small apex vortex such that the onset of blunt leading- edge separation from the outer wing progressed into an essentially attached flow on the inner portion of the wing. \\ Although the focus of AVT-161 is on the dynamic stability characteristics of this configuration, it became clear early in the program execution that the static case included some very complex vortex- flow aerodynamics. These developments among others}
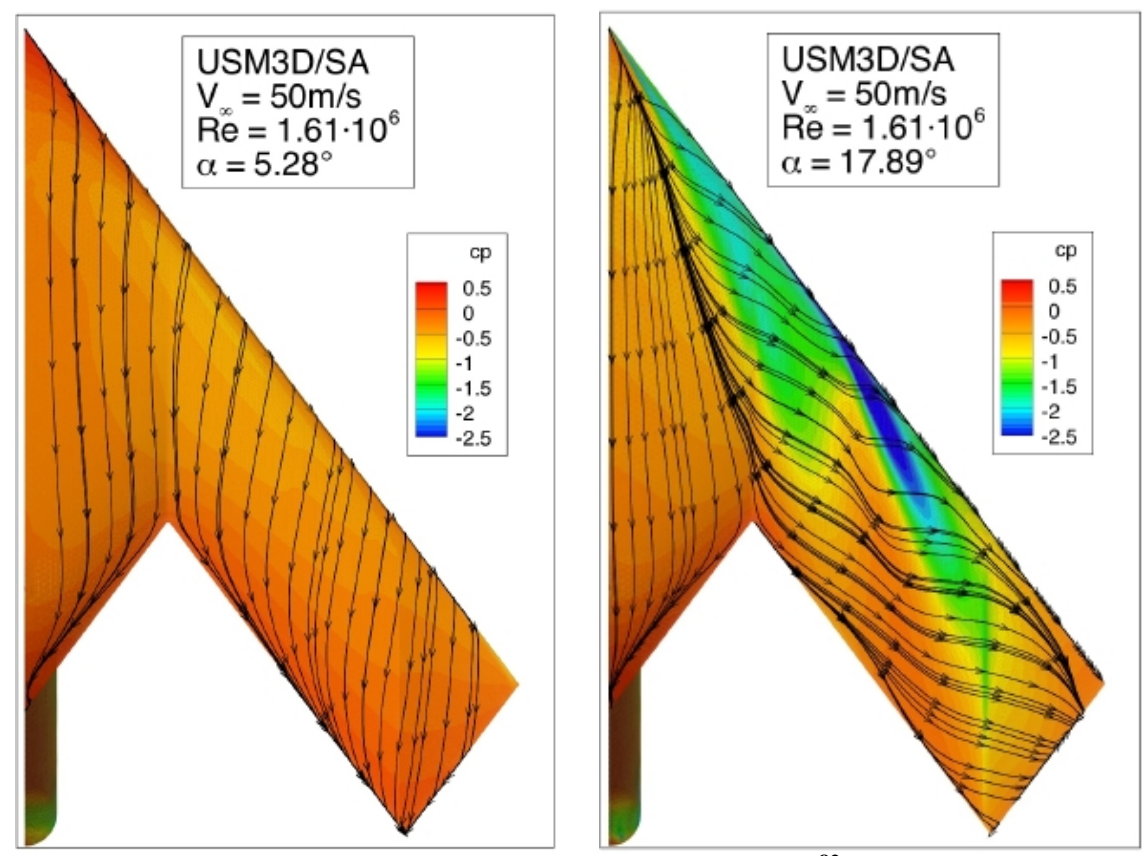

Figure 43. SACCON CFD results. Frink ${ }^{82}$ [2010]. led to what has become the focus for the air facet of a new RTO/AVT task group to be discussed next.

\section{AVT-183}

Task Group AVT-183 is entitled "Reliable Prediction of Separated Flow Onset and Progression for Air and Sea Vehicles" and is scheduled to conduct its activities from January of 2010 through May of 2013. Emphasis is placed on smooth-surface separation, and the task group has an air and a sea facet. The air facet is focused on experimental measurements and CFD assessments of a diamond-wing configuration pertinent to UCAV aerodynamics and the sea facet is focused on experimental measurements and CFD assessments of a naval surface combatant vehicle. Both facets are interested in predicting blunt leading-edge separation pertinent to their vehicle class. Although the work for these facets has only recently commenced, a summary for the program plans is provided below as regards blunt leading-edge separation aero/hydro-dynamics.

\section{Air Facet}

The concept for this facet is to establish a unit problem that is relevant to the AVT-161 SACCON aerodynamics as well as related UCAV concepts. For these semi-slender and swept configurations there is a hierarchy of vortexflow topics to consider. At low speeds and static conditions these would include blunt leading-edge separation onset and progression, co-rotating vortex interactions, vortex breakdown, and vortex hysteresis. Higher speeds would introduce compressibility and vortex-shock interaction effects, and finally dynamics compounds the complication of all the above. Transition is not to be overlooked, and from this collective perspective it was clear that the SACCON configuration represented a very complex suite of vortex flow aerodynamics, even at low speeds.

All of these vortex phenomena relate in one way or another back to the blunt leading edge separation onset and progression. For example, the smooth-surface separation establishes the strength and position of the leading-edge vortex, thereby directly affecting matters such as vortex interactions and vortex breakdown. Transition flow physics also manifest very near the blunt leading edge as discussed earlier in this paper; such effects are absent in the sharpedged case.

The intent for this facet is to study in detail the onset and progression of the blunt leading vortical separation, in a manner that is relevant to the SACCON vehicle, such that an enhanced understanding of the underlying flow physics can be achieved and improved CFD prediction capabilities can be enabled. A SACCON-relevant unit problem has been conceived based upon a simple diamond wing geometry which can establish the part-span vortical separation. (See Figure 44.) Some aspects of the SACCON leading edge are represented, but the overall wing is greatly simplified. 
An experimental and numerical campaign is planned. The detailed measurements achieved in Vortex Flow Experiment 2 were very valuable to understanding the vortical structures of interest to that project, and new experiments are being pursued that can provide such insights into the incipient separation of the AVT-183 part-span vortex. Combined with a numerical campaign that will leverage both the AVT-113 and the AVT-161 experiences, the project will strive to understand why some models work better than others for such flow predictions. As such, the experiments and computations will work toward the current principles established for code validation such as have been summarized by Oberkampf ${ }^{91}$ [2002] and in guidelines from both the AIAA $^{92}$ [1998] and the ASME $\left[2006^{93}, 2009^{94}\right]$.

To the extent that CFD modeling is altered, assessments are being considered in three tiers. (See Figure 45.) The first

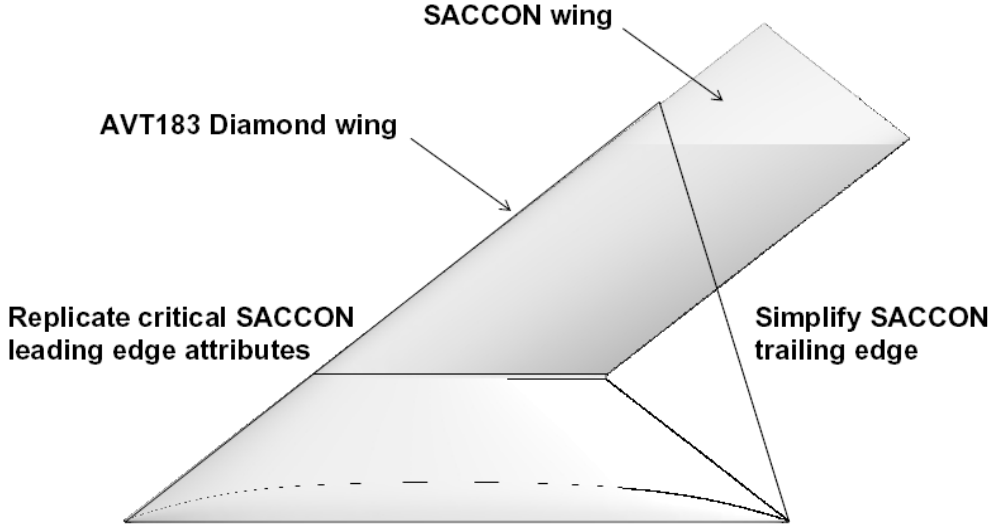

Figure 44. Diamond and SACCON wings.

tier is self-assessment against the data used for the model improvement. Such assessments are necessary for model evaluation, but they are not sufficient for any predictive capability assessment. The second tier constitutes a primary predictive

assessment

where models developed from the diamond wing campaign are applied to the (parent)

SACCON

configuration at relevant conditions to assess predictive improvements.

The third tier constitutes secondary assessments against relevant configurations as shown in the figure and

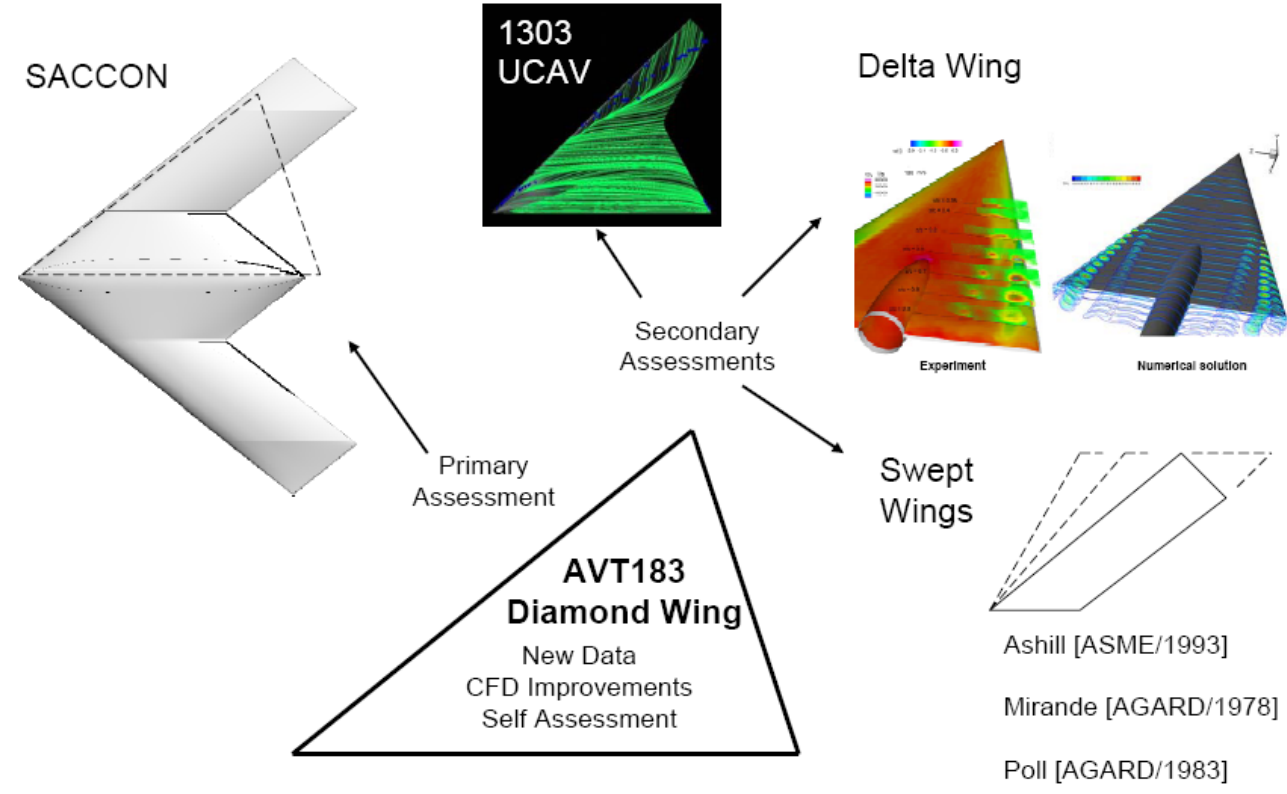

discussed earlier in this paper.

Figure 45. Experimental assessment strategy.

\section{Sea Facet}

The sea facet of AVT-183 is focused on maneuver hydrodynamics that arise on naval surface combatant ships, Figure 46, under high drift and/or yaw conditions. This is another smooth-surface separation that occurs from the bow of the vessel. The work is focused on the David Taylor Model Basin configuration 5415, a representative naval surface combatant hull that incorporates a sonar dome and a transom stern. Sustained experimental and computational activity has been underway to study various flow features with this geometry. This has included periodic workshop assessments, and a lessons-learned summary from a recent workshop has been given by Stern ${ }^{97}$ [2009].

In a manner somewhat similar to the AVT-183 air facet, the sea facet will leverage this prior experimental and numerical work to enhance understanding of why certain numerical methods work better than others and thereby seek improved prediction capability through the project. A combined experimental and computational campaign has 
been initiated that is currently envisioned to include tow-tank experimentation along with computational assessments from several levels of numerical formulations.

Recent computational results by $\operatorname{Stern}^{98}$ [2009] have contrasted URANS and DES simulations of the 5415 flow at high drift angles. Sample results related to this work are show in Figure 47 from Stern $^{99}$ [2008]. Complex vortical structures can be seen in Figure 47a for the separation onset from DES simulations. In Figure 47b the resultant bow vortex persists for the length of the ship and another vortical separation is apparent. Similar but less detailed results have been obtained with URANS simulations.

Despite the different configuration orientations of the air and sea facets, both are focused toward blunt leading-edge separation-induced vortical flows, and both bring advanced experimental and computational methods directed toward the development of enhanced understanding and prediction of these

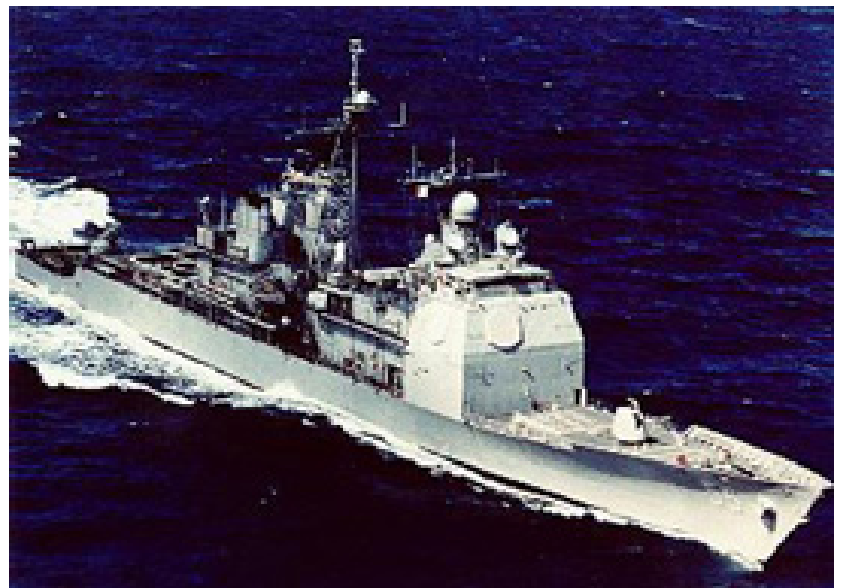

Figure 46. Naval surface combatant. flows. The RTO helps foster a very beneficial interaction between the air-oriented and sea-oriented scientific communities that otherwise tends to not occur.

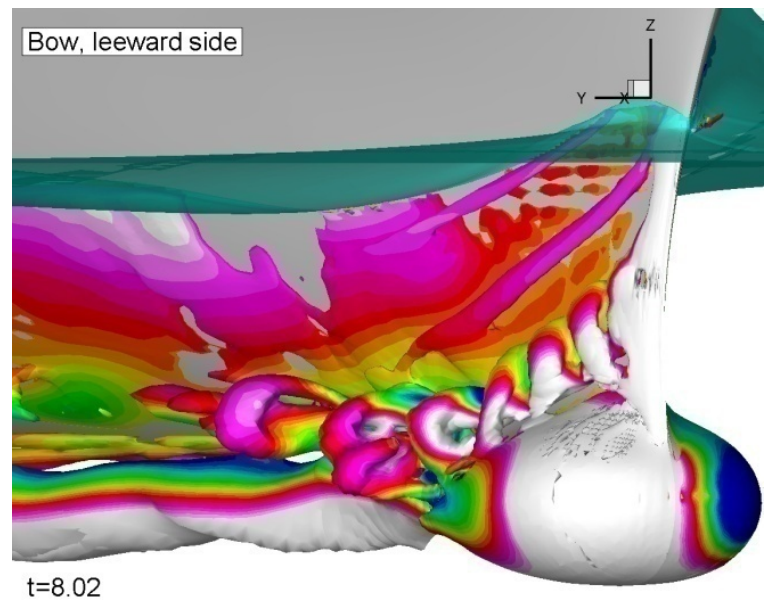

a) Bow

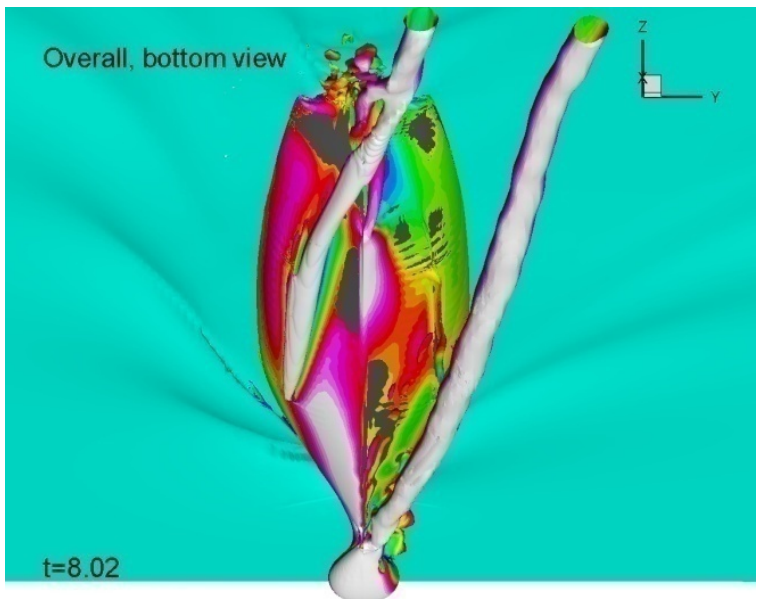

b) Stern view

Figure 47. Preliminary CFD simulation of bow separation. DES, Stern ${ }^{99}$ [2008].

\section{Concluding Remarks}

An overview of some factors affecting blunt leading-edge separation from swept and semi-slender wings has been presented. Selected examples were included to address both fundamental flow and configuration aerodynamic considerations. Despite the length of this article, it was this author's finding that considerably more information was available regarding this topic than space permitted, and only a subset of information could be included.

From a fundamental standpoint, the existence of various flow domains as regards classes of leading-edge separation, attachment-line transition, and wing leading-edge relaminarization were reviewed. The classifications chosen for this review have become very well established, and the domain boundaries demarcate different flow physics. It appears that a perspective toward the salient flow physics domains is very important for understanding blunt leading-edge separation effects for configuration aerodynamics. It also appears that basic airfoil separation characteristics carry over to low aspect ratio and swept wing aerodynamics more so than might be expected.

The importance of independent effects due to compressibility and Reynolds number as regards blunt leadingedge separation was also reviewed. An increase in Reynolds number delays blunt leading-edge separation whereas an increase in Mach number promotes the separation. This applies not only to airfoils, but also to the swept and semi-slender wings. Appropriate care must be exercised in interpreting data for which Mach and Reynolds numbers were varied simultaneously. 
From a configuration perspective, the onset and progression of blunt leading-edge separation continues to be a challenging problem. While progress has been demonstrated for both CFD simulations and experimental quantifications of this flow, there still remains a lack of understanding as to why some methods work better than others, or what the key underlying fluid parameters are. Part of this knowledge gap comes from the challenges associated with obtaining detailed measurements in the vicinity of blunt leading-edge separation for the wings of current interest.

One path forward comes from a marriage of relevant unit problem investigations with configuration aero/hydrodynamic research so as to develop enhanced understanding and improved prediction capability for these smoothsurface separated flows. Such campaigns require extensive computational and experimental resources, and multiorganizational entities such as TTCP and the RTO provide an enabling framework for these programs. They establish an opportunity among peers to design and execute focused experimental/computational campaigns to create knowledge for improving computational predictive capability of separated flow effects.

Finally the value of various focused or special sessions hosted by the AIAA must be noted. It is this author's observation that this is providing a very useful forum for integrated bodies of work from various sponsoring organizations to be captured as a collective for broad use by the aerospace community.

\section{References}

${ }^{1}$ Polhamus EC. Vortex Lift Research: Early Contributions and Some Current Challenges. NASA CP-2416, Vol 1, pp 1-30, Jul 1986.

${ }^{2}$ Whitcomb RT. A Study of the Zero-Lift Drag Characteristics of Wing-Body Combinations Near the Speed of Sound. NACA RM L52H08, Sep 1952.

${ }^{3}$ Abbott IA, and von Doenhoff AE. Theory of Wing Sections. Dover, 1959.

${ }^{4}$ Hummel D. On the Vortex Formation Over a Slender Wing at Large Incidence. AGARD CP-247, Paper 15, Jan 1979.

${ }^{5}$ Polhamus EC. A Concept of the Vortex Lift of Sharp-Edged Delta Wings Based on a Leading-Edge Suction Analogy. NASA TN D-3767, Dec 1966.

${ }^{6}$ Lamar JE. Extension of Leading-Edge-Suction Analogy to Wings with Separated Flow around the Side Edges at Subsonic Speeds. NASA TR-428, Oct 1974.

${ }^{7}$ Luckring JM. Reynolds Number, Compressibility, and Leading-Edge Bluntness Effects on Delta Wing Aerodynamics. ICAS ICAS04-414, Sep 2004.

${ }^{8}$ Wick BH. Chordwise and Spanwise Loadings Measured at Low Speed on a Triangular Wing Having an Aspect Ratio of Two and an NACA 0012 Airfoil Section. NACA TN 1650, Jun 1948.

${ }^{9}$ Gault DE. A Correlation of Low-Speed Airfoil Section Stalling Characteristics with Reynolds Number and Airfoil Geometry, NACA TN 3963, Mar 1957.

${ }^{10}$ Polhamus EC. A Survey of Reynolds Number and Wing Geometry Effects on Lift Characteristics in the Low Speed Stall Region. NASA CR-4745, Jun 1996.

${ }^{11}$ Haines AB. Scale effects on Weapon and Aircraft Aerodynamics. AGARD AG-323, Jul 1994.

${ }^{12}$ Loftin LK Jr, and Smith HA. Aerodynamic Characteristics of 15 NACA Airfoil Sections at Seven Reynolds Numbers from 0.7 x 106 to 9.0 x 106. NACA TN 1945, Oct 1949.

${ }^{13}$ Ladson CL. Effects of Independent Variation of Mach and Reynolds Numbers on the Low-Speed Aerodynamic Characteristics of the NACA 0012 Airfoil Section. NASA TM 4074, Oct 1988.

${ }^{14}$ Erlich E. Exemples de Recherches sur les Profils Dans la Soufflerie S 10 DU C.E.A.T. A Toulouse. ONERA TP 766.

${ }^{15}$ Multhopp H. Methods for Calculating the Lift Distribution of Wings (Subsonic Lifting-Surface Theory). ARC R\&M 2884, Jan. 1950.

${ }^{16}$ Lamar JE. A Modified Multhopp Approach for Predicting Lifting Pressures and Camber Shape for Composite Planforms in Subsonic Flow. NASA TN D-4427, Jul 1968

${ }^{17}$ Lan CE, and Lamar JE. On the Logarithmic-Singularity Correction in the Kernel-Function Method of Subsonic Lifting Surface Theory. NASA TN D-8513, Nov 1977.

${ }^{18}$ Poll DIA. Transition in the infinite swept attachment line boundary layer. Aeronautical Quarterly, Vol 30, pp 607-629, Nov 1979.

${ }^{19}$ Launder BE, and Jones WP. On the Prediction of Laminarization. ARC CP 1036, 1969.

${ }^{20}$ Treadgold DA, and Beasley JA. Some Examples of Methods for the Prediction of Boundary Layer Transition on Sheared Wings. AGARD R-602, Paper 2, Apr 1973.

${ }^{21}$ Ashill PR, Fulker JL, Simmons MJ, and Betts CJ. Flow Features of Highly Swept Wings at Subsonic and Supersonic Speeds. ICAS 90-3.9.1, Sep 1990.

${ }^{22}$ Ashill PR, and Betts CJ. A Study of the Flow around the Leading Edge of a Highly-Swept Wing in a Low-Speed Wind Tunnel. ASME Engineering Conference, Jun 1993.

${ }^{23}$ Henderson WP. Effects of Wing Leading-Edge Radius and Reynolds Number on Longitudinal Aerodynamic Characteristics of Highly Swept Wing-Body Configurations at Subsonic Speeds. NASA TN D-8361. Dec 1976.

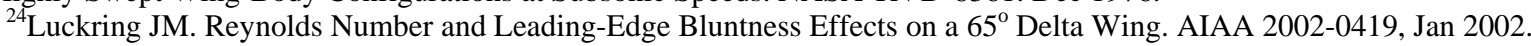


${ }^{25}$ Luckring JM. Transonic Reynolds Number and Leading-Edge Bluntness Effects on a $65^{\circ}$ Delta Wing. AIAA 2003-0753, Jan 2003.

${ }^{26}$ Luckring JM. Compressibility and Leading-Edge Bluntness Effects on a 65 Delta Wing. AIAA 2004-765, Jan 2004.

${ }^{27}$ Chu J, and Luckring JM. Experimental Surface Pressure Data Obtained on $65^{\circ}$ Delta Wing Across Reynolds Number and Mach Number Ranges. NASA TM-4645, Feb 1996.

${ }^{28}$ Billman GM, and Osbourne RA. High L/D extended range/payload fighter aircraft technology. AFRL-VA-WP-1999-3084, Nov 1998.

${ }^{29}$ Wong MD, McKenzie GJ, Ol MV, Petterson K, and Zhang S. Joint TTCP CFD Studies into the 1303 UCAV Performance: First Year Results. AIAA 2006-2984, Jun 2006.

${ }^{30}$ McParlin SC, Bruce RJ, Hepworth AG, and Rae AJ. Low speed wind tunnel tests on the 1303 UCAV Concept. AIAA 2006-2985, Jun 2006.

${ }^{31}$ Petterson K. Low-Speed Aerodynamic and Flowfield Characteristics of a UCAV. AIAA 2006-2986, Jun 2006.

${ }^{32}$ Wong MD, and Flores J. Application of OVERFLOW-MLP to the Analysis of the 1303 UCAV. AIAA 2006-2987, Jun 200633

${ }^{33}$ Milne ME, and Arthur MT. Evaluation of bespoke and commercial CFD methods for a UCAV configuration. AIAA 20062988, Jun 2006.

${ }^{34}$ Chung JJ, and Ghee TA. Numerical Investigation of UCAV 1303 Configuration with and without Simple Deployable Vortex Flaps. AIAA 2006-2989, Jun 2006.

${ }^{35}$ Ol MV. Water Tunnel Velocimetry Results for the 1303 UCAV Configuration. AIAA 2006-2990, Jun 2006.

${ }^{36}$ Woolvin SJ. A Conceptual Design Study of the 1303 UCAV Configuration. AIAA 2006-2991, Jun 2006.

${ }^{37}$ Bruce RJ, and Mundell ARG. Low speed wind tunnel tests on the 1303 UCAV concept. QINETIQ/FST/TR025502, QinetiQ Ltd., 2003.

${ }^{38}$ Bruce RJ. High speed wind tunnel tests on the 1303 UCAV concept. QINETIQ/FST/TR030214/1.0, QinetiQ Ltd., Jun 2003.

${ }^{39}$ Petterson K. CFD Analysis of the Low-Speed Aerodynamic Characteristics of a UCAV. AIAA 2006-1259, Jan 2006.

${ }^{40}$ Arthur MT, and Atkin CJ. Transition modeling for viscous flow prediction. AIAA 2006-3052, Jun 2006.

${ }^{41}$ Arthur MT, and Petterson K. A computational study of the low-speed flow over the 1303 UCAV configuration. AIAA 2007-4568, Jun 2007.

${ }^{42}$ Arthur MT, Horton HP, and Mughal MS. Modeling of Natural Transition in Properly Three-Dimensional Flows. AIAA 2009-3556, Jun 2009.

${ }^{43}$ RTO. Understanding and Modeling Vortical Flows to Improve the Technology readiness Level for Military Aircraft. RTOTR-AVT-113, Oct. 2009.

${ }^{44}$ Lamar JE, Obara CJ, Fisher BD, and Fisher DF. Flight, Wind-Tunnel, and a Computational Fluid Dynamics Comparison for Cranked Arrow Wing (F-16XL-1) at Subsonic and Transonic Speeds. NASA/TP-2001-210629, Feb 2001.

${ }^{45}$ Lamar JE. Cranked Arrow Wing (F-16XL-1) Flight Flow Physics with CFD Predictions at Subsonic and Transonic Speeds. RTO MP-069, Paper 44, Mar 2003.

${ }^{46}$ RTO. Advanced Flow Management. Part A - Vortex Flows and High Angle of Attack for Military Vehicles. RTO MP-069, Mar 2003.

${ }^{47}$ Boelens OJ, Spekreijse SP, Sytsma HA, and de Cock KMJ. Comparison of Measured and Simulated Flow Features for the Full-Scale F-16XL Aircraft. AIAA 2007-0489, Jan 2007.

${ }^{48}$ Badcock KJ. Evaluation of Results from a Reynolds Averaged Multiblock Code Against F-16XL Flight Data. AIAA 20070490, Jan 2007.

${ }^{49}$ Elmiligue AA, Abdol-Hamid KS, and Massey SJ. PAB3D Simulations for the CAWAPI F-16XL. AIAA 2007-0491, Jan 2007.

${ }^{50}$ Fritz W. Hybrid Grid RANS Solutions for the CAWAPI F-16XL. AIAA 2007-0492, Jan 2007.

${ }^{51}$ Morton SA, McDaniels DR, and Cummings RM. F-16XL Unsteady Simulations for the CAWAPI Facet of RTO Task Group AVT-113. AIAA 2007-0493, Jan 2007.

${ }^{52}$ Görtz S. and Jirásek A. Unstructured Steady/Unsteady Solutions with Edge for CAWAPI F-16XL at KTH/FOI. AIAA 2007-0678, Jan 2007.

${ }^{53}$ Michal T, Oser M, Mani M, and Roos F. BCFD Unstructured-Grid Predictions on the F-16XL (CAWAPI) Aircraft. AIAA 2007-0679, Jan 2007.

${ }^{54}$ Davis MB, Reed C, and Yagle P. Hybrid Grid Solutions on the (CAWAPI) F-16XL Using Falcon v4. AIAA 2007-0680, Jan 2007.

${ }^{55}$ Karman S, Mitchell B. and Sawyer, S. Unstructured Grid Solutions of CAWAPI F-16XL by UT SimCenter. AIAA 20070681, Jan 2007.

${ }^{56}$ Lamar JE, and Abdol-Hamid K. USM3D Unstructured Grid Solutions for CAWAPI at NASA LaRC. AIAA 2007-0682, Jan 2007.

${ }^{57}$ Rizzi A, Badcock KJ, Jirásek A, and Boelens OJ. What was Learned from Numerical Simulations of F-16XL (CAWAPI) at Flight Conditions. AIAA 2007-0683, Jan 2007.

${ }^{58}$ Luckring JM. An Overview of National Transonic Facility Investigations for High Performance Military Aerodynamics. AIAA 2001-906, Jan 2001. 
Chicago, IL

${ }^{59}$ Hummel D, and Redeker G. A New Vortex Flow Experiment for Computer Code Validation. RTO MP-069, Paper 9, Mar 2003.

${ }^{60}$ Hummel D. Review of the Second International Vortex Flow Experiment (VFE-2). AIAA 2008-377, Jan 2008.

${ }^{61}$ Luckring JM. Initial Experiments and Analysis of Blunt-Edge Vortex Flows. AIAA 2008-378, Jan 2008.

${ }^{62}$ Konrath R, Klein C, and Schroeder A. PSP and PIV Investigations on the VFE-2 Configuration in Sub- and Transonic Flow. AIAA 2008-379, Jan 2008

${ }^{63}$ LeRoy JF, Rodriguez O, and Kurun S. Experimental and CFD Contribution to Delta Wing Vortical Flow Understanding. AIAA 2008-0380, Jan 2008.

${ }^{64}$ Furman A, and Breitsamter Ch, Turbulent and Unsteady Flow Characteristics of Delta Wing Vortex Systems. AIAA 20080381, Jan 2008.

${ }^{65}$ Coton F, Mat S, and Galbraith R. Low Speed Wind Tunnel Characterization of the VFE-2 Wing. AIAA 2008-0382, Jan 2008.

${ }^{66}$ Luckring JM, and Hummel D. What Was Learned from the New VFE-2 Experiments. AIAA 2008-383, Jan 2008.

${ }^{67}$ Nangia RK. Semi-Empirical Prediction of Vortex Onset \& Progression on 65 Delta Wings (RTO-AVT-113, VFE-2 Facet). AIAA 2008-384, Jan 2008

${ }^{68}$ Fritz W. Numerical Simulation of the Peculiar Subsonic Flow-Field about the VFE-2 Delta Wing with Rounded Leading Edge. AIAA 2008-393, Jan 2008.

${ }^{69}$ Gürdamar E, Ortakaya Y, Kaya S, and Korkem, B. Some Factors Influencing the Vortical Flow Structures on Delta Wings. AIAA 2008-394, Jan 2008.

${ }^{70}$ Shiavetta LA, Boelens OJ, Krippa S, Fritz W, and Badcock KJ. Shock Effects on Delta Wing Vortex Breakdown. AIAA 2008-395, Jan 2008.

${ }^{71}$ Cummings RM, and Schütte A. Detached-Eddy Simulation of the Vortical Flowfield about the VFE-2 Delta Wing. AIAA 2008-396, Jan 2008.

${ }^{72}$ Crippa S, and Rizzi, A. Steady, Subsonic CFD Analysis of the VFE-2 Configuration and Comparison to Wind Tunnel Data. AIAA 2008-397, Jan 2008.

${ }^{73}$ Schütte A, and Lüdeke H. Numerical investigations on the VFE-2 65-Degree rounded leading edge delta wing using the unstructured DLR-TAU-Code. AIAA 2008-398, Jan 2008

${ }^{74}$ Fritz W. What Was Learned From the Numerical Simulations for the VFE-2. AIAA 2008-399, Jan 2008.

${ }^{75}$ Hummel D. Effects of Boundary Layer Formation on the Vortical Flow above Slender Delta Wing. RTO MP-AVT-111, Paper 30, Oct 2004.

${ }^{76}$ Konrath R, Schröder A, and Kompenhans J. Analysis of PIV Results Obtained for the VFE-2 $65^{\circ}$ Delta Wing Configuration at Sub- and Transonic Speeds. AIAA 2006-3003, Jun 2006.

${ }^{77}$ Cummings R, and Schütte A. An Integrated Computational/Experimental Approach to UCAV Stability \& Control Estimation: Overview of NATO RTO AVT- 161. AIAA 2010-4392, Jun 2010.

${ }^{78}$ Loeser T, Vicroy D, and Schütte A. SACCON Static Wind Tunnel Tests at DNW- NWB and 14'x22' NASA LaRC. AIAA 2010-4393, Jun 2010.

${ }^{79}$ Vicroy D, and Loeser T. SACCON Dynamic Wind Tunnel Tests at DNW- NWB and 14'x22' NASA LaRC. AIAA 20104394, Jun 2010.

${ }^{80}$ Gilliot A. Static and Dynamic SACCON PIV Tests - Part I: Forward Flowfield. AIAA 2010-4395, Jun 2010.

${ }^{81}$ Konrath R, Roosenboom E, Schröder A, Pallek D, and Otter D. Static and Dynamic SACCON PIV Tests - Part II: Aft Flow Field. AIAA 2010-4396, Jun 2010.

${ }^{82}$ Frink NT. Strategy for Dynamic CFD Simulations on SACCON Configuration. AIAA 2010-4559, Jun 2010.

${ }^{83}$ Vallespin D, Boelens O, and Badcock K. SACCON CFD Simulations Using Structured Grid Approaches. AIAA 20104560, Jun 2010.

${ }^{84}$ Tormalm M, Tomac M, and Schmidt S. Computational Study of Static And Dynamic Vortical Flow over the Delta Wing SACCON Configuration Using the FOI Flow Solver Edge. AIAA 2010-4561, Jun 2010.

${ }^{85}$ Le Roy J. SACCON CFD Static and Dynamic Derivatives Using elsA. AIAA 2010-4562, Jun 2010.

${ }^{86}$ Chakravarthy S, and Chi D. SACCON CFD Simulations Using CFD++. AIAA 2010-4563, Jun 2010.

${ }^{870}$ Schütte A, Hummel D, and Hitzel S. Numerical and Experimental Analyses of the Vortical Flow Around the SACCON Configuration. AIAA 2010-4690, Jun 2010.

${ }^{88}$ Cummings R, Petterson K, Jirasek A, and Schmidt S. SACCON Static and Dynamic Motion Flow Physics Simulations Using COBALT. AIAA 2010-4691, Jun 2010.

${ }^{89}$ Thompson JR, Frink NT, and Murphy PC. Guidelines for Computing Longitudinal Dynamic Derivatives on Subsonic Transport. AIAA 2010-4819, Jun 2010.

${ }^{90}$ Boelens OJ. CFD Analysis of the Flow Around the X-31 Aircraft at High Angle of Attack. AIAA 2009-3628, Jun 2009.

${ }^{91}$ Oberkampf WL, and Trucano TG. Verification and Validation in Computational Fluid Dynamics. Progress in Aerospace Sciences, Vol 38, 2002.

${ }^{92}$ AIAA. Guide for the Verification and Validation of Computational Fluid Dynamics Simulations. AIAA G-077-1998, Jan 1998.

${ }^{93}$ ASME. Guide for Verification and Validation in Computational Solid Mechanics. ASME V\&V 10-2006, Dec 2006.

${ }^{94}$ ASME. Standard for Verification and Validation in Computational Fluid Dynamics and Heat Transfer. ASME V\&V 202009, Nov 2009. 
${ }^{95}$ Mirande J, Schmitt V, and Werle H. Vortex Pattern Developing on the Upper Surface of a Swept Wing at High Angle of Attack. AGARD CP 247, Paper 12, Jan 1979.

${ }^{96}$ Poll DIA. On the Generation and Subsequent Development of Spiral Vortex Flow Over a Swept-Back Wing. AGARD CP342, Paper 6, Jul 1983.

${ }^{97}$ Stern F, Agdrup K, Kim SY, Hochbaum AC, Rhee KP, Quadvlieg F, Perdon P, Hino T, Broglia R, and Gorski J. Lessons learnt from the Workshop on V\&V of ship maneuvering simulation methods: SIMMAN 2009. MARSIM'09, Aug 2009.

${ }^{98}$ Stern F, Bhushan S, Carrica P, and Yang J. Large scale parallel computing and scalability study for static DTMB 5415 at 20 deg drift using CFDShip-Iowa V.4 and Straight ahead using CFDShip-Iowa V.6. 21st International Conference Parallel CFD, Parallel CFD 2009, May 2009.

${ }^{99}$ Stern F. Private communication. Oct 2008. 